\title{
ASYMPTOTIC COMPLETENESS FOR CLASSES OF TWO, THREE, AND FOUR PARTICLE SCHRÖDINGER OPERATORS ${ }^{1}$ \\ BY
}

\author{
GEORGE A. HAGEDORN ${ }^{2}$
}

\begin{abstract}
Formulas for the resolvent $(z-H)^{-1}$ are derived, where $H=H_{0}+$ $\Sigma_{i<j} \lambda_{i j} V_{i j}$ is an $N$ particle Schrödinger operator with the center of mass motion removed. For a large class of two-body potentials and generic couplings $\left\{\lambda_{i j}\right\}$, these formulas are used to prove asymptotic completeness in the $N<4$ body problem when the space dimension is $m>3$. The allowed potentials belong to a space of dilation analytic multiplication operators which fall off more rapidly than $r^{-2-\varepsilon}$ at $\infty$. In particular, Yukawa potentials, generalized Yukawa potentials, and potentials of the form $(1+r)^{-2-e}$ are allowed.
\end{abstract}

I. Introduction. The scattering theory of two particle nonrelativistic quantum mechanics is reasonably well understood for short range potentials. The wave operators are asymptotically complete, and the singular continuous spectrum is empty (see Agmon [1], Enss [42], and Reed and Simon [24]). Much less is known about general $N$ particle scattering.

For $N=3$ and space dimension $m \geqslant 3$, Faddeev [6] first proved completeness of the wave operators for a large class of potentials and almost all coupling constants. These results have been simplified and extended by Ginibre and Moulin [7], Thomas [36], and Howland [11]. All of these authors require the potentials to fall off faster than $r^{-2-\varepsilon}$ at infinity and obtain Kuroda completeness for almost all couplings. Mourre [19] has extended these results to allow potentials which fall faster than $r^{-1-\varepsilon}$, as long as the potentials are repulsive for large $r$.

For general $N$, Balslev and Combes [3] have proved the absence of singular continuous spectrum for dilation analytic potentials. Asymptotic completeness for potentials falling off faster than $r^{-2-\varepsilon}$ has been proved for small couplings by Iorio and O'Carrol [13], and for repulsive potentials falling off faster than $r^{-5 / 2-\varepsilon}$ by Lavine [18]. (These are single channel results only.) Hepp [10] and Sigal [26] have reduced general $\mathrm{N}$-body asymptotic completeness to the verification of properties of certain operators. They conjecture that these hold for generic potentials in certain classes.

Simultaneous to our announcement [9] of results, Sigal [28] announced a proof of asymptotic completeness for generic elements of a space of dilation analytic potentials and almost all couplings. More recently, van Winter [43] announced a

Received by the editors May 23, 1978.

AMS (MOS) subject classifications (1970). Primary 47A40, 81A48.

${ }^{1}$ Based on a Princeton University Doctoral Thesis.

${ }^{2}$ Partially supported by an NSF Graduate Fellowship. 
proof of completeness for some analytic Hilbert-Schmidt potentials. Also, Enss [42] has obtained some many body results by beautiful, time dependent methods.

Our main results are the following:

THEOREM I.1. Let $m \geqslant 3$ and $N \leqslant 4$. Let

$$
\tilde{H}=-\sum_{i=1}^{N}\left(2 m_{i}\right)^{-1} \Delta_{i}+\sum_{i<j} \lambda_{i j} V_{i j} \text { on } L^{2}\left(\mathbf{R}^{N m}\right)
$$

be the Schrödinger operator for $N$ particles moving in $m$ dimensions. Let $H=H_{0}+$ $\Sigma_{i<j} \lambda_{i j} V_{i j}$ denote the Schrödinger operator on $L^{2}\left(\mathbf{R}^{(N-1) m}\right)$ for the same system with the center of mass motion removed. Assume each $V_{i j}$ may be factored as $V_{i j}=U_{i j} W_{i j}$ so that

(i) each $U_{i j}$ and $W_{i j}$ is dilation analytic in some strip,

(ii) $\left(1+x_{i j}^{2}\right)^{\gamma} U_{i j}\left(x_{i j}\right)$ and $\left(1+x_{i j}^{2}\right)^{\gamma} W_{i j}$ belong to $L^{p}\left(R^{m}\right)+L^{\infty}\left(R^{m}\right)$ for some $p>m$ and $\gamma>\frac{1}{2}$,

(iii) bound state energies of three body subsystems are nonpositive when $N=4$.

Then for generic couplings $\left\{\lambda_{i j}\right\}$, asymptotic completeness holds.

THEOREM I.2. The set of generic couplings for Theorem I.1 is large in the sense that its complement is a closed set of Lebesgue measure zero.

Remarks. (1) Hypothesis (ii) of Theorem I.1 implies that $U_{i j}\left(-\Delta_{i j}+1\right)^{-1 / 2}$ and $W_{i j}\left(-\Delta_{i j}+1\right)^{-1 / 2}$ are compact on $L^{2}\left(R^{m}\right)$. Thus, $H=H_{0}+\Sigma \lambda_{i j} V_{i j}$ is understood in the sense of quadratic forms.

(2) Balslev [2] and Simon [32] have given sufficient conditions for Hypothesis (iii) to hold. See Theorem II.10. Yukawa potentials, generalized Yukawa potentials, and potentials of the form $(1+r)^{-2-\varepsilon}$ obey these conditions. Therefore, for these potentials, Theorems I.1 and I.2 establish asymptotic completeness for generic couplings.

(3) For the generic couplings, $H$ has finitely many thresholds. Sigal [27] has also obtained this result.

(4) The generic couplings are precisely those for which no cluster Hamiltonian has a threshold resonance or threshold bound state.

(5) Theorem I.1 will be proved under the assumption that all the masses are finite. If one of the masses is infinite, the result still holds, but several lemmas from $\S \mathrm{V}$ must be modified. Various $\rho$ functions must be changed, and in many instances, Case 2 of Lemma II.3 must be used in place of Case 1.

Our proof of Theorems I.1 and I.2 is organized as follows: In $\S \mathrm{II}$, we collect various technical results from the literature. Little is new here, except that weighted spaces $L_{\delta}^{2}$ are replaced by more general $L_{\delta}^{p}$ spaces. In $\S$ III, $N$-body Kuroda completeness is reduced to the study of the resolvent $(z-H)^{-1}$. Formulas for the $N$-body resolvent are derived in §IV. Equation (IV.4) is a generalization of Faddeev's three body formula [6] to the $N$-body case. It is much simpler than the Yakubovskii formula [41], but we have not been able to prove that it has all the desirable properties of the Yakubovskii equation. A second, more complicated 
formula (equation (IV.5)), is derived from equation (IV.4). This formula is equivalent to a formula of Narodetskii and Yakubovskii [20]. Equation (IV.5) is modified for $N \leqslant 4$, in order to study scattering. In $\S \mathrm{V}$, these equations are used to prove properties of $(z-H)^{-1}$ for $N<4$ under certain hypotheses. The validity of these hypotheses is studied in $\S \mathrm{VI}$.

Given the absence of singular continuous spectrum (Theorem II.8) and the existence of wave operators (Theorem II.12), Theorems I.1 and I.2 follow immediately from Theorem III.1, Propositions V.1, 2, and 3, and Proposition VI.3.

REMARK. The principal reason we do not have results for $N>5$ is that we have been unable to prove the absence of spurious zeros in equation (IV.10).

ACKNOWLEDGements. I am very grateful to Professor Barry Simon for introducing me to many body Schrödinger operators and for his advice throughout this work. In addition, I would like to thank the ETH and IHES for their hospitality during the Spring and Summer of 1977, and Dr. I. M. Sigal for pointing out an error in a rough draft.

Preliminary definitions. The Schrödinger operator for a system of $N$ particles moving in $m$ dimensions is $\tilde{H}=-\sum_{i=1}^{N}\left(2 m_{i}\right)^{-1} \Delta_{i}+\sum_{i<j} V_{i j}$ on $L^{2}\left(\mathbf{R}^{N m}\right)$. The mass of the $i$ th particle is $m_{i}, \Delta_{i}$ is the Laplacian in the $i$ th variable, and the potential energy between particles $i$ and $j$ is the multiplication operator $V_{i j}\left(r_{i}-r_{j}\right)$. We remove the trivial center of mass motion (see [23]) from $\tilde{H}$, to obtain $H=H_{0}+$ $\Sigma_{i<j} V_{i j}$ on $\mathcal{H}=L^{2}\left(\mathbf{R}^{(N-1) m}\right)$.

A cluster decomposition $D=\left\{C_{i}\right\}_{i=1}^{k}$ is a partition of the set $\{1,2, \ldots, N\}$ into $k$ disjoint clusters $C_{i}$. The Hamiltonian $H_{D}$ is defined as $H_{0}+V_{D}$, where $V_{D}$ is the sum of all $V_{i j}$ with $i$ and $j$ in the same cluster. The Hilbert space $\mathcal{H}$ may be decomposed as $\mathcal{K}_{1} \otimes \mathcal{F}_{2} \otimes \cdots \otimes \mathcal{K}_{k} \otimes \mathcal{H}(D)$, so that

$$
\begin{aligned}
H_{D}= & h_{1} \otimes 1 \otimes \cdots \otimes 1+1 \otimes h_{2} \otimes \cdots \otimes 1+\cdots \\
& +1 \otimes \cdots \otimes h_{k} \otimes 1+1 \otimes \cdots \otimes 1 \otimes K_{D} .
\end{aligned}
$$

The Hamiltonian $h_{i}$ corresponds to the energy of the particles in cluster $C_{i}$ alone. $K_{D}$ is the kinetic energy of the centers of mass of the clusters in $D$.

For each cluster Hamiltonian $h_{i}$, we choose eigenfunctions $\eta_{j}^{(i)}$ of $h_{i}$, so that $\left\{\eta_{j}^{(i)}\right\}$ is an orthonormal basis for the subspace of $\mathcal{H}_{i}$ generated by the eigenfunctions of $h_{i}$.

A channel $\alpha$ is a cluster decomposition $D(\alpha)$, together with an eigenfunction $\eta^{(i)} \in\left\{\eta_{j}^{(i)}\right\}$ for each $h_{i}$. We define $E_{\alpha}=\sum_{i=1}^{k} E_{i}$, where $h_{i} \eta^{(i)}=E_{i} \eta^{(i)}$, and let $P_{\alpha}$ : $\mathcal{H} \rightarrow \mathcal{H}$ denote the orthogonal projection onto all vectors of the form $\eta^{(1)} \otimes \eta^{(2)}$ $\otimes \cdots \otimes \eta^{(k)} \otimes \phi$, where $\phi \in \mathcal{H}(D(\alpha))$ is arbitrary. Let $T_{\alpha}=1 \otimes 1 \otimes \cdots \otimes 1 \otimes$ $K_{D(\alpha)}+E_{\alpha}$, so that $H_{D(\alpha)} P_{\alpha}=T_{\alpha} P_{\alpha}$.

The channel wave operators are defined as

$$
\Omega_{\alpha}^{\mp}=\text { strong- } \operatorname{limit}_{t \rightarrow \pm \infty} e^{i t H} e^{-i t T_{\alpha} P_{\alpha}}
$$

if the limits exist. The wave operators are asymptotically complete if

$$
\mathcal{H}=\bigoplus_{\alpha} \operatorname{Ran} \Omega_{\alpha}^{+} \oplus \mathcal{H} \text { bound }=\bigoplus_{\alpha} \operatorname{Ran} \Omega_{\alpha}^{-} \oplus \mathcal{H}_{\text {bound }},
$$

where $\mathcal{F}_{\text {bound }}$ is the span of the eigenfunctions for $H$. 
Each $\Omega_{\alpha}^{ \pm}$is an isometry, so the projection onto $\operatorname{Ran} \Omega_{\alpha}^{ \pm}$is $\Omega_{\alpha}^{ \pm} \Omega_{\alpha}^{ \pm *}$. Since the channel wave operators have orthogonal ranges, the projection onto $\bigoplus_{\alpha} \operatorname{Ran} \Omega_{\alpha}^{ \pm}$is $\Sigma_{\alpha} \Omega_{\alpha}^{ \pm} \Omega_{\alpha}^{ \pm *}$.

The ranges of the wave operators are contained in $\mathcal{H}_{\text {a.c. }}$, the absolutely continuous subspace for $H$. Thus, $\bigoplus \operatorname{Ran} \Omega_{\alpha}^{ \pm} \subseteq \mathcal{H}_{\text {a.c. }}$. A weaker form of completeness is the condition $P_{\text {a.c. }}=\Sigma_{\alpha} \Omega_{\alpha}^{ \pm} \Omega_{\alpha}^{ \pm *}$. This is called Kuroda completeness. Kuroda completeness implies asymptotic completeness if $H$ has no singular continuous spectrum.

II. Technical devices. In this section we collect several results which are required for the other sections.

A. Jacobi coordinates (see Reed and Simon [23]).

We will use two types of coordinate systems for $\mathbf{R}^{(N-1) m}$. Both of these systems have the property that $H_{0}$ is of the form $-\sum_{i=1}^{N-1}\left(2 \mu_{i}\right)^{-1} \Delta_{i}$, where $\mu_{i}>0$ and $\Delta_{i}$ is the Laplacian in the $i$ th coordinate. The particle positions $r_{i}$ and their differences $r_{i}-r_{j}$ are not simple in these coordinates.

To obtain Jacobi coordinates $\left\{\zeta_{i}\right\}$, we begin by labelling the particles by the numbers $1,2, \ldots, N$. Then, for $1 \leqslant j \leqslant N-1$, we let $\zeta_{j}$ denote the vector from the center of mass particles $1,2, \ldots, j$ to particle $j+1$. Explicitly, $\zeta_{j}=r_{j+1}-$ $\left(\Sigma_{i<j} m_{i}\right)^{-1} \Sigma_{i<j} m_{i} r_{i}$, where $r_{l}$ denotes the position of particle $l$.

The second type of coordinate system is used to put $K_{D}$, the free Hamiltonian for the centers of mass of the clusters of $D$, into the form $-\sum_{i=1}^{k-1}\left(2 \mu_{i}\right)^{-1} \Delta^{\left(\zeta_{i}\right)}$. For each cluster $C_{i}$, we choose Jacobi coordinates $\xi_{1}^{i}, \xi_{2}^{i}, \ldots, \xi_{n(i)-1}^{i}$ for the $n(i)$ particles labelled by the elements of $C_{i}$. Then we choose Jacobi coordinates $\zeta_{1}, \zeta_{2}, \ldots, \zeta_{k-1}$ for the centers of mass of the clusters. The resulting coordinates

$$
\xi_{1}^{1}, \ldots, \xi_{n(1)-1}^{1}, \quad \xi_{1}^{2}, \ldots, \xi_{n(k)-1}^{k}, \quad \zeta_{1}, \ldots, \zeta_{k-1}
$$

are called clustered Jacobi coordinates for the cluster decomposition $D$.

B. Quadratic form techniques.

Quadratic forms are used to define the Hamiltonian $H$ and to make sense out of various expressions involving resolvents, potentials, and square roots of the potentials.

The free Hamiltonian $H_{0}$ is a positive selfadjoint operator on $\mathcal{H}$. We let $\mathcal{H}_{+1}$ denote the operator domain of $H_{0}^{1 / 2}$ with inner product $\langle\phi, \psi\rangle_{+1}=$ $\langle\phi, \psi\rangle+\left\langle H_{0}^{1 / 2} \phi, H_{0}^{1 / 2} \psi\right\rangle$. Since $\mathcal{H}_{+1} \subseteq \mathcal{H}$, each $\phi \in \mathcal{H}$ defines a bounded linear functional on $\mathcal{H}_{+1}$ by $\psi \rightarrow\langle\phi, \psi\rangle$. Thus, $\mathcal{H}$ may be viewed as a subspace of the dual space $\mathcal{H}_{-1}$ of $\mathcal{H}_{+1}$.

For each pair $i, j, L^{2}\left(\mathbf{R}^{(N-1) m}\right)$ decomposes into $L^{2}\left(\mathbf{R}^{m}\right) \otimes L^{2}\left(\mathbf{R}^{(N-2) m}\right)$, where the first factor denotes functions of $x_{i j}$. Under this decomposition, $V_{i j}=v_{i j} \otimes 1$. We assume $v_{i j}=u_{i j} w_{i j}$ such that $u_{i j}\left(-\Delta_{i j}+1\right)^{-1 / 2}$ and $w_{i j}\left(-\Delta_{i j}+1\right)^{-1 / 2}$ are compact on $L^{2}\left(\mathbf{R}^{m}\right)$.

We denote $u_{i j} \otimes 1$ by $U_{i j}$ and $w_{i j} \otimes 1$ by $W_{i j}$. The assumptions on $u_{i j}$ and $w_{i j}$ imply that $U_{i j}: \mathcal{H} \rightarrow \mathcal{H}_{-1}$ and $W_{i j}: \mathcal{H}_{+1} \rightarrow \mathcal{H}$ are bounded. Moreover [22], for each $\varepsilon>0$, there exists $a>0$, such that 


$$
\left|\left\langle\psi, V_{i j} \psi\right\rangle\right|=\left|\left\langle U_{i j}^{*} \psi, W_{i j} \psi\right\rangle\right|\left\langle\varepsilon\|\psi\|_{+1}^{2}+a\|\psi\|^{2}\right.
$$

for all $\psi \in \mathcal{K}_{+1}$.

Given this bound, the $K L M N$ Theorem [22] implies that there is a unique selfadjoint operator $H$ with $D(H) \subseteq \mathcal{H}_{+1}$, such that

$$
\langle\psi, H \psi\rangle=\left\langle\psi,\left(H_{0}+\sum_{i<j} V_{i j}\right) \psi\right\rangle
$$

for $\psi \in \mathcal{K}_{+1}$. For each cluster decomposition $D$, the Hamiltonian $H_{D}$ is defined by the same method.

If $z \notin \sigma\left(H_{D}\right)$, then $\left(z-H_{D}\right)^{-1}$ is bounded from $\mathcal{K}_{-1}$ into $\mathcal{K}_{+1}$. Hence, products of the form $W_{i j}\left(z-H_{D}\right)^{-1} U_{k l}$ are bounded on $\mathcal{H}$.

C. Estimates related to Kato smoothness.

Although Kato smoothness [15] does not play a central role in our asymptotic completeness proof, some estimates from the theory of smooth perturbations will be used.

At least seven equivalent definitions of Kato smoothness are available (see [15], [24]). For our purposes, the following one is the most convenient.

Definition. A closed operator $F$ is called Kato smooth with respect to a selfadjoint operator $A$, if

$$
\|F\|_{A}^{2}=(2 \pi)^{-1} \sup _{\substack{z \notin \mathbf{R}, \phi \in D\left(F^{*}\right) \\\|\phi\|=1}}\left|\left\langle F^{*} \phi,\left[(z-A)^{-1}-(\bar{z}-A)^{-1}\right] F^{*} \phi\right\rangle\right|<\infty .
$$

Lemma II.1. Let $\phi_{1}: \mathbf{R} \rightarrow \mathcal{H}$ and $\phi_{2}: \mathbf{R} \rightarrow \mathcal{H}$ belong to $L^{2}(\mathbf{R}, \mathcal{H C})$, where $\mathcal{H}$ is a Hilbert space; if

$$
\hat{\phi}_{j}(\lambda)=(2 \pi)^{-1 / 2} \text { l.i.m. } \int_{\mathbf{R}} \phi_{j}(t) e^{-i t \lambda} d t \text { for } j=1,2,
$$

then

$$
\int_{\mathbf{R}}\left\langle\phi_{1}(t), \phi_{2}(t)\right\rangle d t=\int_{\mathbf{R}}\left\langle\hat{\phi}_{1}(\lambda), \hat{\phi}_{2}(\lambda)\right\rangle d \lambda .
$$

Proof. The usual Plancherel theorem shows that Fourier transform ${ }^{\wedge}: L^{2}(\mathbf{R}) \rightarrow$ $L^{2}(\mathbf{R})$ is unitary. Consequently, ${ }^{\wedge} \otimes 1: L^{2}(\mathbf{R}) \otimes \mathcal{H} \rightarrow L^{2}(\mathbf{R}) \otimes \mathscr{H}$ is unitary. The lemma follows from this by the natural identification of $L^{2}(\mathbf{R}) \otimes \mathcal{H}$ with $L^{2}(\mathbf{R}, \mathcal{H})$.

REMARK. In the following lemma we prove an inequality. Equality can be proved [15], [24], but we will only use the inequality.

Lema II.2. Suppose F is Kato smooth with respect to A. Then

$$
\|F\|_{A}^{2}=\pi^{-1} \sup _{\substack{z \notin \mathbb{R}, \phi \in D\left(F^{*}\right) \\\|\phi\|=1}}|\operatorname{Im} z|\left\|(z-A)^{-1} F^{*} \phi\right\|^{2}
$$

and

$$
\|F\|_{A}^{2}>(2 \pi)^{-1} \sup _{\|\phi\|=1} \int_{-\infty}^{\infty}\left\|F e^{-i t A} \phi\right\|^{2} d t .
$$


Proof.

$$
\begin{aligned}
(2 \pi)^{-1} \mid\left\langle F^{*} \phi,\right. & {\left.\left[(z-A)^{-1}-(\bar{z}-A)^{-1}\right] F^{*} \phi\right\rangle \mid } \\
= & (2 \pi)^{-1}|\bar{z}-z|\left|\left\langle F^{*} \phi,(\bar{z}-A)^{-1}(z-A)^{-1} F^{*} \phi\right\rangle\right| \\
& =\pi^{-1}|\operatorname{Im} z|\left\|(z-A)^{-1} F^{*} \phi\right\|^{2} .
\end{aligned}
$$

The first statement of the lemma follows by taking the supremum over $z \notin \mathbf{R}$ and $\phi \in D\left(F^{*}\right)$ with $\|\phi\|=1$.

The operator

$$
(2 \pi i)^{-1}\left[(\bar{z}-A)^{-1}-(z-A)^{-1}\right]=\pi^{-1}(\operatorname{Im} z)(z-A)^{-1}(\bar{z}-A)^{-1}
$$

is positive when $\operatorname{Im} z>0$. Let $K(z)$ be its positive square root. Since $F$ is $A$-smooth, $\left\|K(z) F^{*} \phi\right\|^{2} \leqslant\|F\|_{A}^{2}\|\phi\|^{2}$ for all $\phi \in D\left(F^{*}\right)$. Therefore, $\operatorname{Ran} K(z) \subseteq$ $D(F)$ and $\|F K(z)\|<\|F\|_{A}$.

If $\phi(\lambda)=\left[(\lambda+i \varepsilon-A)^{-1}-(\lambda-i \varepsilon-A)^{-1}\right] \phi$, then explicit computation shows $\hat{\phi}(t)=i e^{-\varepsilon|t|} e^{-i t A} \phi$. Thus, by Lemma II.1,

$$
\begin{aligned}
(2 \pi)^{-1} & \int_{-\infty}^{\infty}\left\|F e^{-i t A} \phi\right\|^{2} e^{-2 \varepsilon|t|} d t \\
& =(2 \pi)^{-2} \int_{-\infty}^{\infty}\left\|F\left[(\lambda+i \varepsilon-A)^{-1}-(\lambda-i \varepsilon-A)^{-1}\right] \phi\right\|^{2} d \lambda \\
& <\int_{-\infty}^{\infty}\|F K(\lambda+i \varepsilon)\|^{2}\|K(\lambda+i \varepsilon) \phi\|^{2} d \lambda \\
& <\|F\|_{A}^{2} \int_{-\infty}^{\infty}\|K(\lambda+i \varepsilon) \phi\|^{2} d \lambda \\
& =(2 \pi i)^{-1}\|F\|_{A}^{2} \int_{-\infty}^{\infty}\left\langle\phi,\left[(\lambda-i \varepsilon-A)^{-1}-(\lambda+i \varepsilon-A)^{-1}\right] \phi\right\rangle d \lambda \\
& =\|F\|_{A}^{2} \frac{\varepsilon}{\pi} \int_{-\infty}^{\infty} \int_{-\infty}^{\infty} \frac{1}{(\lambda-\mu)^{2}+\varepsilon^{2}} d\langle\phi, E(\mu) \phi\rangle d \lambda \\
& =\|F\|_{A}^{2}\|\phi\|^{2} .
\end{aligned}
$$

The lemma follows by taking $\varepsilon$ to zero.

Definition. For $1 \leqslant p \leqslant \infty$ and $\delta \in \mathbf{R}, L_{\delta}^{P}\left(\mathbf{R}^{m}\right)$ is the Banach space of functions $f: \mathbf{R}^{m} \rightarrow \mathbf{C}$, such that $\left(1+x^{2}\right)^{\delta / 2} f(x) \in L^{P}\left(\mathbf{R}^{m}\right)$. The $L_{\delta}^{P}$-norm of $f$ is the $L^{P}$-norm of $\left(1+x^{2}\right)^{\delta / 2} f(x)$.

REMARKs. (1) Lemma II.3 extends results of Iorio and O'Carrol [13].

(2) If the operators $F_{1}$ and $F_{2}$ are equal in Lemma II.3, then we conclude that $F_{1}^{*}=F_{2}^{*}$ is $H_{D}$-smooth.

LEMMA II.3. Let $D$ be a cluster decomposition with at least two clusters. Let $\delta>1$, $m>3, p_{1}>m$, and $p_{2}>m$. Suppose $\left\{\zeta_{1}, \ldots, \zeta_{k-1}\right\}$ and $\left\{\xi_{1}, \ldots, \xi_{k-1}\right\}$ are two (possibly identical) choices for the intercluster clustered Jacobi coordinates corresponding to the cluster decomposition $D$. Suppose $F_{1} \in L_{\delta}^{P_{1}}\left(\mathbf{R}^{m}\right)$ and $F_{2} \in L_{\delta}^{P_{2}}\left(\mathbf{R}^{m}\right)$. 
Let $F_{1}$ and $F_{2}$ denote multiplication by $F_{1}\left(\zeta_{1}\right)$ and $F_{2}\left(\xi_{1}\right)$, respectively. Since $\left\{\zeta_{i}\right\}$ and $\left\{\xi_{i}\right\}$ are bases for the same space, $\xi_{1}=\sum \alpha_{i} \zeta_{i}$.

Case 1. If $\alpha_{1} \neq 0$, then $F_{1}^{*}\left(z-H_{D}\right)^{-1} F_{2}$ is uniformly bounded and analytic for $z \in \mathbf{C} \backslash \sigma\left(H_{D}\right)$, with norm continuous boundary values as $z$ approaches $\sigma\left(H_{D}\right)$ from above and below.

Case 2. If $\alpha_{1}=0$, then the same conclusions hold, except that the boundary values are only strongly continuous.

Proof. For $\operatorname{Im} z>0$,

$$
\left(z-H_{D}\right)^{-1}=-i \int_{0}^{\infty} e^{-i t H_{D}} e^{i t z} d t .
$$

$e^{-i t H_{D}}=e^{-i t A} \otimes e^{-i t B}$ on $L^{2}\left(\mathbf{R}^{m}\right) \otimes L^{2}\left(\mathbf{R}^{(N-2) m}\right)$, where the first factor denotes functions of $\zeta_{1} . A$ is a negative multiple of the Laplacian, and $e^{-i t A}$ has an explicitly known integral kernel [22]. From the form of the integral kernel, an interpolation argument shows that $e^{-i t A}$ is bounded from $L^{r}\left(\mathbf{R}^{m}\right)$ to $L^{s}\left(\mathbf{R}^{m}\right)$ when $1<r<2$ and $r^{-1}+s^{-1}=1$. Moreover, the norm of $e^{-i t}$ is dominated by $(c t)^{-m\left(r^{-1}-2^{-1}\right)}$.

Case 1 . Since $e^{-i t B}$ is unitary and commutes with $F_{1}^{*}$,

$$
\left\|F_{1}^{*} e^{-i t H_{D}} F_{2}\right\|=\left\|F_{1}^{*} e^{-i t A} F_{2}\right\| \text {. }
$$

To compute $\left\|F_{1}^{*} e^{-i t A} F_{2} \phi\right\|$, fix $\zeta_{2}, \zeta_{3}, \ldots, \zeta_{k-1}$, and compute the $L^{2}$ - norm in the $\zeta_{1}$ variable first.

Hölder's inequality shows that $F_{1}$ and $F_{2}$ belong to $L^{q}\left(\mathbf{R}^{m}\right)$ for $m-\varepsilon<q<m$ $+\varepsilon$. With $\zeta_{2}, \ldots, \zeta_{k-1}$ fixed, $F_{2}\left(\sum \alpha_{i} \zeta_{i}\right) \phi\left(\zeta_{1}, \ldots, \zeta_{k-1}\right)$ belongs to $L^{r}\left(\mathbf{R}^{m}, d \zeta_{1}\right)$, where $r^{-1}=q^{-1}+\frac{1}{2} \cdot e^{-i t A}$ maps this into $L^{s}\left(\mathbf{R}^{m}, d \zeta_{1}\right)$, where $s=\frac{1}{2}-q^{-1}$. Multiplication by $\overline{F_{1}\left(\zeta_{1}\right)}$ then maps this back to $L^{2}\left(\mathbf{R}^{m}, d \zeta_{1}\right)$. The result is

$$
\begin{aligned}
& \int\left|\left(\bar{F}_{1} e^{-i t A} F_{2} \phi\right)\left(\zeta_{1}, \ldots, \zeta_{k-1}\right)\right|^{2} d \zeta_{1} \\
& \quad<(c t)^{-2 m / q}\left\|F_{1}\right\|_{q}^{2}\left\|F_{2}\right\|_{q}^{2} \alpha_{1}^{-2 m / q} \int\left|\phi\left(\zeta_{1}, \ldots, \zeta_{k-1}\right)\right|^{2} d \zeta_{1}
\end{aligned}
$$

By integrating over $\zeta_{2}, \ldots, \zeta_{k-1}$, we obtain

$$
\left\|F_{1}^{*} e^{-i t A} F_{2} \phi\right\|<\left(\alpha_{1} c t\right)^{-m / q}\left\|F_{1}\right\|_{q}\left\|F_{2}\right\|_{q}\|\phi\|_{2}
$$

Since this is valid for $q \varepsilon(m-\varepsilon, m+\varepsilon),\left\|F_{1}^{*} e^{-i t A} F_{2}\right\|$ is an $L^{1}$ function of $t$.

For $\operatorname{Im} z>0$,

$$
F_{1}^{*}\left(z-H_{D}\right)^{-1} F_{2}=-i \int_{0}^{\infty} F_{1}^{*} e^{-i t H_{D}} F_{2} e^{i t z} d t
$$

Thus,

$$
\left\|F_{1}^{*}\left(z-H_{D}\right)^{-1} F_{2}\right\|<\int_{0}^{\infty}\left\|F_{1}^{*} e^{-i t A} F_{2}\right\| d t<C .
$$


Also,

$$
\begin{array}{r}
\left\|F_{1}\left[\left(z-H_{D}\right)^{-1}-\left(w-H_{D}\right)^{-1}\right] F_{2}\right\| \\
\quad<\int_{0}^{\infty}\left\|F_{1}^{*} e^{-i t A} F_{2}\right\| e^{i t z}-e^{i t w} \mid d t
\end{array}
$$

when $\operatorname{Im} z>0$ and $\operatorname{Im} w>0$. The norm Hölder continuity now follows by the bound: $\left|e^{i t z}-e^{i t w}\right|<\operatorname{Min}(2, t|z-w|)$.

Case 2. It follows from Case 1 that $F_{1}^{*}\left(z-H_{D}\right)^{-1} F_{1}$ and $F_{2}^{*}\left(z-H_{D}\right)^{-1} F_{2}$ are uniformly bounded. Thus, $F_{1}^{*}$ and $F_{2}^{*}$ are $H_{D}$-smooth.

Since $\alpha_{1}=0$,

$$
e^{-i t H_{D}}=e^{-i t A} \otimes e^{-i t B} \otimes e^{-i t C}
$$

on $L^{2}\left(\mathbf{R}^{m}, d \zeta_{1}\right) \otimes L^{2}\left(\mathbf{R}^{m}, d \xi_{1}\right) \otimes L^{2}\left(\mathbf{R}^{(N-3) m}\right) . A$ and $B$ are negative multiples of the Laplacian; $C$ is selfadjoint.

For $\phi, \psi \in \mathcal{K}$ and $\operatorname{Im} z>0$,

$$
\begin{aligned}
\left\langle\phi, F_{1}^{*}\left(z-H_{D}\right)^{-1} F_{2} \psi\right\rangle & =-i \int_{0}^{\infty}\left\langle\phi, F_{1}^{*} e^{-i t H_{D}} F_{2} \psi\right\rangle e^{i t z} d t \\
& =-i \int_{0}^{\infty}\left\langle e^{i t B} \phi, F_{1}^{*} e^{-i t A} e^{-i t C} F_{2} \psi\right\rangle e^{i t z} d t \\
& =-i \int_{0}^{\infty}\left\langle F_{2}^{*} e^{i t B} \phi, F_{1}^{*} e^{-i t A} e^{-i t C} \psi\right\rangle e^{i t z} d t
\end{aligned}
$$

Hence,

$$
\begin{aligned}
\left|\left\langle\phi, F_{1}^{*}\left(z-H_{D}\right)^{-1} F_{2} \psi\right\rangle\right| & <\left(\int_{0}^{\infty}\left\|F_{2}^{*} e^{i t B} \phi\right\|^{2} d t\right)^{1 / 2}\left(\int_{0}^{\infty}\left\|F_{1}^{*} e^{-i t A} \psi\right\|^{2} d t\right)^{1 / 2} \\
& =\left(\int_{0}^{\infty}\left\|F_{2}^{*} e^{i t H_{D}} \phi\right\|^{2} d t\right)^{1 / 2}\left(\int_{0}^{\infty}\left\|F_{1}^{*} e^{-i t H_{D}} \psi\right\|^{2} d t\right)^{1 / 2} \\
& <\left\|F_{2}^{*}\right\|_{H_{D}}\left\|F_{1}^{*}\right\|_{H_{D}}\|\phi\|\|\psi\| .
\end{aligned}
$$

Here we have used the Schwarz and Hölder inequalities and Lemma II.1.

To prove the strong continuity, the same type of computation shows:

$$
\begin{aligned}
\mid\left\langle\phi, F_{1}^{*}\left[\left(z-H_{D}\right)^{-1}\right.\right. & \left.\left.-\left(w-H_{D}\right)^{-1}\right] F_{2} \psi\right\rangle \mid \\
& <\left(\int_{0}^{\infty}\left\|F_{2}^{*} e^{i t H_{D}} \phi\right\|^{2} d t\right)^{1 / 2}\left(\int_{0}^{\infty}\left\|F_{1}^{*} e^{-i t H_{D}} \psi\right\|^{2}\left|e^{i t z}-e^{i t w}\right|^{2} d t\right)^{1 / 2} \\
& <\left\|F_{2}^{*}\right\|_{H_{D}}\|\phi\|\left(\int_{0}^{\infty}\left\|F_{1}^{*} e^{-i t H_{D}} \psi\right\|^{2}\left|e^{i t z}-e^{i t w}\right|^{2} d t\right)^{1 / 2} .
\end{aligned}
$$

Hence,

$$
\begin{aligned}
\left\|F_{1}^{*}\left[\left(z-H_{D}\right)^{-1}-\left(w-H_{D}\right)^{-1}\right] F_{2} \psi\right\| \\
\quad<\left\|F_{2}^{*}\right\|_{H_{D}}\left(\int_{0}^{\infty}\left\|F_{1}^{*} e^{-i t H_{D}} \psi\right\|^{2}\left|e^{i t z}-e^{i t w}\right|^{2} d t\right)^{1 / 2} .
\end{aligned}
$$

By the dominated convergence theorem, the integral tends to zero as $z$ approaches $w$.

The proof for $\operatorname{Im} z<0$ is similar. 
LEMMA II.4. Suppose $F_{1}$ and $F_{2}$ satisfy the hypotheses of Lemma II.3. Then

$$
\begin{aligned}
F_{1}^{*}[(\lambda & \left.\left.+i 0-H_{D}\right)^{-1}-\left(\lambda-i 0-H_{D}\right)^{-1}\right] F_{2} \\
& =F_{1}^{*}\left[\left(\lambda+i 0-H_{D}\right)^{-1}-\left(\lambda-i 0-H_{D}\right)^{-1}\right] E^{D}(a, b) F_{2}
\end{aligned}
$$

for any $\lambda \in(a, b) . E^{D}(a, b)$ denotes the spectral projection for $H_{D}$ corresponding to the interval $(a, b)$.

Proof. If $F_{1}$ and $F_{2}$ are bounded, the result is trivial because

$$
\left[\left(\lambda+i 0-H_{D}\right)^{-1}-\left(\lambda-i 0-H_{D}\right)^{-1}\right]\left(1-E^{D}(a, b)\right)=0 \text {. }
$$

More general functions $F_{1}$ and $F_{2}$ are limits of bounded functions. By taking such limits, the lemma follows from the bounds of Lemma II.3.

D. Fourier restriction theorems (Agmon [1], Kuroda [17]).

Definition. For $k>0$, define $\pi(k): \delta\left(\mathbf{R}^{m}\right) \rightarrow L^{2}\left(S^{m-1}, d \omega\right)$ by $(\pi(k) f)(\omega)=$ $k^{1 / 2(m-1)} \hat{f}(k \omega)$, where $\omega$ belongs to the unit sphere $S^{m-1}, \hat{f}$ is the Fourier transform of $f$, and $d \omega$ is the invariant surface measure on $S^{m-1} \subseteq \mathbf{R}^{m}$.

LemMa II.5. Suppose $1 \leqslant p \leqslant 2, m \geqslant 3$, and $1 / 2<\delta<m / 2$. If $\delta(p)=2 \delta(1-$ $\left.p^{-1}\right)$, then $\pi(k)$ extends to a bounded mapping of $L_{\delta(p)}^{P}\left(\mathbf{R}^{m}\right)$ into $L^{2}\left(S^{m-1}, d \omega\right)$. The norm of this mapping is bounded by

$$
\operatorname{Min}\left\{c_{1} k^{(m-1)\left(p^{-1}-1 / 2\right)}, c_{2} k^{\left[(m-1)\left(p^{-1}-1 / 2\right)+(\delta-1 / 2)\left(2-2 p^{-1}\right)\right]}\right\},
$$

where $c_{1}$ and $c_{2}$ depend only on $p$. Moreover, if $p>1$, then $k \mapsto \pi(k)$ is norm Hölder continuous.

Proof. When $p=2$, these results may be found in [7] or [8].

When $p=1$, norm continuity in $k$ does not hold, but the other results are trivial because $\hat{f}$ is continuous and $\|\hat{f}\|_{\infty} \leqslant c\|f\|_{1}$.

The results now follow by interpolation (see Stein [35]) between $L^{1}$ and $L_{\delta}^{2}$. Note that Hölder continuity of order $\theta$ on $L_{\delta}^{2}$ and boundedness on $L^{1}$ imply Hölder continuity of order $2 \theta\left(1-p^{-1}\right)$ on $L_{\delta(p)}^{P}$ for $1<p<2$.

Proposition II.6. Suppose $m_{1}>3, \delta>1,1<p<2$, and $\delta(p)=2 \delta\left(1-p^{-1}\right)$. $\pi(k)$ extends to a bounded mapping of $L_{\delta(p)}^{P}\left(\mathbf{R}^{m_{1}}\right) \otimes L^{2}\left(\mathbf{R}^{m_{2}}\right)$ into $L^{2}\left(S^{m_{1}+m_{2}-1}, d \omega\right)$. The mapping $k \mapsto \pi(k)$ is strongly continuous and uniformly bounded in norm when $k$ is restricted to a bounded subset of $(0, \infty)$.

Proof (Ginibre and Moulin [7]). Choose $f \in \mathcal{S}\left(\mathbf{R}^{m_{1}+m_{2}}\right)$, and let $p_{1}$ and $p_{2}$ denote the Fourier conjugate variables to $x_{1} \in \mathbf{R}^{m_{1}}$ and $x_{2} \in \mathbf{R}^{m_{2}}$. Decompose $p_{1}$ into radial and angular variables $\left|p_{1}\right|$ and $\omega_{1}$.

If $k_{1}=\left(k^{2}-\left|p_{2}\right|^{2}\right)^{1 / 2}$ and $m=m_{1}+m_{2}$, then

$$
\begin{aligned}
\|\pi(k) f\|_{L^{2}\left(S^{m-1}\right)}^{2} & =k^{(m-1)} \int_{S^{m-1}}|\hat{f}(k \omega)|^{2} d \omega \\
& =\int_{\mathbf{R}^{m}} \delta(k-|p|)|\hat{f}(p)|^{2} d p .
\end{aligned}
$$


Change variables to $\left|p_{1}\right|, \omega_{1}$, and $p_{2}$, and compute the $\left|p_{1}\right|$ integral:

$$
\begin{aligned}
\|\pi(k) f\|_{L^{2}\left(S^{m-1}\right)}^{2} & =k \int_{\left|p_{2}\right|<k} d p_{2} k_{1}^{\left(m_{1}-2\right)} \int_{S^{m_{1}-1}}\left|\hat{f}\left(k_{1} \omega_{1}, p_{2}\right)\right|^{2} d \omega_{1} \\
& =k \int_{\left|p_{2}\right|<k} d p_{2} k_{1}^{-1}\left\|\pi_{1}\left(k_{1}\right) \tilde{f}\left(\cdot, p_{2}\right)\right\|_{L^{2}\left(S^{m_{1}-1}\right)}^{2}
\end{aligned}
$$

where the last norm is computed for fixed $p_{2}, \tilde{f}$ denotes partial Fourier transform in the second variable, and $\pi_{1}\left(k_{1}\right)$ denotes the Fourier restriction map in the first variable.

By Lemma II.5, $k_{1}^{-1}\left\|\pi_{1}\left(k_{1}\right)\right\|^{2}$ is uniformly bounded for $k_{1} \in(0, k)$ from $L_{\delta(p)}^{P}\left(\mathbf{R}^{m_{1}}\right)$ into $L^{2}\left(S^{m_{1}-1}, d \omega_{1}\right)$, so

$$
\begin{aligned}
\|\pi(k) f\|_{L^{2}\left(S^{m-1}\right)}^{2} & \leqslant k \int_{\left|p_{2}\right|<k} d p_{2} k_{1}^{-1}\left\|\pi_{1}\left(k_{1}\right)\right\|^{2}\left\|f\left(\cdot, p_{2}\right)\right\|_{L \delta_{(p)}\left(\mathbf{R}^{\left.m_{1}\right)}\right)}^{2} \\
& \leqslant k C(k) \int_{\left|p_{2}\right|<k} d p_{2}\left\|\tilde{f}\left(\cdot, p_{2}\right)\right\|_{L_{\delta(p)}}^{2} \\
& =k C(k) \int d x_{2}\left\|f\left(\cdot, x_{2}\right)\right\|_{L_{\delta(p)}}^{2} \\
& =k C(k)\|f\|_{L \delta_{(p)} \otimes L^{2}}^{2} .
\end{aligned}
$$

This implies the uniform boundedness for $k$ restricted to a bounded subset of $(0, \infty)$. The strong continuity of $\pi(k)$ follows from the norm continuity of $\pi_{1}\left(k_{1}\right)$.

E. Dilation analyticity (Balslev and Combes [3], see also van Winter [38], [39]).

The unitary group of dilations on $L^{2}\left(\mathbf{R}^{n}\right)$ is given by $(U(\theta) f)(x)=e^{n \theta / 2} \psi\left(e^{\theta} x\right)$. A simple computation shows $H_{0}(\theta)=U(\theta) H_{0} U(-\theta)=e^{-2 \theta} H_{0}$ for all $\theta \in \mathbf{R}$. $H_{0}(\theta)$ clearly extends to an analytic family $H_{0}(\theta)$ for all $\theta \in \mathbf{C}$.

Following Simon's treatment [30] of dilation analyticity for quadratic forms, we make the following definitions.

Definition. Suppose $\alpha>0$. A compact operator $v$ : $\mathcal{H}_{+1} \rightarrow \mathcal{K}_{-1}$ belongs to the class $\mathcal{F}_{\alpha}$, if and only if the family of operators $v(\theta)=U(\theta) v U(-\theta)$ has an analytic continuation to $\{\theta:|\operatorname{Im} \theta|<\alpha\}$ as operators from $\mathcal{K}_{+1}$ into $\mathcal{K}_{-1}$. If $v(\theta)$ : $\mathscr{H}_{+1} \rightarrow \mathcal{H}_{-1}$ is norm continuous in $\{\theta:|\operatorname{Im} \theta|<\alpha\}$ and analytic in $\{\theta:|\operatorname{Im} \theta|<$ $\alpha\}$, then $v$ belongs to the class $\overline{\mathscr{F}}_{\alpha}$.

If $v \in \mathscr{F}_{\alpha}$ for some $\alpha>0$, then $v$ is called dilation analytic.

If $H=H_{0}+\Sigma_{i<j} V_{i j}$, where $V_{i j}=v_{i j} \otimes 1$ and each $v_{i j} \in \mathscr{F}_{\alpha}$, then $H(\theta)=H_{0}(\theta)$ $+\Sigma_{i<j} V_{i j}(\theta)$ may be defined by quadratic form methods. If $D$ is a cluster decomposition, then $H_{D}(\theta)$ and the cluster Hamiltonians, $h_{i}(\theta)$ may be defined.

Let $D$ be a cluster decomposition, with cluster Hamiltonians $h_{i}(1<i<k)$. If the potentials are dilation analytic, then we define $\Sigma_{D}(\theta)=\left\{E_{1}+E_{2}\right.$ $+\cdots+E_{k}: E_{i}$ is an eigenvalue of $\left.h_{i}(\theta)\right\}$ and $\Sigma(\theta)=\{0\} \cup\left(\cup \#(D)>2 \Sigma_{D}(\theta)\right)$ is the set of thresholds of $H(\theta)$. 
Theorem II.7 (BALslev AND Combes [3], Simon [30]). Suppose the two body potentials $V_{i j}=v_{i j} \otimes 1$ have $v_{i j} \in \mathscr{F}_{\alpha}$ and are selfadjoint. The essential spectrum of $H(\theta)$ is $\sigma_{\text {ess }}(H(\theta))=\left\{z+e^{-2 \theta} r: z \in \Sigma(\theta), r \in[0, \infty)\right\}$. If $\mu=$ inf $\Sigma(0)$, then the discrete spectrum of $H(\theta)$ is contained in $\mathbf{R} \cup\{\mu+z:-2 \operatorname{Im} \theta<\arg z<0\}$ if $\operatorname{Im} \theta \geqslant 0$, and $\mathbf{R} \cup\{\mu+z: 0<\arg z<-2 \operatorname{Im} \theta\}$ if $\operatorname{Im} \theta<0$. The discrete spectrum of $H(\theta)$ can accumulate only at points of $\Sigma(\theta)$. The nonthreshold eigenvalues of $H$ are the real nonthreshold eigenvalues of $H(\theta)$, and $\Sigma(\theta) \cap \mathbf{R}=\Sigma(0)$ whenever $|\operatorname{Im} \theta|<\pi / 2$.

TheOREM II.8 (BALSLEV AND Combes [3]). Suppose $H=H_{0}+\Sigma_{i<j} V_{i j}$, where the two body potentials are dilation analytic. Then $H$ has empty singular continuous spectrum.

The following theorem proves exponential fall-off of bound states corresponding to nonthreshold eigenvalues. Without dilation analyticity, O'Connor [21] has proven this fact for bound states corresponding to discrete eigenvalues.

Theorem II.9 (Combes AND Thomas [4]). Suppose $H=H_{0}+\Sigma_{i<j} V_{i j}$ is selfadjoint, with dilation analytic potentials. If $H \psi=E \psi$ and $E \notin \Sigma(0)$, then $\psi(x)$ lies in the domain of multiplication by $e^{a|x|}$ for some $a>0$. Moreover, $e^{a|x|} \psi(x)$ belongs to the operator domain of $\mathrm{H}_{0}^{1 / 2}$ for some $a>0$.

Proof. Combes and Thomas [4] prove all but the last statement. Simon [34] proves $e^{a|x|} \psi(x) \in D\left(H_{0}^{1 / 2}\right)$.

Theorem II.10 (Balslev [2], Simon [31], [32]). Suppose $H=H_{0}+\Sigma_{i<j} V_{i j}$ is selfadjoint. If $N=2$ and $V \in \mathscr{F}_{\alpha}$ for some $\alpha>0$, or if $N \geqslant 3$ and each $V_{i j}=v_{i j} \otimes$ 1 , with $v_{i j} \in \overline{\mathscr{F}}_{\pi / 2}$, then $H$ has no positive eigenvalues.

LEMMA II.11. Suppose $M(z, \theta)$ is an analytic operator valued function in the region $\left\{(z, \theta) \in \mathbf{C}^{2}:|\operatorname{Im} \theta|<\alpha, z \in \mathbf{C} \backslash \sigma_{\text {ess }}(H(\theta))\right\}$, with norm continuous boundary values as $z$ approaches $\sigma_{\text {ess }}(H(\theta))$. Suppose $U(\phi) M(z, \theta) U(-\phi)=M(z, \theta+\phi)$ for $\phi \in \mathbf{R}$, and assume $(M(z, \theta))^{2}$ is compact. If $E \in \mathbf{R}$, then $1 \in \sigma(M(E+i 0,0))$, if and only if $1 \in \sigma(M(E, \theta))$ for all $\theta$ with $0<\operatorname{Im} \theta<\operatorname{Min}(\alpha, \pi / 2)$.

Proof. Since $(M(z, \theta))^{2}$ is compact, $1 \in \sigma(M(z, \theta))$ if and only if $1 \in$ $\sigma\left((M(z, \theta))^{n}\right)$ for all $n \geqslant 2$. Thus, it suffices to prove $1 \in \sigma\left((M(E+i 0,0))^{n}\right)$ if and only if $1 \in \sigma\left((M(E, \theta))^{n}\right)$ for all $n \geqslant 2$.

If $1 \in \sigma\left((M(E, \theta))^{n}\right)$ whenever $0<\operatorname{Im} \theta<\operatorname{Min}(\alpha, \pi / 2)$, then $1 \in \sigma((M(E+$ $\left.i 0,0))^{n}\right)$ by continuity.

Next, suppose $1 \in \sigma\left((M(E+i 0,0))^{n}\right)$ but $1 \notin \sigma\left((M(E, \phi))^{n}\right)$ for some $n>2$ and $\phi$ with $\operatorname{Im} \phi$ small and positive. Since $M(E+i 0, \theta)=U(\theta) M(E+$ $i 0,0) U(-\theta)$ for $\theta \in \mathbf{R}, 1 \in \sigma\left((M(E+i 0, \theta))^{n}\right)$ for all $\theta \in \mathbf{R}$.

Since $1 \notin \sigma\left((M(E, \phi))^{n}\right)$, the set of $\theta \in \mathbf{R}$ such that $1 \in \sigma\left((M(E+i 0, \theta))^{n}\right)$ must be a closed set of measure zero by a theorem of Kuroda [16] (see also Simon [29, p. 127]).

This contradiction proves the lemma. 


\section{F. Existence of wave operators.}

The existence of channel wave operators has been proved by Schechter [25] and Simon [33] for the types of potentials which concern us. Simon's proof is an extension of a simple method due to Cook [5].

TheOREM II.12 (Simon [33]). Suppose the two body potentials $V_{i j}$ belong to $L P\left(\mathbf{R}^{m}\right)+L_{\delta}^{\infty}\left(\mathbf{R}^{m}\right)$ for some $p>m / 2$ and $\delta>2$. Then the channel wave operators

$$
\Omega_{\alpha}^{\mp}=\text { strong- } \lim _{t \rightarrow \pm \infty} e^{i t H} e^{-i t T_{\alpha} P_{\alpha}}
$$

exist.

III. Many-body Kuroda completeness as a stationary problem. This section is devoted to proving Theorem III.1, which reduces the many-body Kuroda completeness problem to the question of finding an appropriate formula for the resolvent $(z-H)^{-1}$. The idea of this theorem is not new. Implicitly, it appears in the works of Faddeev [6], Hepp [10], Ginibre and Moulin [7], Thomas [36], and Sigal [26]; explicitly, it may be found in Howland [11].

Definition. Suppose $H$ is a many-body Hamiltonian. The multiparticle limiting absorption principle holds for $H$, if

$$
(z-H)^{-1}=\sum_{D}\left(z-H_{D}\right)^{-1} P_{D} \sum_{l=1}^{L(D)} F_{l, D} Z_{l, D}(z),
$$

where

(a) there exists $\delta_{0}$ such that $\phi \in L_{\delta_{0}}^{2}\left(\mathbf{R}^{(N-1) m}\right)$ implies $Z_{l, D}(z) \phi$ is an $L^{2}\left(\mathbf{R}^{(N-1) m}\right)$ valued meromorphic function in $\mathrm{C} \backslash \sigma_{\text {ess }}(H)$, with continuous extensions to $\sigma_{\text {ess }}(H)$ from above and below, in the complement of a closed set $\mathcal{E}$ of measure zero; and

(b) for each $l$ and $D, F_{l, D}$ maps $L^{2}\left(\mathbf{R}^{(N-1) m}\right)$ into $L_{\mu}^{p}\left(\mathbf{R}^{m}\right) \otimes L^{2}\left(\mathbf{R}^{(N-2) m}\right)$, for some $\mu>1$ and $p \in(1,2$ ], where the first factor denotes functions of a Jacobi coordinate for the motion of the centers of mass of the clusters of $D$.

THEOREM III.1. Suppose the multiparticle limiting absorption principle holds for $\boldsymbol{H}$ on $L^{2}\left(\mathbf{R}^{(N-1) m}\right)$, where $m \geqslant 3$. If the channel wave operators exist, then they are complete in the sense that $P_{\text {a.c. }}=\Sigma_{\alpha} \Omega_{\alpha}^{ \pm} \Omega_{\alpha}^{ \pm *}$.

As a first step toward the proof of this theorem, we prove several lemmas related to the orthogonality of channels. Next, using the limiting absorption principle, we obtain a formula for $\left(\Omega_{\alpha}^{ \pm *} \phi\right)^{\hat{n}}$, when $\phi \in L_{\delta_{0}}^{2}\left(\mathbf{R}^{(N-1) m}\right)$. This and Stone's formula for spectral projections are then used to prove Theorem III.1.

Definition. Let $H_{1}$ and $H_{2}$ be selfadjoint operators on $\mathcal{H}$. For each $\varepsilon>0$ and each Borel set $A \subseteq \mathbf{R}$, the operators $\Omega^{ \pm}\left(\varepsilon, H_{2}, H_{1}, A\right)$ are defined by

$$
\left\langle\phi, \Omega^{\mp}\left(\varepsilon, H_{2}, H_{1}, A\right) \psi\right\rangle=\frac{\varepsilon}{\pi} \int_{A}\left\langle\left(\lambda \pm i \varepsilon-H_{2}\right)^{-1} \phi,\left(\lambda \pm i \varepsilon-H_{1}\right)^{-1} \psi\right\rangle d \lambda .
$$

LEMma III.2 (Howland [11]). Let $H_{1}$ and $H_{2}$ be selfadjoint on $\mathcal{H}$. $\Omega^{\mp}\left(\varepsilon, H_{2}, H_{1}, A\right)$ are well-defined contractions on $\mathcal{H}$ for all Borel sets $A \subseteq \mathbf{R}$ and all $\varepsilon>0$. If $H_{1}$ has purely absolutely continuous spectrum and spectral projections $E_{1}(\cdot)$, then $E_{1}(A) \psi=0$ implies $\lim _{e \downarrow 0} \Omega^{\mp}\left(\varepsilon, H_{2}, H_{1}, A\right) \psi=0$. 
Proof. Let $\delta_{e}(t)=(\varepsilon / \pi) /\left(t^{2}+\varepsilon^{2}\right)$.

The Schwarz inequality shows that the absolute value of the integrand appearing in the definition of $\Omega^{\mp}\left(\varepsilon, H_{2}, H_{1}, A\right)$ is bounded by

$$
\begin{aligned}
(\varepsilon / \pi)\left\|\left(\lambda \pm i \varepsilon-H_{2}\right)^{-1} \phi\right\|\left\|\left(\lambda \pm i \varepsilon-H_{1}\right)^{-1} \psi\right\| \\
=\left((\varepsilon / \pi)\left\|\left(\lambda \pm i \varepsilon-H_{2}\right)^{-1} \phi\right\|^{2}\right)^{1 / 2}\left((\varepsilon / \pi)\left\|\left(\lambda \pm i \varepsilon-H_{1}\right)^{-1} \psi\right\|^{2}\right)^{1 / 2} \\
=\left(\left\langle\phi, \delta_{e}\left(\lambda-H_{2}\right) \phi\right\rangle\right)^{1 / 2}\left(\left\langle\psi, \delta_{e}\left(\lambda-H_{1}\right) \psi\right\rangle\right)^{1 / 2} .
\end{aligned}
$$

So, by the Schwarz inequality and Fubini's theorem,

$$
\begin{aligned}
\left\langle\phi, \Omega^{\mp}\right. & \left.\left(\varepsilon, H_{2}, H_{1}, A\right) \psi\right\rangle\left.\right|^{2} \\
& <\left(\int_{A}\left\langle\phi, \delta_{\varepsilon}\left(\lambda-H_{2}\right) \phi\right\rangle d \lambda\right)\left(\int_{A}\left\langle\psi, \delta_{\varepsilon}\left(\lambda-H_{1}\right) \psi\right\rangle d \lambda\right) \\
& =\left(\int_{\mathbf{R}}\left(\delta_{\varepsilon} * \chi_{A}\right)(\lambda) d\left\langle\phi, E_{2}(\lambda) \phi\right\rangle\right)\left(\int_{\mathbf{R}}\left(\delta_{\varepsilon} * \chi_{A}\right)(\lambda) d\left\langle\psi, E_{1}(\lambda) \psi\right\rangle\right) .
\end{aligned}
$$

Since $\int_{\mathbf{R}} \delta_{e}(\lambda) d t=1$ and $\left\|\chi_{A}\right\|_{\infty} \leqslant 1$, we have $0 \leqslant \delta_{e} * \chi_{A} \leqslant 1$. So, the right-hand side of (III.1) is bounded by $\|\phi\|^{2}\|\psi\|^{2}$. The operators $\Omega^{\mp}\left(\varepsilon, H_{2}, H_{1}, A\right)$ are, therefore, well-defined contractions.

The measure $d\left\langle\psi, E_{1}(\cdot) \psi\right\rangle$ is absolutely continuous with respect to Lebesgue measure. As a consequence ([37, pp. 28-31]), $\lim _{e \downarrow 0} \delta_{\varepsilon} * \chi_{A}=\chi_{A}$ a.e. with respect to $d\left\langle\psi, E_{1}(\cdot) \psi\right\rangle$. Moreover, $0 \leqslant \delta_{e} * \chi_{A} \leqslant 1$, so the dominated convergence theorem shows:

$$
\lim _{\varepsilon \downarrow 0} \int_{\mathbf{R}}\left(\delta_{e} * \chi_{A}\right)(\lambda) d\left\langle\psi, E_{1}(\lambda) \psi\right\rangle=\int_{\mathbf{R}} \chi_{A}(\lambda) d\left\langle\psi, E_{1}(\lambda) \psi\right\rangle=\left\|E_{1}(A) \psi\right\|^{2} .
$$

Since

$$
0 \leqslant \int_{\mathbf{R}}\left(\delta_{\varepsilon} * \chi_{A}\right)(\lambda) d\left\langle\phi, E_{2}(\lambda) \phi\right\rangle<\|\phi\|^{2}
$$

(III.1) shows

$$
\underset{\varepsilon \downarrow 0}{\lim \sup }\left\|\Omega^{\mp}\left(\varepsilon, H_{2}, H_{1}, A\right) \psi\right\|^{2} \leqslant\left\|E_{1}(A) \psi\right\|^{2} .
$$

Therefore, $E_{1}(A) \psi=0$ implies $\lim _{\varepsilon \downarrow 0} \Omega^{\mp}\left(\varepsilon, H_{2}, H_{1}, A\right) \psi=0$.

LEMMA III.3. Let $H_{1}$ and $H_{2}$ be selfadjoint on $\mathcal{H}$. For all $\varepsilon>0$,

$$
\Omega^{\mp}\left(\varepsilon, H_{2}, H_{1}, \mathbf{R}\right) \psi=2 \varepsilon \int_{0}^{ \pm \infty} e^{\mp 2 e t} e^{i t H_{2}} e^{-i t H_{H}} \psi d t .
$$

Proof. Consider only $\Omega^{-}$; the proof for $\Omega^{+}$is similar. Fix $\phi, \psi \in \mathcal{K}$ and define $\phi(\lambda)=(\varepsilon / \pi)^{1 / 2}\left(\lambda+i \varepsilon-H_{2}\right)^{-1} \phi$ and $\psi(\lambda)=(\varepsilon / \pi)^{1 / 2}\left(\lambda+i \varepsilon-H_{1}\right)^{-1} \psi$. Then, by explicit computation,

$$
\hat{\phi}(t)=(2 \varepsilon)^{1 / 2} i \chi_{(0, \infty)}(t) e^{-e t} e^{-i t H_{2} \phi}
$$

and

$$
\hat{\psi}(t)=(2 \varepsilon)^{1 / 2} i \chi_{(0, \infty)}(t) e^{-\varepsilon t} e^{i t H} \psi
$$


These formulas and Lemma II.1 show

$$
\begin{aligned}
\left\langle\phi, \Omega^{-}\left(\varepsilon, H_{2}, H_{1}, \mathbf{R}\right) \psi\right\rangle & =\int_{\mathbf{R}}\langle\phi(\lambda), \psi(\lambda)\rangle d \lambda \\
& =\int_{\mathbf{R}}\langle\hat{\phi}(t), \hat{\psi}(t)\rangle d t \\
& =2 \varepsilon \int_{0}^{\infty}\left\langle\phi, e^{-2 e t} e^{i t H_{2}} e^{-i t H} \psi\right\rangle d t \\
& =\left\langle\phi, 2 \varepsilon \int_{0}^{\infty} e^{-2 e t} e^{i t H_{2}} e^{-i t H_{H}} \psi d t\right\rangle .
\end{aligned}
$$

LEMMA III.4 (HowLAND [11]). Let $H_{1}$ and $H_{2}$ be commuting selfadjoint operators on $\mathcal{H C}$. Assume $\mathrm{H}_{1}$ has purely absolutely continuous spectrum and that $\mathrm{H}=\mathrm{H}_{1}-\mathrm{H}_{2}$ has kernel $\{0\}$. Then, for Borel sets $A \subseteq \mathbf{R}$,

$$
\underset{\varepsilon \downarrow 0}{\text { weak-lim }} \Omega^{\mp}\left(\varepsilon, H_{2}, H_{1}, A\right)=0 .
$$

Proof.

$$
\begin{aligned}
\Omega^{\mp}\left(\varepsilon, H_{2}, H_{1}, A\right)= & \Omega^{\mp}\left(\varepsilon, H_{2}, H_{1}, \mathbf{R}\right) E_{1}(A)-\Omega^{\mp}\left(\varepsilon, H_{2}, H_{1}, \mathbf{R} \backslash A\right) E_{1}(A) \\
& +\Omega^{\mp}\left(\varepsilon, H_{2}, H_{1}, A\right) E_{1}(\mathbf{R} \backslash A),
\end{aligned}
$$

where $H_{1}$ has spectral projections $E_{1}(\cdot)$.

The last two terms in this expression converge strongly to zero as $\varepsilon \rightarrow 0$ by Lemma III.2. Thus, it suffices to show weak- $\lim _{e \downarrow 0} \Omega^{\mp}\left(\varepsilon, H_{2}, H_{1}, \mathbf{R}\right)=0$.

By Lemma III.3,

$$
\begin{aligned}
\left\langle\phi, \Omega^{\mp}\left(\varepsilon, H_{2}, H_{1}, \mathbf{R}\right) \psi\right\rangle & =2 \varepsilon \int_{0}^{ \pm \infty} e^{\mp 2 e t}\left\langle\phi, e^{i t H_{2}} e^{-i t H_{4}} \psi\right\rangle d t \\
& =2 \varepsilon \int_{0}^{ \pm \infty} e^{\mp 2 e t}\left\langle\phi, e^{-i t H} \psi\right\rangle d t \\
& = \pm 2 i \varepsilon\left\langle\phi,(H \mp 2 i \varepsilon)^{-1} \psi\right\rangle .
\end{aligned}
$$

As $\varepsilon$ tends to zero, this quantity converges to $-\left\langle\phi, P_{0} \psi\right\rangle$, where $P_{0}$ is the orthogonal projection onto the kernel of $H$. Hence, the hypotheses imply it converges to zero.

LEMMA III.5. Let $\mathrm{H}_{1}$ and $\mathrm{H}_{2}$ be commuting selfadjoint operators on $\mathcal{H}$, such that $H_{1}-H_{2}$ has kernel $\{0\}$ and $H_{1}$ has purely absolutely continuous spectrum. Let $F_{1}$ and $F_{2}$ be operators on $\mathcal{C}$ such that $F_{1}^{*}$ and $F_{2}^{*}$ are Kato smooth with respect to $H_{1}$ and $H_{2}$, respectively. Suppose $\phi_{ \pm}: D_{ \pm} \rightarrow \mathcal{H}$ and $\psi_{ \pm}: D_{ \pm} \rightarrow \mathcal{H}$ are continuous functions, where $D_{ \pm}=\{z=x \pm i y \in \mathbf{C}: a<x<b, 0<y<c\}$. Then

$$
\begin{aligned}
\lim _{\varepsilon \downarrow 0} \varepsilon \int_{a}^{b}\left\langle\left(\lambda \pm i \varepsilon-H_{2}\right)^{-1}\right. & F_{2} \phi_{ \pm}(\lambda \pm i \varepsilon), \\
& \left.\left(\lambda \pm i \varepsilon-H_{1}\right)^{-1} F_{1} \psi_{ \pm}(\lambda \pm i \varepsilon)\right\rangle d \lambda=0 .
\end{aligned}
$$


Proof. Assume, without loss of generality, that $F_{1} \neq 0, F_{2} \neq 0, \phi_{ \pm} \neq 0, \psi_{ \pm} \neq 0$, $a<b, c\rangle 0$. For convenience, consider only the + signs, with $\phi=\phi_{+}, \psi=\psi_{+}$, and $D=D_{+}$.

Let $\delta>0$ be given, and choose $M$ such that $\|\phi(z)\|<M$ and $\|\psi(z)\|<M$ for all $z \in D$. Since $F_{1}$ and $F_{2}$ have dense domains, there exist simple functions $f$ : $[a, b] \rightarrow \mathscr{D}\left(F_{1}\right)$ and $g:[a, b] \rightarrow \mathscr{D}\left(F_{2}\right)$, such that $\|f(x)\|<M,\|g(x)\|<M, \| \phi(x)$ $-g(x) \|<\delta$, and $\|\psi(x)-f(x)\|<\delta$, for all $x \in[a, b]$.

By the continuity of $\phi$ and $\psi$, and the compactness of $D$, there exists $\gamma>0$, such that $0<\varepsilon<\gamma$ implies $\|\phi(\lambda+i \varepsilon)-\phi(\lambda)\|<\delta$ and $\|\psi(\lambda+i \varepsilon)-\psi(\lambda)\|<\delta$ for all $\lambda \in[a, b]$.

Thus, $\|\phi(\lambda+i \varepsilon)-g(\lambda)\|<2 \delta$ and $\|\psi(\lambda+i \varepsilon)-f(\lambda)\|<2 \delta$ for all $\lambda \in[a, b]$, whenever $0<\varepsilon<\gamma$.

Define:

$$
\begin{array}{r}
A(\varepsilon)=\varepsilon \int_{a}^{b}\left\langle\left(\lambda+i \varepsilon-H_{2}\right)^{-1} F_{2} g(\lambda),\left(\lambda+i \varepsilon-H_{1}\right)^{-1} F_{1} f(\lambda)\right\rangle d \lambda \\
B(\varepsilon)=\varepsilon \int_{a}^{b}\left\langle\left(\lambda+i \varepsilon-H_{2}\right)^{-1} F_{2} g(\lambda),\right. \\
\left.\left(\lambda+i \varepsilon-H_{1}\right)^{-1} F_{1}[\psi(\lambda+i \varepsilon)-f(\lambda)]\right\rangle d \lambda \\
C(\varepsilon)=\varepsilon \int_{a}^{b}\left\langle\left(\lambda+i \varepsilon-H_{2}\right)^{-1} F_{2}[\phi(\lambda+i \varepsilon)-g(\lambda)],\right. \\
\left.\left(\lambda+i \varepsilon-H_{1}\right)^{-1} F_{1} \psi(\lambda+i \varepsilon)\right\rangle d \lambda .
\end{array}
$$

By Lemma III.4, there exists $\gamma_{1}<\gamma$, so that $\varepsilon<\gamma_{1}$ implies $|A(\varepsilon)|<\delta$.

By Hölder's inequality and Lemma II.2, $\varepsilon<\gamma$ implies

$$
\begin{aligned}
|B(\varepsilon)|< & \varepsilon \int_{a}^{b}\left\|\left(\lambda+i \varepsilon-H_{2}\right)^{-1} F_{2} g(\lambda)\right\| \\
& \times\left\|\left(\lambda+i \varepsilon-H_{1}\right)^{-1} F_{1}[\psi(\lambda+i \varepsilon)-f(\lambda)]\right\| d \lambda \\
< & \left(\varepsilon \int_{a}^{b}\left\|\left(\lambda+i \varepsilon-H_{2}\right)^{-1} F_{2} g(\lambda)\right\|^{2} d \lambda\right)^{1 / 2} \\
& \times\left(\varepsilon \int_{a}^{b}\left\|\left(\lambda+i \varepsilon-H_{1}\right)^{-1} F_{1}[\psi(\lambda+i \varepsilon)-f(\lambda)]\right\|^{2} d \lambda\right)^{1 / 2} \\
< & \left(\frac{b-a}{\pi}\left\|F_{2}\right\|_{H_{2}}^{2}\left(\sup _{\lambda}\|g(\lambda)\|\right)^{2}\right)^{1 / 2} \\
& \times\left(\frac{b-a}{\pi}\left\|F_{1}\right\|_{H_{1}}^{2}\left(\sup _{\lambda}\|\psi(\lambda+i \varepsilon)-f(\lambda)\|\right)^{2}\right)^{1 / 2} \\
< & 2 \frac{b-a}{\pi} M\left\|F_{1}\right\|_{H_{1}}\left\|F_{2}\right\|_{H_{2}} \delta .
\end{aligned}
$$


Similarly, when $\varepsilon<\gamma$,

$$
\begin{aligned}
|C(\varepsilon)|< & \left(\varepsilon \int_{a}^{b}\left\|\left(\lambda+i \varepsilon-H_{2}\right)^{-1} F_{2}[\phi(\lambda+i \varepsilon)-g(\lambda)]\right\|^{2} d \lambda\right)^{1 / 2} \\
& \times\left(\varepsilon \int_{a}^{b}\left\|\left(\lambda+i \varepsilon-H_{1}\right)^{-1} F_{1} \psi(\lambda+i \varepsilon)\right\|^{2} d \lambda\right)^{1 / 2} \\
< & \left(\frac{b-a}{\pi}\left\|F_{2}\right\|_{H_{2}}\left(\sup _{\lambda}\|\phi(\lambda+i \varepsilon)-g(\lambda)\|\right)^{2}\right)^{1 / 2} \\
& \times\left(\frac{b-a}{\pi}\left\|F_{1}\right\|_{H_{1}}\left(\sup _{\lambda}\|\psi(\lambda+i \varepsilon)\|\right)^{2}\right)^{1 / 2} \\
< & 2 \frac{b-a}{\pi} M\left\|F_{1}\right\|_{H_{1}}\left\|F_{2}\right\|_{H_{2}} \delta .
\end{aligned}
$$

Therefore, $\varepsilon<\gamma_{1}$ implies

$$
\begin{aligned}
|A(\varepsilon)+B(\varepsilon)+C(\varepsilon)| & <|A(\varepsilon)|+|B(\varepsilon)|+|C(\varepsilon)| \\
& \leqslant\left(1+4 \frac{b-a}{\pi} M\left\|F_{1}\right\|_{H_{1}}\left\|F_{2}\right\|_{H_{2}}\right) \delta .
\end{aligned}
$$

Thus, $\lim _{\varepsilon \downarrow 0} A(\varepsilon)+B(\varepsilon)+C(\varepsilon)=0$, which is the desired result.

REMARKS. (1) Let $\alpha$ and $\beta$ be channels. $T_{\alpha}$ and $T_{\beta}$ are multiplication operators in momentum representation, so they commute. Moreover, if $D(\alpha) \neq D(\beta)$ or $E_{\alpha} \neq$ $E_{\beta}$, then $T_{\alpha}-T_{\beta}$ has kernel $\{0\}$. To see this, notice that $T_{\alpha}$ and $T_{\beta}$ are multiplication by different polynomials in momentum representation (because $D(\alpha) \neq D(\beta)$ or $\left.E_{\alpha} \neq E_{\beta}\right)$. Therefore, $T_{\alpha}-T_{\beta}$ multiplies by a nonzero polynomial $Q\left(p_{1}, \ldots, p_{(N-1) m}\right)$. Functions in the kernel of $T_{\alpha}-T_{\beta}$ must have support in $\{p$ : $Q(p)=0\}$. This set is a nontrivial algebraic variety, and consequently has measure zero. Thus, $T_{\alpha}-T_{\beta}$ has kernel $\{0\}$.

(2) Suppose $\alpha$ is a channel with cluster decomposition $D$. Then $H_{D} P_{\alpha}=T_{\alpha} P_{\alpha}=$ $P_{\alpha} T_{\alpha}$ and $P_{\alpha}\left(z-H_{D}\right)^{-1}=P_{\alpha}\left(z-T_{\alpha}\right)^{-1}$. Also, if $P_{\alpha} F^{*}$ is Kato smooth with respect to $H_{D}$, then $P_{\alpha} F^{*}$ is Kato smooth with respect to $T_{\alpha}$.

(3) If $D$ is a cluster decomposition, then $P_{D}=\Sigma_{D(\alpha)=D} P_{\alpha}$. Consequently, $(z-$ $\left.H_{D}\right)^{-1} P_{D}=\Sigma_{D(\alpha)=D}\left(z-T_{\alpha}\right)^{-1} P_{\alpha}$. So, when the multiparticle limiting absorption principle holds for $H$,

$$
\begin{aligned}
(z-H)^{-1} & =\sum_{D}\left(z-H_{D}\right)^{-1} P_{D} \sum_{l=1}^{L(D)} F_{l, D} Z_{l, D}(z) \\
& =\sum_{\alpha}\left(z-T_{\alpha}\right)^{-1} P_{\alpha} \sum_{l=1}^{L(D(\alpha))} F_{l, D(\alpha)} Z_{l, D(\alpha)}(z)
\end{aligned}
$$

(4) In Propositions III.6 and III.7, we restrict some Fourier transforms to ellipsoids rather than spheres. This is because certain reduced masses are not equal. However, a linear change of coordinates shows that the results of $\$$ II.D apply to ellipsoids as well as spheres. 
The ellipsoids which we use are the surfaces in momentum space corresponding to a given energy for a channel Hamiltonian $T_{\alpha}$. In some clustered Jacobi coordinates, $T_{\alpha}=\sum_{l=1}^{k-1}\left(-2 M_{l}\right)^{-1} \Delta_{\zeta_{l}}+E_{\alpha}$, where $M_{l}$ is a reduced mass. We choose the "unit ellipsoid"

$$
\Gamma=\left\{q \in \mathbf{R}^{(k-1) m}: \sum_{l=1}^{k-1}\left(2 M_{l}\right)^{-1}\left|q_{l}\right|^{2}=1\right\}
$$

as the analogue of the unit sphere. The mapping $\pi_{\alpha}(k): L_{\delta}^{P} \otimes L^{2} \rightarrow L^{2}(\Gamma)$ is defined as the analogue of $\pi(k): L_{\delta}^{P} \otimes L^{2} \rightarrow L^{2}\left(S^{m-1}\right)$ from Proposition II.6.

(5) For convenience we identify the range of $P_{\alpha}$ with $\mathcal{H}_{\alpha}=\mathcal{H}(D(\alpha))$, defined in the introduction. The identification is given by the mapping $\phi \otimes \psi_{\alpha} \rightarrow \phi$, where $\phi \otimes \psi_{\alpha}$ denotes an arbitrary element of $P_{\alpha} \mathcal{H}$.

PROPOSITION III.6. Suppose the multiparticle limiting absorption principle holds for $H$, and let $\alpha$ be a channel. Let $\left(\zeta_{1}, \ldots, \zeta_{k-1}, \xi_{1}^{1}, \ldots, \xi_{n(k)-1}^{k}\right)$ be clustered Jacobi coordinates corresponding to the decomposition $D(\alpha)$, and let $\left(q_{1}, \ldots, q_{k-1}\right)$ be the Fourier conjugate variables to $\left(\zeta_{1}, \ldots, \zeta_{k-1}\right)$. Let $k_{\alpha}(q)=\left(\sum_{j=1}^{k-1}\left(2 M_{j}\right)^{-1}\left|q_{j}\right|^{2}\right)^{1 / 2}$, where $M_{j}$ is the reduced mass corresponding to the coordinate $\zeta_{j}$. Suppose the wave operators $\Omega_{\alpha}^{ \pm}$exist, and that $\phi \in L_{\delta_{0}}^{2}\left(\mathbf{R}^{(N-1) m}\right)$.

If $k^{2}+E_{\alpha}$ does not belong to the exceptional set $\mathcal{E}$ for $H$, then $\left(\left(\Omega_{\alpha}^{ \pm}\right)^{*} \phi\right)^{\wedge}$ may be restricted to the ellipsoid

$$
\Gamma(k)=\left\{q \in \mathbf{R}^{(k-1) m}: k_{\alpha}(q)=k\right\}
$$

and

$$
\pi_{\alpha}(k)\left(\Omega_{\alpha}^{ \pm}\right)^{*} \phi=\pi_{\alpha}(k) P_{\alpha} \sum_{l=1}^{L(D(\alpha))} F_{l, D(\alpha)} Z_{l, D(\alpha)}\left(k^{2}+E_{\alpha} \mp i 0\right) \phi .
$$

Proof. Choose an interval $[a, b]$ containing $k^{2}+E_{\alpha}$, but which does not intersect the exceptional set $\delta$. Choose $\psi_{1} \in \mathcal{H}_{\alpha}=\mathcal{H}(D(\alpha))$ so that $\psi_{1}$ is $C^{\infty}$ with support in $\cup_{\lambda \in[a, b]} \Gamma(\lambda)$.

Consider only $\Omega_{\alpha}^{-}$; the proof for $\Omega_{\alpha}^{+}$is similar.

The existence of $\Omega_{\alpha}^{-}$implies that $\Omega_{\alpha}^{-}$equals its Abelian limit (see [29]). So, if $\psi=\psi_{1} \otimes \psi_{\alpha}$, then

$$
\Omega_{\alpha}^{-} \psi=\lim _{\varepsilon \downarrow 0} 2 \varepsilon \int_{0}^{\infty} e^{-2 e t} e^{i t H} e^{-i t T \alpha} \psi d t .
$$

Thus, by Lemma II.1,

$$
\begin{aligned}
\left\langle\phi, \Omega_{\alpha}^{-} \psi\right\rangle & =\lim _{e \downarrow 0} 2 \varepsilon \int_{0}^{\infty}\left\langle e^{-e t} e^{-i t H} \phi, e^{-e t} e^{-i t T_{\alpha}} \psi\right\rangle d t \\
& =\lim _{\varepsilon \downarrow 0} \frac{\varepsilon}{\pi} \int_{-\infty}^{\infty}\left\langle(\lambda+i \varepsilon-H)^{-1} \phi,\left(\lambda+i \varepsilon-T_{\alpha}\right)^{-1} \psi\right\rangle d \lambda .
\end{aligned}
$$

Lemma III. 2 and the choice of $\psi_{1}$ now show

$$
\left\langle\phi, \Omega_{\alpha}^{-} \psi\right\rangle=\lim _{\varepsilon \downarrow 0} \frac{\varepsilon}{\pi} \int_{a}^{b}\left\langle(\lambda+i \varepsilon-H)^{-1} \phi,\left(\lambda+i \varepsilon-T_{\alpha}\right)^{-1} \psi\right\rangle d \lambda .
$$


By Remark (3) after Lemma III.5,

$$
(z-H)^{-1}=\sum_{\beta}\left(z-T_{\beta}\right)^{-1} P_{\beta} \sum_{l=1}^{L(D(\beta))} F_{l, D(\beta)} Z_{l, D(\beta)}(z)
$$

Hence,

$$
\begin{aligned}
\left\langle\phi, \Omega_{\alpha}^{-} \psi\right\rangle=\lim _{\varepsilon \downarrow 0} \frac{\varepsilon}{\pi} \int_{a}^{b}\left\langle\sum_{\beta} \sum_{l}\left(\lambda+i \varepsilon-T_{\beta}\right)^{-1} P_{\beta} F_{l, D(\beta)} Z_{l, D(\beta)}(z) \phi,\right. \\
\left.\left(\lambda+i \varepsilon-T_{\alpha}\right)^{-1} \psi\right\rangle d \lambda .
\end{aligned}
$$

By Lemma III.5 and Remark (1) after it, all terms with $D(\alpha) \neq D(\beta)$ or $E_{\alpha} \neq E_{\beta}$ vanish as $\varepsilon \rightarrow 0$. All other terms with $\alpha \neq \beta$ vanish because $P_{\beta} P_{\alpha}=0$ and $P_{\alpha} \psi=$

$$
\begin{aligned}
& \begin{array}{l}
\psi \text {. Therefore, } \\
\left\langle\phi, \Omega_{\alpha}^{-} \psi\right\rangle=\lim _{\varepsilon \downarrow 0} \frac{\varepsilon}{\pi} \int_{a}^{b}\left\langle\left(\lambda+i \varepsilon-T_{\alpha}\right)^{-1} P_{\alpha} \sum_{l=1}^{L(D(\alpha))} F_{l, D(\alpha)} Z_{l, D(\alpha)}(\lambda+i \varepsilon) \phi,\right. \\
\left.\left(\lambda+i \varepsilon-T_{\alpha}\right)^{-1} \psi\right\rangle d \lambda .
\end{array}
\end{aligned}
$$

For convenience put $F_{l}=F_{l, D(\alpha)}, L=L(D(\alpha))$, and $\phi_{l}(z)=Z_{l, D(\alpha)}(z) \phi$, so $\left\langle\phi, \Omega_{\alpha}^{-} \psi\right\rangle$

$$
=\sum_{l=1}^{L} \lim _{\varepsilon \downarrow 0} \frac{\varepsilon}{\pi} \int_{a}^{b}\left\langle\left(\lambda+i \varepsilon-T_{\alpha}\right)^{-1} P_{\alpha} F_{l} \phi_{l}(\lambda+i \varepsilon),\left(\lambda+i \varepsilon-T_{\alpha}\right)^{-1} \psi\right\rangle d \lambda .
$$

The identification of $P_{\alpha} \mathscr{H}$ with $\mathcal{H}_{\alpha}$ allows the inner product in this integral to be computed as an inner product on $\mathcal{H}_{\alpha}$. Moreover, as elements of $\mathcal{H}_{\alpha}, \psi_{1}$ and $P_{\alpha} F_{l} \phi_{l}(\lambda+i \varepsilon)$ have Fourier transforms which may be restricted to ellipsoids by Proposition II.6. So, using ellipsoidal coordinates on $\mathbf{R}^{(k-1) m}$ to compute the $\mathcal{H}_{\alpha}$ inner product, we have

$$
\begin{aligned}
\left\langle\phi, \Omega_{\alpha}^{-} \psi\right\rangle=\sum_{l=1}^{L} \lim _{\varepsilon \downarrow 0} \int_{a}^{b} \int_{0}^{\infty} \frac{\varepsilon / \pi}{\left(k^{2}+E_{\alpha}-\lambda\right)^{2}+\varepsilon^{2}} \\
\times\left\langle\pi_{\alpha}(k) P_{\alpha} F_{l} \phi_{l}(\lambda+i \varepsilon), \pi_{\alpha}(k) \psi_{1}\right\rangle_{L^{2}(\Gamma)} d k d \lambda,
\end{aligned}
$$

where $\pi_{\alpha}(k): \mathcal{H}_{\alpha} \rightarrow L^{2}(\Gamma)$ restricts Fourier transforms to $\Gamma(k)$, and then does the scaling to yield a function on $\Gamma=\Gamma(1)$.

The inner product in the last integrand depends continuously on $k$ and has support in $[a, b]$ in the $k$ variable. Thus, Fubini's theorem and the dominated convergence theorem may be applied to yield:

$$
\begin{aligned}
\left\langle\phi, \Omega_{\alpha}^{-} \psi\right\rangle=\sum_{l=1}^{L} \int_{a}^{b} \lim _{\varepsilon \downarrow 0} \int_{a}^{b} \frac{\varepsilon / \pi}{\left(k^{2}+E_{\alpha}-\lambda\right)^{2}+\varepsilon^{2}} \\
\quad \times\left\langle\pi_{\alpha}(k) P_{\alpha} F_{l} \phi_{l}(\lambda+i \varepsilon), \pi_{\alpha}(k) \psi_{1}\right\rangle_{L^{2}(\Gamma)} d \lambda d k .
\end{aligned}
$$

The limit may now be computed explicitly (Ikebe [12], Simon [29]):

$$
\left\langle\phi, \Omega_{\alpha}^{-} \psi\right\rangle=\int_{a}^{b}\left\langle\pi_{\alpha}(k) P_{\alpha} \sum_{l} F_{l} \phi_{l}\left(k^{2}+E_{\alpha}+i 0\right), \pi_{\alpha}(k) \psi_{1}\right\rangle_{L^{2}(\Gamma)} d k .
$$

Since $\psi_{1}$ is an arbitrary element of a dense subspace of $E^{\alpha}([a, b]) \mathcal{H}_{\alpha}$, the proposition follows. 
Proposition III.7. Suppose the multiparticle limiting absorption principle holds for $H$. Suppose $\Omega_{\alpha}^{ \pm}$exist. Let $[a, b]$ be any compact interval disjoint from the exceptional set $\mathcal{E}$. Let $E[a, b]$ be the spectral projection for $H$ on the interval $[a, b]$. Then, $\phi \in L_{\delta_{0}}^{2}\left(\mathbf{R}^{(N-1) m}\right)$ implies

$$
\|E[a, b] \phi\|^{2}=\sum_{\alpha} \int_{a<k_{\alpha}(q)+E_{\alpha}<b}\left|\left(\Omega_{\alpha}^{ \pm *} \phi\right)^{\wedge}(q)\right|^{2} d q .
$$

Proof. Apply Stone's formula and the first resolvent formula:

$$
\|E[a, b] \phi\|^{2}=\lim _{\varepsilon \downarrow 0} \frac{\varepsilon}{\pi} \int_{a}^{b}\left\langle(\lambda \pm i \varepsilon-H)^{-1} \phi,(\lambda \pm i \varepsilon-H)^{-1} \phi\right\rangle d \lambda .
$$

For simplicity take only the + sign (this yields the result for $\Omega^{-}$). Then use the multiparticle limiting absorption principle formula:

$$
\begin{aligned}
\|E[a, b] \phi\|^{2}= & \sum_{\alpha, \beta} \sum_{l=1}^{L(D(\alpha))} \sum_{j=1}^{L(D(\beta))} \lim _{\varepsilon \downarrow 0} \frac{\varepsilon}{\pi} \\
& \times \int_{a}^{b}\left\langle\left(\lambda+i \varepsilon-T_{\alpha}\right)^{-1} P_{\alpha} F_{l, D(\alpha)} \phi_{l, D(\alpha)}(\lambda+i \varepsilon),\right. \\
& \left.\left(\lambda+i \varepsilon-T_{\beta}\right)^{-1} P_{\beta} F_{j, D(\beta)} \phi_{j, D(\beta)}(\lambda+i \varepsilon)\right\rangle d \lambda,
\end{aligned}
$$

where $\phi_{l, D(\alpha)}(z)=Z_{l, D(\alpha)}(z) \phi$

By Lemma III.5 and Remark (1) after it, all terms with $D(\alpha) \neq D(\beta)$ or $E_{\alpha} \neq E_{\beta}$ vanish as $\varepsilon \rightarrow 0$. All other terms with $\alpha \neq \beta$ vanish because $P_{\alpha} P_{\beta}=0$. Therefore,

$$
\|E[a, b] \phi\|^{2}=\sum_{\alpha} \lim _{\varepsilon \downarrow 0} \int_{a}^{b} \frac{\varepsilon}{\pi}\left\|\sum_{l=1}^{L}\left(\lambda+i \varepsilon-T_{\alpha}\right)^{-1} P_{\alpha} F_{l, D(\alpha)} \phi_{l, D(\alpha)}(\lambda+i \varepsilon)\right\|^{2} d \lambda .
$$

Proposition II. 2 and the dominated convergence theorem show that the limit may be taken through the integral:

$$
\begin{aligned}
& \|E[a, b] \phi\|^{2}=\sum_{\alpha} \int_{a}^{b}(2 \pi i)^{-1} \sum_{l} \sum_{j} \\
& \quad\left[\left\langle\phi_{l, D(\alpha)}(\lambda+i 0),\left(F_{l, D(\alpha)}^{*}\left(\lambda-i 0-T_{\alpha}\right)^{-1} P_{\alpha} F_{j, D(\alpha)}\right) \phi_{j, D(\alpha)}(\lambda+i 0)\right\rangle\right. \\
& \left.\quad-\left\langle\phi_{l, D(\alpha)}(\lambda+i 0),\left(F_{l, D(\alpha)}^{*}\left(\lambda+i 0-T_{\alpha}\right)^{-1} P_{\alpha} F_{j, D(\alpha)}\right) \phi_{j, D(\alpha)}(\lambda+i 0)\right\rangle\right] d \lambda .
\end{aligned}
$$

Lemma II.4 shows that this may be rewritten as

$$
\begin{aligned}
& \|E[a, b] \phi\|^{2} \\
& \quad=\sum_{\alpha} \int_{a}^{b} \lim _{\varepsilon \downarrow 0} \frac{\varepsilon}{\pi}\left\|\left(\lambda+i \varepsilon-T_{\alpha}\right)^{-1} E^{\alpha}[a, b] P_{\alpha} \sum_{l} F_{l, D(\alpha)} \phi_{l, D(\alpha)}(\lambda+i \varepsilon)\right\|^{2} d \lambda .
\end{aligned}
$$

The quantity inside the norm in the integrand belongs to $\operatorname{Ran} P_{\alpha}$, so it may be lifted to $\mathcal{H}_{\alpha}$ by the identification of $\mathcal{H}_{\alpha}$ with $P_{\alpha} \mathcal{H}$. Ellipsoidal coordinates may then be used to evaluate the norm on $\mathcal{F}_{\alpha}$ : 


$$
\begin{aligned}
\|E[a, b] \phi\|^{2}=\sum_{\alpha} \int_{a}^{b} \lim _{\varepsilon \downarrow 0} \int_{\left(a-E_{\alpha}\right)^{1 / 2}}^{\left(b-E_{\alpha}\right)^{1 / 2}} \frac{\varepsilon / \pi}{\left(k^{2}+E_{\alpha}-\lambda\right)^{2}+\varepsilon^{2}} \\
\times\left\|\pi_{\alpha}(k) P_{\alpha} \sum_{l} F_{l, D(\alpha)} \phi_{l, D(\alpha)}(\lambda+i \varepsilon)\right\|_{L^{2}(\Gamma)}^{2} d k d \lambda .
\end{aligned}
$$

The limit may be evaluated explicitly (Ikebe [12], Simon [29]). Proposition III.6 and a change of variables yield the desired result:

$$
\begin{aligned}
\|E[a, b] \phi\|^{2} & =\sum_{\alpha} \int_{a}^{b}\left\|\pi_{\alpha}(\nu) P_{\alpha} \sum_{l} F_{l, D(\alpha)} \phi_{l, D(\alpha)}\left(\nu^{2}+E_{\alpha}+i 0\right)\right\|_{L^{2}(\Gamma)}^{2} d \nu \\
& =\sum_{\alpha} \int_{a<k_{\alpha}(q)^{2}+E_{\alpha}<b}\left|\left(\Omega_{\alpha}^{-*} \phi\right)^{\wedge}(q)\right|^{2} d q .
\end{aligned}
$$

Proof of Theorem III.1. The wave operators are partial isometries, so it suffices to prove

$$
\left\|P_{\text {a.c. }} \phi\right\|^{2}=\sum_{\alpha}\left\|\Omega_{\alpha}^{ \pm *} \phi\right\|^{2}
$$

for $\phi \in L_{\delta_{0}}^{2}\left(\mathbf{R}^{(N-1) m}\right)$.

The complement of the exceptional set $\mathcal{E}$ is open, so it is the countable union of compact intervals $A_{n}$, with rational end points, such that any two distinct intervals intersect in at most one point. Since $\mathcal{E}$ has measure zero,

$$
\left\|P_{a c} \phi\right\|^{2}=\sum_{n}\left\|E\left(A_{n}\right) \phi\right\|^{2}
$$

where $E\left(A_{n}\right)$ is the spectral projection for $H$ corresponding to the interval $A_{n}$.

Therefore, by Proposition III.7 and Fubini's theorem,

$$
\begin{aligned}
\left\|P_{\text {a.c. }} \phi\right\|^{2} & =\sum_{n=1}^{\infty} \sum_{\alpha} \int_{k_{\alpha}(q)^{2}+E_{\alpha} \in A_{n}}\left|\left(\Omega_{\alpha}^{ \pm *} \phi\right)^{\wedge}(q)\right|^{2} d q \\
& =\sum_{\alpha}\left\|\left(\Omega_{\alpha}^{ \pm *} \phi\right)^{\wedge}\right\|^{2}=\sum_{\alpha}\left\|\Omega_{\alpha}^{ \pm *} \phi\right\|^{2} .
\end{aligned}
$$

IV. Resolvent formulas. Having proved Theorem III.1, the problem of asymptotic completeness is reduced to the study of resolvent formulas which satisfy certain conditions. Derivation of such formulas for the two, three, and four particle cases is the goal of this section.

To begin with, the desired two-body formula is given in equation (IV.1). Next, a generalization of equation (IV.1) is obtained for the $N$-particle case. This is equation (IV.4), which involves $\frac{1}{2} N(N-1) \times \frac{1}{2} N(N-1)$ matrices. It coincides with the Faddeev equation [6] when $N=3$, but does not have the form required by the multiparticle limiting absorption principle. Thus, various modifications are necessary. 
A second $N$-body formula, equation (IV.5), is obtained from equation (IV.4), by increasing the size of the matrices so that the individual matrix entries become simpler. This formula is closely related, but not identical to a formula of Narodetskii and Yakubovskii [20]. However, as was the case with the first $N$-body formula, equation (IV.5) is not in a suitable form for scattering.

Consequently, for $N=3$ and $N=4$, a third formula, which does have the correct form, is derived from equation (IV.5). Ginibre and Moulin [7] have used this three-body equation, and their ideas have been generalized to give the four-body formula.

For $N>5$ analogous equations, having the proper form, may be written down. However, it is not at all clear that the bounds required by the multiparticle limiting absorption principle can be proved. This is due to the fact that we have not been able to prove the absence of spurious zeros in the four-body case.

Throughout this section we assume that $V_{i j}=U_{i j} W_{i j}$, such that $U_{i j}\left(-\Delta_{i j}+\right.$ $1)^{-1 / 2}$ and $W_{i j}\left(-\Delta_{i j}+1\right)^{-1 / 2}$ are compact as operators on $L^{2}\left(\mathbf{R}^{m}\right)$, where $\Delta_{i j}$ is the Laplacian in the $x_{i j}$ variable. These assumptions imply the relative form boundedness of the potentials with respect to $H_{0}$, with relative bound zero. So, when $\operatorname{Re} z$ is sufficiently large negative, the perturbation series

$$
\begin{aligned}
(z-H)^{-1}= & \left(z-H_{0}\right)^{-1}+\left(z-H_{0}\right)^{-1} \sum_{\alpha} V_{\alpha}\left(z-H_{0}\right)^{-1} \\
& +\left(z-H_{0}\right)^{-1} \sum_{\alpha} V_{\alpha}\left(z-H_{0}\right)^{-1} \sum_{\beta} V_{\beta}\left(z-H_{0}\right)^{-1}+\ldots
\end{aligned}
$$

is norm convergent.

When $N=2$, this is particularly simple. Using geometric series, the perturbation expansion may be resummed in the form:

$$
\begin{aligned}
(z-H)^{-1}= & \left(z-H_{0}\right)^{-1} \\
& +\left(z-H_{0}\right)^{-1} U\left[1-W\left(z-H_{0}\right)^{-1} U\right]^{-1} W\left(z-H_{0}\right)^{-1}
\end{aligned}
$$

This is the desired equation when $N=2$.

For $N>3$, the graphical symbolism of Weinberg [40] (see also Simon [29]) is used to identify each term of the perturbation series with a graph. For example, when $N=4$, the term

$$
\left(z-H_{0}\right)^{-1} V_{12}\left(z-H_{0}\right)^{-1} V_{23}\left(z-H_{0}\right)^{-1} V_{12}\left(z-H_{0}\right)^{-1} V_{24}\left(z-H_{0}\right)^{-1}
$$

is assigned the graph

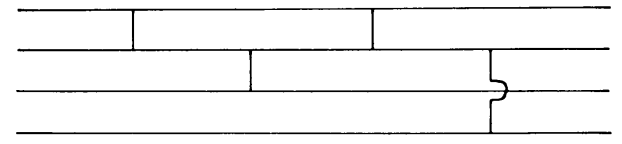


Using this symbolism, the sum of all nontrivial graphs is

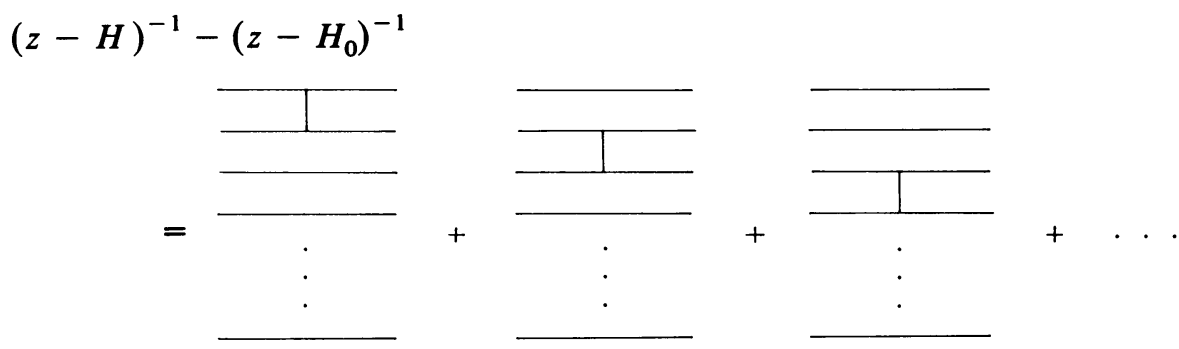

Several definitions are now required:

DEFINITION. To each nontrivial graph

$$
\left(z-H_{0}\right)^{-1} V_{\alpha_{1}}\left(z-H_{0}\right)^{-1} V_{\alpha_{2}} \cdots\left(z-H_{0}\right)^{-1} V_{\alpha_{n}}\left(z-H_{0}\right)^{-1},
$$

there is associated a truncated graph

$$
W_{\alpha_{1}}\left(z-H_{0}\right)^{-1} V_{\alpha_{2}} \cdots\left(z-H_{0}\right)^{-1} V_{\alpha_{n}}\left(z-H_{0}\right)^{-1} .
$$

A truncated graph is connected (respectively disconnected) if its associated graph is connected (disconnected).

Definition. An expression of the form

$$
W_{\alpha_{1}}\left(z-H_{0}\right)^{-1} V_{\alpha_{2}}\left(z-H_{0}\right)^{-1} \cdots\left(z-H_{0}\right)^{-1} V_{\alpha_{n-1}}\left(z-H_{0}\right)^{-1} U_{\alpha_{n}}
$$

is a barely connected part if

$$
W_{\alpha_{1}}\left(z-H_{0}\right)^{-1} V_{\alpha_{2}}\left(z-H_{0}\right)^{-1} \cdots\left(z-H_{0}\right)^{-1} V_{\alpha_{n-1}}\left(z-H_{0}\right)^{-1} V_{\alpha_{n}}\left(z-H_{0}\right)^{-1}
$$

is connected, but

$$
W_{\alpha_{1}}\left(z-H_{0}\right)^{-1} V_{\alpha_{2}}\left(z-H_{0}\right)^{-1} \cdots\left(z-H_{0}\right)^{-1} V_{\alpha_{n-1}}\left(z-H_{0}\right)^{-1}
$$

is disconnected.

Definition. $Q_{\alpha}(z)=\left(z-H_{0}\right)^{-1} U_{\alpha}$.

$\mathfrak{T}_{\alpha, \beta}(z)$ is the sum of all barely connected parts whose initial factor is $W_{\alpha}$ and whose final factor is $U_{\beta}$.

$\bigodot_{\alpha}(z)$ is the sum of all disconnected truncated graphs whose initial factor is $W_{\alpha}$.

Let $G=W_{\alpha_{1}}\left(z-H_{0}\right)^{-1} V_{\alpha_{2}}\left(z-H_{0}\right)^{-1} \cdots V_{\alpha_{n}}\left(z-H_{0}\right)^{-1}$ be a truncated graph. Either $G$ is disconnected or $G$ is the product of a barely connected part $W_{\alpha_{1}}(z-$ $\left.H_{0}\right)^{-1} V_{\alpha_{2}}\left(z-H_{0}\right)^{-1} \cdots\left(z-H_{0}\right)^{-1} U_{\alpha_{j}}$ and a truncated graph $G^{\prime}=W_{\alpha_{1}}(z-$ $\left.H_{0}\right)^{-1} V_{\alpha_{j+1}}\left(z-H_{0}\right)^{-1} \cdots V_{\alpha_{n}}\left(z-H_{0}\right)^{-1}$. By iterating this procedure $G$ may be uniquely decomposed as a product of $k \geqslant 0$ barely connected parts times a disconnected truncated graph.

In order to compute the sum of all graphs, first sum all graphs containing precisely $k$ barely connected parts, and then sum over $k$. The sum of all nontrivial graphs with no barely connected parts is the sum of all nontrivial disconnected graphs: $\Sigma_{\alpha} \mathbb{Q}_{\alpha} \mathcal{C}_{\alpha}$. The sum of all graphs containing one barely connected part is $\Sigma_{\alpha, \beta} \mathcal{Q}_{\alpha} \mathfrak{T}_{\alpha, \beta} \mathcal{C}_{\beta}$. Similarly, the sum of all graphs containing $k$ barely connected parts is

$$
\sum_{\alpha, \beta_{1}, \beta_{2}, \ldots, \beta_{k}} \mathbb{Q}_{\alpha} \mathfrak{R}_{\alpha, \beta_{1}} \mathfrak{T}_{\beta_{1}, \beta_{2}} \mathfrak{T}_{\beta_{2}, \beta_{3}} \cdots \mathfrak{T}_{\beta_{k-1}, \beta_{k}} \mathcal{C}_{\beta_{k}} .
$$


Therefore, using matrix multiplication, $(z-H)^{-1}-\left(z-H_{0}\right)^{-1}=\mathbb{Q}(1+\Re+$ $\left.\mathfrak{N}^{2}+\ldots\right)$.

When $\operatorname{Re} z$ is sufficiently large negative, the geometric series $1+\mathfrak{K}(z)+$ $\mathscr{T}(z)^{2}+\ldots$ converges in norm to $(1-\mathscr{R}(z))^{-1}$ and

$$
(z-H)^{-1}=\left(z-H_{0}\right)^{-1}+\mathbb{Q}(z)(1-\mathfrak{N}(z))^{-1} \mathcal{C}(z) \text {. }
$$

To obtain explicit formulas for $\mathscr{R}(z)$ and $\mathcal{C}(z)$ in terms of the resolvents $\left(z-H_{D}\right)^{-1}$, it is useful to introduce strings and make several definitions.

Definition. Suppose $2 \leqslant k \leqslant N-1$. A string is an ordered collection of cluster decompositions $S=\left(D_{N}, D_{N-1}, \ldots, D_{k}\right)$, such that

(i) $D_{l}$ has $l$ clusters, and

(ii) $D_{l+1}$ is a refinement of $D_{l}$, i.e., $D_{l+1}$ is formed from $D_{l}$ by splitting one cluster into two.

Definition. A string $S=\left(D_{N}, D_{N-1}, \ldots, D_{k}\right)$ is called long if $k=2$. If $k \geqslant 3$, then $S$ is a short string.

Definition. Suppose $S=\left(D_{N}, D_{N-1}, \ldots, D_{k}\right)$ is a string. Since $D_{N-1}$ has exactly one cluster containing two elements, it may be identified with a pair $\alpha$. Define $U_{S}=U_{\alpha}$ and

$$
\begin{aligned}
\tilde{C}_{S}(z)= & W_{\alpha}\left(z-H_{D_{N-1}}\right)^{-1}\left(V_{D_{N-2}}-V_{D_{N-1}}\right)\left(z-H_{D_{N-2}}\right)^{-1} \\
& \cdots\left(V_{D_{k}}-V_{D_{k-1}}\right)\left(z-H_{D_{k}}\right)^{-1}
\end{aligned}
$$

Definition. Let $S=\left(D_{N}, D_{N-1}, \ldots, D_{k}\right)$ and $S^{\prime}=\left(D_{N}^{\prime}, D_{N-1}^{\prime}, \ldots, D_{l}^{\prime}\right)$ be strings.

$$
\tilde{M}_{S, S^{\prime}}(z)= \begin{cases}0 & \text { if } S \text { is short, } \\ 0 & \text { if } S \text { is long and } D_{N-1}^{\prime} \text { refines } D_{2}, \\ \tilde{C}_{S} U_{S^{\prime}} & \text { if } S \text { is long and } D_{N-1}^{\prime} \text { does not refine } D_{2} .\end{cases}
$$

REMARK. $\tilde{M}_{S, S^{\prime}}$ has been defined so that its nonzero entries are sums of barely connected parts. The partition $D_{N-1}^{\prime}$ refines $D_{2}$ if and only if $\tilde{C}_{S} U_{S^{\prime}}$ is not a sum of barely connected parts.

Each disconnected truncated graph

$$
G=W_{\alpha_{1}}\left(z-H_{0}\right)^{-1} V_{\alpha_{2}}\left(z-H_{0}\right)^{-1} \ldots V_{\alpha_{n}}\left(z-H_{0}\right)^{-1}
$$

has an associated string determined by the following procedure. For $j=$ $1,2, \ldots, n$, there is a unique cluster decomposition $D(j)$, with a maximal number of clusters, such that all the pairs $\alpha_{1}, \ldots, \alpha_{j}$ refine $D(j)$. Since $G$ is disconnected, the sequence $D(1), D(2), \ldots, D(k)$ has the following properties:

(i) $D(1)$ has $N-1$ clusters,

(ii) $D(n)$ has $k \geqslant 2$ clusters,

(iii) either $D(i)=D(i+1)$ or $D(i)$ has one cluster more than $D(i+1)$, and

(iv) $D(i)$ refines $D(j)$ whenever $i \leqslant j$.

As a consequence, removal of the repetitions in the sequence $D(1), D(2), \ldots, D(n)$ yields a sequence $D_{N-1}, D_{N-2}, \ldots, D_{k}$. The string associated with $G$ is $S=$ $\left(D_{N}, D_{N-1}, \ldots, D_{k}\right)$. 
At large negative $\operatorname{Re} z$, each resolvent $\left(z-H_{D}\right)^{-1}$ equals its perturbation series. Substituting the series for each resolvent factor in $\tilde{C}_{S}(z), \tilde{C}_{S}(z)$ becomes the sum of all truncated graphs whose string is $S$. Thus $\mathcal{C}_{\alpha}(z)$ may be written as the sum of all $\tilde{C}_{S}(z)$, such that $S=\left(D_{N}, D_{N-1}, \ldots, D_{k}\right)$, and $D_{N-1}$ is the decomposition determined by the pair $\alpha$.

Similarly, $\mathfrak{T}_{\alpha, \beta}(z)$ is the sum of terms $\tilde{M}_{S, S^{\prime}}(z)$, where $S^{\prime}$ is any string with $D_{N-1}^{\prime}$ identified with $\beta$, and the sum is over all $S$, with $D_{N-1}$ identified with $\alpha$.

With $\mathfrak{N}$ and $\mathcal{C}$ written in closed form, we have

$$
(z-H)^{-1}=\left(z-H_{0}\right)^{-1}+\mathscr{Q}(z)(1-\mathscr{T}(z))^{-1} \mathcal{C}(z) \text {. }
$$

By the quadratic form methods of $\S I I B, \mathcal{Q}(z), \mathscr{N}(z)$, and $\mathcal{C}(z)$ are analytic for $z \in \mathbf{C} \backslash \sigma_{\text {ess }}(H)$, and $\mathscr{R}(z)$ is compact. Therefore, the uniqueness of analytic continuation shows that equation (IV.4) is valid for $z \in C \backslash \sigma(H)$, except for an at most countable set, where the right-hand side may have removable singularities.

EXAMPLES. (1) When $N=3$, equation (IV.4) is a modified form of the Faddeev equation [6]. This equation with $U_{\alpha}=\left|V_{\alpha}\right|^{1 / 2}$ and $W_{\alpha}=\left|V_{\alpha}\right|^{1 / 2} \operatorname{sgn}\left(V_{\alpha}\right)$ may also be found in Ginibre and Moulin [7]. Explicitly

$$
\begin{aligned}
\mathcal{Q}(z) & =\left[\begin{array}{lcc}
\left(z-H_{0}\right)^{-1} U_{12} & \left(z-H_{0}\right)^{-1} U_{13} & \left(z-H_{0}\right)^{-1} U_{23}
\end{array}\right] \\
\mathscr{N}(z) & =\left[\begin{array}{ccc}
0 & W_{12}\left(z-H_{12}\right)^{-1} U_{13} & W_{12}\left(z-H_{12}\right)^{-1} U_{23} \\
W_{13}\left(z-H_{13}\right)^{-1} U_{12} & 0 & W_{13}\left(z-H_{13}\right)^{-1} U_{23} \\
W_{23}\left(z-H_{23}\right)^{-1} U_{12} & W_{23}\left(z-H_{23}\right)^{-1} U_{13} & 0
\end{array}\right] \\
\mathcal{C}(z) & =\left[\begin{array}{l}
W_{12}\left(z-H_{12}\right)^{-1} \\
W_{13}\left(z-H_{13}\right)^{-1} \\
W_{23}\left(z-H_{23}\right)^{-1}
\end{array}\right] .
\end{aligned}
$$

(2) When $N=4$, the matrices have six rows or columns, and the entries are more complicated. The following is a representative sample of the entries:

$$
\begin{aligned}
\mathcal{Q}_{12}(z)= & \left(z-H_{0}\right)^{-1} U_{12} \\
\mathfrak{T}_{12,12}(z)= & 0 \\
\mathfrak{R}_{12,13}(z)= & W_{12}\left(z-H_{12}\right)^{-1}\left(V_{14}+V_{24}\right)\left(z-H_{124}\right)^{-1} U_{13} \\
& +W_{12}\left(z-H_{12}\right)^{-1} V_{34}\left(z-H_{12,34}\right)^{-1} U_{13} \\
\mathfrak{R}_{12,34}(z)= & W_{12}\left(z-H_{12}\right)^{-1}\left(V_{13}+V_{23}\right)\left(z-H_{123}\right)^{-1} U_{34} \\
& +W_{12}\left(z-H_{12}\right)^{-1}\left(V_{14}+V_{24}\right)\left(z-H_{124}\right)^{-1} U_{34}, \\
\mathcal{C}_{12}(z)= & W_{12}\left(z-H_{12}\right)^{-1}+W_{12}\left(z-H_{12}\right)^{-1}\left(V_{13}+V_{23}\right)\left(z-H_{123}\right)^{-1} \\
& +W_{12}\left(z-H_{12}\right)^{-1}\left(V_{14}+V_{24}\right)\left(z-H_{124}\right)^{-1} \\
& +W_{12}\left(z-H_{12}\right)^{-1} V_{34}\left(z-H_{12,34}\right)^{-1}
\end{aligned}
$$


For $N \geqslant 4$, several of the matrix entries appearing in equation (IV.4) contain many terms. In order to avoid certain combinatorial difficulties it is necessary to obtain matrices with less complicted entries. This can be done at the expense of using larger matrices.

Fix $N \geqslant 3$ and let $l$ denote the number of strings which occur in the $N$ body case. $\tilde{A}(z)$ is the $1 \times l$ matrix with $\tilde{A}_{S}(z)=\left(z-H_{0}\right)^{-1} U_{S} \cdot \tilde{M}_{S, S^{\prime}}(z)$ is the $l \times l$ matrix whose entries are given by equation (IV.3). $\tilde{C}(z)$ is the $l \times 1$ matrix whose entries are given by equation (IV.2).

Our second formula for the $N$-body resolvent is

$$
(z-H)^{-1}=\left(z-H_{0}\right)^{-1}+\tilde{A}(z)(1-\tilde{M}(z))^{-1} \tilde{C}(z) .
$$

The validity of this formula may be established by computing $\mathscr{Q}(z)(\mathscr{T}(z))^{k} \mathcal{C}(z)$, with the entries of $\Re(z)$ expressed in terms of the operators $\tilde{M}_{S, S^{\prime}}(z)$. The result of this computation is $\tilde{A}(z)(\tilde{M}(z))^{k} \tilde{C}(z)$. Equation (IV.5) then follows from equation (IV.4) by using geometric series.

An alternative proof may be given. Notice first that each barely connected part is the product of a disconnected truncated graph and a final factor $U_{\beta}$. Therefore, each barely connected part has an associated string determined by its initial truncated graph.

Next, recall that each graph $G$ may be uniquely decomposed as $A_{\alpha} B_{1} B_{2} \ldots B_{k} G^{\prime}$, where $B_{1}, B_{2}, \ldots, B_{k}$ are barely connected parts and $G^{\prime}$ is disconnected. Let $S_{1}, S_{2}, \ldots, S_{k+1}$ be the strings associated to $B_{1}, B_{2}, \ldots, B_{k}$, and $G^{\prime}$, respectively. Then $G$ determines the sequence $S_{1}, S_{2}, \ldots, S_{k+1}$ uniquely.

To sum all graphs, first sum all graphs with a given sequence of strings, and then sum over all sequences. Since $\tilde{M}_{S, S^{\prime}}(z)$ is the sum of all barely connected parts with string $S$ and final factor $U_{S^{\prime}}$, the sum of all graphs with sequence $S_{1}, S_{2}, \ldots, S_{k+1}$ is: $\tilde{A}_{S_{1}} \tilde{M}_{S_{1}, S_{2}} \tilde{M}_{S_{2}, S_{3}} \ldots \tilde{M}_{S_{k}, S_{k \neq 1}} \tilde{C}_{S_{k+1}}$. Thus, the sum of all graphs with sequence of length $k+1$ is $\tilde{A}(z)(\tilde{M}(z))^{k} \tilde{C}(z)$. Equation (IV.5) follows by using geometric series and the uniqueness of analytic continuation.

REMARK. Equation (IV.4) involves a $\frac{1}{2} N(N-1) \times \frac{1}{2} N(N-1)$ matrix in the $N$ particle case. Equation (IV.5) contains a much larger matrix. For the $N$ particle case, it is $l_{N} \times l_{N}$, where $l_{3}=3$ and $l_{N}=\frac{1}{2} N(N-1)\left(l_{N-1}+1\right)$.

$$
\begin{array}{ll}
N=3: & l_{N}=3, \\
N=4: & l_{N}=24, \\
N=5: & l_{N}=250, \\
N=6: & l_{N}=3765 .
\end{array}
$$

Next, we give the final resolvent formula for the three body case. The same formula may be found in Ginibre and Moulin [7]; similar equations may be found in Thomas [36] and Howland [11].

For the moment, the operators $\rho_{12}, \rho_{13}$, and $\rho_{23}$ will not be specified. They will be chosen in $\S \mathrm{V}$ so that certain bounds can be established. Algebraically, equation (IV.6) holds for any choice of these $\rho$ functions. 
Denoting $\left(z-H_{0}\right)^{-1}$ and $\left(z-H_{\alpha}\right)^{-1}$ by $G_{0}$ and $G_{\alpha}$, respectively, the final three-body equation is:

$$
(z-H)^{-1}=\left(z-H_{0}\right)^{-1}+A(z)(1-M(z))^{-1} C(z),
$$

where the matrices $A(z), M(z)$ and $C(z)$ are given in Figure 1 .

We view $A(z), M(z)$, and $C(z)$ as $1 \times 3,3 \times 3$, and $3 \times 1$ matrices of $1 \times 2$, $2 \times 2$, and $2 \times 1$ blocks, respectively. In accordance with this viewpoint, we label the entries $A_{\alpha, i}, M_{\alpha, i ; \beta, j}$, and $C_{\alpha, i}$, where $\alpha$ and $\beta$ run over the pairs, while $i$ and $j$ run from 1 to 2 .

Equation (IV.6) holds because

$$
A(z)(M(z))^{k} C(z)=\mathbb{Q}(z)(\mathscr{N}(z))^{k} \mathcal{C}(z)
$$

for $k \geqslant 0$, and because geometric series may be used at large negative $\operatorname{Re} z$ when the $\rho_{\alpha}$ are suitably chosen.

To prove equation (IV.7), consider a typical nonzero term in the expression for $Q \mathbb{T}^{k} \mathcal{C}$. It has the form

$$
\begin{aligned}
\mathbb{Q}_{\alpha_{1}} \mathfrak{T}_{\alpha_{1}, \alpha_{2}} & \ldots \Re_{\alpha_{k}, \alpha_{k+1}} \mathcal{C}_{\alpha_{k+1}} \\
& =G_{0} U_{\alpha_{1}} W_{\alpha_{1}} G_{\alpha_{1}} U_{\alpha_{2}} W_{\alpha_{2}} G_{\alpha_{2}} U_{\alpha_{3}} \ldots W_{\alpha_{k}} G_{\alpha_{k}} U_{\alpha_{k+1}} W_{\alpha_{k+1}} G_{\alpha_{k+1}},
\end{aligned}
$$

where $\alpha_{i} \neq \alpha_{i+1}$.

Let $Q_{\alpha, 1}=\left(1-P_{\alpha}\right)$ and $Q_{\alpha, 2}=P_{\alpha}=\left(P_{\alpha} \rho_{\alpha}\right)\left(\rho_{\alpha}^{-1} P_{\alpha}\right)$, and replace each $G_{\alpha}$ factor by $G_{\alpha} Q_{\alpha, 1}+G_{\alpha} Q_{\alpha, 2}$ in equation (IV.8). The right side of equation (IV.8) becomes a sum of $2^{k+1}$ terms of the form:

$$
\begin{gathered}
G_{0} U_{\alpha_{1}} W_{\alpha_{1}} G_{\alpha_{1}} Q_{\alpha_{1}, i_{1}} U_{\alpha_{2}} W_{\alpha_{2}} G_{\alpha_{2}} Q_{\alpha_{2}, i_{2}} U_{\alpha_{3}} \ldots W_{\alpha_{k+1}} G_{\alpha_{k+1}} Q_{\alpha_{k+1}, i_{k+1}} \\
=A_{\alpha_{1}, i_{1}} M_{\alpha_{1}, i_{1} ; \alpha_{2}, i_{2}} M_{\alpha_{2}, i_{2} ; \alpha_{3}, i_{3}} \ldots M_{\alpha_{k}, i_{k} ; \alpha_{k+1}, i_{k+1}} C_{\alpha_{k+1}, i_{k+1}}
\end{gathered}
$$

Summing over all indices and using the identity $G_{0} V_{\alpha} G_{\alpha}=\left(G_{\alpha}-G_{0}\right)$, equation (IV.7) is obtained.

Equations (IV.4) and (IV.5) are not suitable for scattering because $\mathfrak{R}(z)$, $\mathcal{C}(z), \tilde{M}(z)$, and $\tilde{C}(z)$ are all singular as $z$ approaches the essential spectrum of $H$. The advantage of equation (IV.6) is that $M(z)$ and $C(z)$ are reasonably well behaved as $z$ approaches the essential spectrum, and the singularities all appear explicitly in the factor $A(z)$.

Equation (IV.6) was obtained from equation (IV.4) by the following procedure: First, the inverse term in equation (IV.4) was expanded using geometric series. Second, the factors $\left(1-P_{\alpha}\right)$ and $P_{\alpha}=P_{\alpha} \rho_{\alpha} \rho_{\alpha}^{-1} P_{\alpha}$ were inserted after each $G_{\alpha}$. Third, each term was decomposed as a product in a way which depended on the sequence of projections $\left(1-P_{\alpha}\right)$ or $P_{\alpha}$. Then, the result was resummed to give equation (IV.6).

The four body case is similar. Equations (IV.4) and (IV.5) are not suitable, so equation (IV.10) will be derived from equation (IV.5). Again geometric series will be used, and factors $\left(1-P_{D_{2}}\right), P_{D_{2}},\left(1-P_{D_{3}}\right), P_{D_{3}}$ will be inserted, along with $\rho$ 's and $\rho^{-1}$ 's. Next, we "symmetrize" certain terms, which does not change the sum of all terms. Resumming by geometric series, we obtain equation (IV.10). 


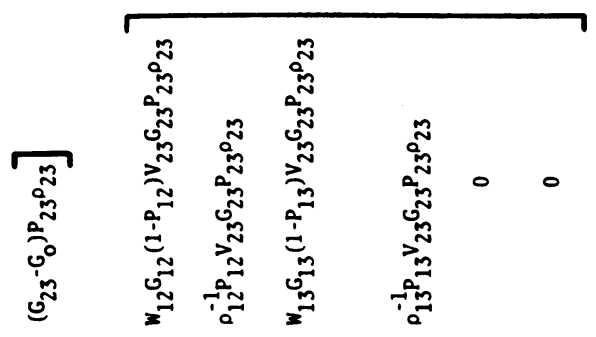

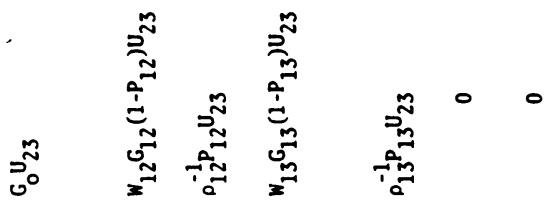
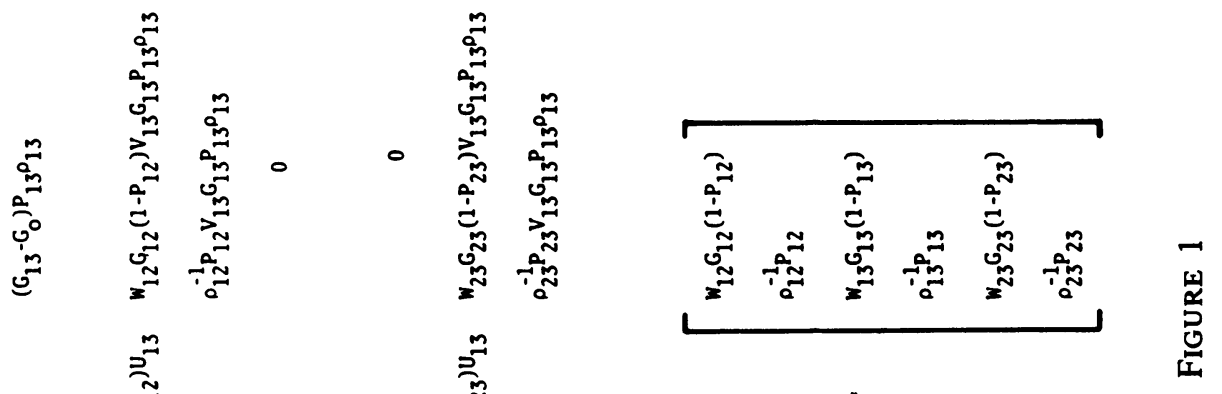

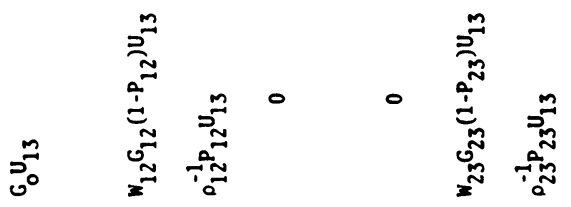

눈
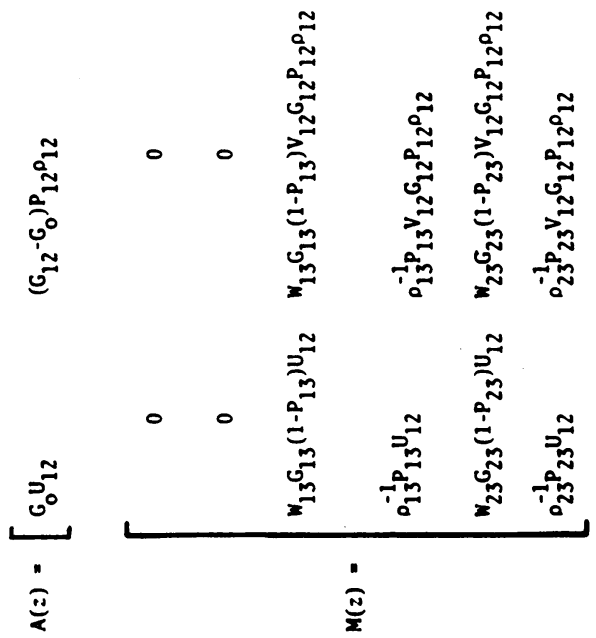
REMARKS. (1) In the three body case, no symmetrization step was necessary because all strings contained only one nontrivial cluster decomposition. In the four body case, some terms require symmetrization. This involves the replacement of certain terms by a combinatorial factor times a sum of those terms.

(2) The symmetrization step allows us to prove asymptotic completeness when the subsystems have embedded (nonpositive) eigenvalues. The equations used by Sigal [26] are not symmetrized, and embedded eigenvalues are not treated by Sigal's asymptotic completeness proof.

(3) Equation (IV.10) is derived from equation (IV.5) by replacing the entries of the $24 \times 24$ matrix of equation (IV.5) by $3 \times 3$ blocks. As a result, equation (IV.10) has an unpleasantly large $72 \times 72$ matrix. An equation equivalent to equation (IV.10) can be derived from equation (IV.4). This formula has a $42 \times 42$ matrix, but the entries are complicated. So, we prefer to use equation (IV.10).

The final resolvent formula for the four body case is

$$
(z-H)^{-1}=\left(z-H_{0}\right)^{-1}+A(z)(1-M(z))^{-1} C(z),
$$

where $A, M$, and $C$ are the $1 \times 72,72 \times 72$, and $72 \times 1$ matrices indicated in the Appendix. A representative sample of the entries is given, and the number associated with each entry refers to the proposition of $\S \mathrm{V}$ in which that entry is studied. $A, M$, and $C$ should be viewed as $1 \times 24,24 \times 24$, and $24 \times 1$ matrices of $1 \times 3,3 \times 3$, and $3 \times 1$ blocks, respectively. The entries are denoted by $A_{S, i}$, $M_{S, i ; S^{\prime}, j}$, and $C_{S, i}$, where $S$ and $S^{\prime}$ denote strings, and $i$ and $j$ run from 1 to 3 .

As in the three body case, equation (IV.10) involves some operators $\rho_{i j}, \rho_{i j, k}$, and $\rho_{i j, k l}$, which will be specified in $\S \mathrm{V}$. Also, equation (IV.10) holds because

$$
A(z)(M(z))^{k} C(z)=\tilde{A}(z)(\tilde{M}(z))^{k} \tilde{C}(z)
$$

for $k>0$. Equation (IV.11) implies equation (IV.10) in the same way equation (IV.7) implied (IV.6). However, the proof of equation (IV.11) is more complicated than that of equation (IV.7).

To prove equation (IV.11), consider first the case $k=0$, and define $Q_{D_{3,1}}=(1-$ $\left.P_{D_{3}}\right), Q_{D_{3}, 2}=P_{D_{3}}, Q_{D_{3}, 3}=1, Q_{D_{2}, 1}=Q_{D_{2}, 2}=\left(1-P_{D_{2}}\right)$, and $Q_{D_{2}, 3}=P_{D_{2}}$. If $S=$ $\left(D_{4}, D_{3}\right)$ is a short string, then

$$
\begin{aligned}
\tilde{A}_{S} \tilde{C}_{S} & =G_{0} V_{D_{3}} G_{D_{3}} \\
& =G_{0} V_{D_{3}} G_{D_{3}} Q_{D_{3}, 1}+G_{0} V_{D_{3}} G_{D_{3}} Q_{D_{3}, 2} \\
& =A_{S, 1} C_{S, 1}+A_{S, 2} C_{S, 2}+0 \\
& =\sum_{i=1}^{3} A_{S, i} C_{S, i} .
\end{aligned}
$$

For long strings $S=\left(D_{4}, D_{3}, D_{2}\right)$, the situation is not so simple because $A_{S, 3}$ has been symmetrized. For each decomposition $D_{2}$ with two clusters, let $\Lambda_{D_{2}}=\left\{S^{\prime}=\right.$ $\left.\left(D_{4}, D_{3}^{\prime}, D_{2}^{\prime}\right): D_{2}^{\prime}=D_{2}\right\}$, and let $l\left(D_{2}\right)$ denote the number of elements of $\Lambda_{D_{2}}$. Then 


$$
\begin{aligned}
& \sum_{S \in \Lambda_{D_{2}}} \tilde{A}_{S} \tilde{C}_{S}=\sum_{D_{3} \text { refines } D_{2}} G_{0} V_{D_{3}} G_{D_{3}}\left(V_{D_{2}}-V_{D_{3}}\right) G_{D_{2}} \\
&=\sum_{D_{3} \text { refines } D_{2}} \sum_{i=1}^{2} G_{0} V_{D_{3}} G_{D_{3}} Q_{D_{3}, i}\left(V_{D_{2}}-V_{D_{3}}\right) G_{D_{2}} Q_{D_{2}, i} \\
&+\sum_{D_{3}^{\prime} \text { refines } D_{2}} G_{0} V_{D_{3}^{\prime}} G_{D_{3}^{\prime}} Q_{D_{3}^{\prime}, 3}\left(V_{D_{2}}-V_{D_{3}^{\prime}}\right) G_{D_{2}} Q_{D_{2}, 3} \\
&= \sum_{S \in \Lambda_{D_{2}}} \sum_{i=1}^{2} A_{S, i} C_{S, i} \\
&+\sum_{D_{3} \text { refines } D_{2}}\left(\frac{1}{l\left(D_{2}\right)} \sum_{D_{3}^{\prime} \text { refines } D_{2}} G_{0} V_{D_{3}^{\prime}} G_{D_{3}^{\prime}} Q_{D_{3_{3}, 3}}\left(V_{D_{2}}-V_{D_{3}^{\prime}}\right) G_{D_{2}} Q_{D_{2}, 3}\right) \\
&= \sum_{S \in \Lambda_{D_{2}}} \sum_{i=1}^{3} A_{S, i} C_{S, i} .
\end{aligned}
$$

Equation (IV.11), for $k=0$, is now obtained from (IV.12) and (IV.13) by the following computation:

$$
\begin{aligned}
\tilde{A} \tilde{C} & =\sum_{S} \tilde{A}_{S} \tilde{C}_{S}=\sum_{S \text { short }} \tilde{A}_{S} \tilde{C}_{S}+\sum_{D_{2}} \sum_{S \in \Lambda_{D_{2}}} \tilde{A}_{S} \tilde{C}_{S} \\
& =\sum_{S \text { short }} \sum_{i=1}^{3} A_{S, i} C_{S, i}+\sum_{D_{2}} \sum_{S \in \Lambda_{D_{2}}} \sum_{i=1}^{3} A_{S, i} C_{S, i} \\
& =\sum_{S} \sum_{i=1}^{3} A_{S, i} C_{S, i}=A C .
\end{aligned}
$$

When $k>0$, the ideas are the same, but more sums are involved. For each sequence $D_{2}^{(1)}, D_{2}^{(2)}, \ldots, D_{2}^{(k)}$, let $\Lambda_{j}=\Lambda_{D_{2}(j)}$. Then, by explicit computation,

$$
\begin{aligned}
\sum_{S_{1} \in \Lambda_{1}} \sum_{S_{2} \in \Lambda_{2}} \cdots & \sum_{S_{k} \in \Lambda_{k}} \tilde{A}_{S_{1}} \tilde{M}_{S_{1}, S_{2}} \tilde{M}_{S_{2}, S_{3}} \ldots \tilde{M}_{S_{k}, S_{k+1}} \tilde{C}_{S_{k+1}} \\
= & \sum_{S_{1} \in \Lambda_{1}} \sum_{S_{2} \in \Lambda_{2}} \ldots \sum_{S_{k} \in \Lambda_{k}} \sum_{i_{1}=1}^{3} \sum_{i_{2}=1}^{3} \ldots \\
& \sum_{i_{k+1}=1}^{3} A_{S_{1}, i_{1}} M_{S_{1}, i_{1} ; S_{2}, i_{2}} \ldots M_{S_{k}, i_{k} ; S_{k+1} i_{k+1}} C_{S_{k+1}, i_{k+1}}
\end{aligned}
$$

for any choice of $S_{k+1}$. This is the generalization of equation (IV.13). Equation (IV.12) does not need to be generalized because $M_{S, S^{\prime}}=0$ whenever $S$ is short. Now equation (IV.11) follows from (IV.14). 


$$
\begin{aligned}
& \tilde{A} \tilde{M}^{k} \tilde{C}=\sum_{S_{1}} \sum_{S_{2}} \ldots \sum_{S_{k}} \sum_{S_{k+1}} \tilde{A}_{S_{1}} \tilde{M}_{S_{1}, S_{2}} \ldots \tilde{M}_{S_{k}, S_{k+1}} \tilde{C}_{S_{k+1}} \\
& =\sum_{S_{1} \text { long }} \sum_{S_{2} \text { long }} \cdots \sum_{S_{k} \text { long }} \sum_{S_{k+1}} \tilde{A}_{S_{1}} \tilde{M}_{S_{1}, S_{2}} \ldots \tilde{M}_{S_{k}, S_{k+1}} \tilde{C}_{S_{k+1}} \\
& =\sum_{D \mathbb{q}^{(1)}} \sum_{D \mathbb{L}^{(2)}} \cdots \sum_{D \mathbb{L}^{(k)}} \sum_{S_{1} \in \Lambda_{1}} \sum_{S_{2} \in \Lambda_{2}} \cdots \\
& \sum_{S_{k} \in \Lambda_{k}} \sum_{S_{k+1}} \tilde{A}_{S_{1}} \tilde{M}_{S_{1}, S_{2}} \ldots \tilde{M}_{S_{k}, S_{k+1}} \tilde{C}_{S_{k+1}} \\
& =\sum_{D 2^{(1)} \ldots D_{2}^{(k)}} \sum_{S_{1} \in \Lambda_{1} \ldots S_{k} \in \Lambda_{k}} \sum_{S_{k+1}} \sum_{i_{1} \ldots i_{k+1}}^{3} A_{S_{1}, i_{1}} M_{S_{1}, i_{1} ; S_{2}, i_{2}} \\
& \ldots M_{S_{k}, i_{k} ; S_{k+1}, i_{k+1}} C_{S_{k+1}, i_{k+1}} \\
& =A M^{k} C \text {. }
\end{aligned}
$$

This establishes equation (IV.11).

In the three body case, equations (IV.8) and (IV.9) show that individual terms in the expression for $A M^{k} C$ correspond to products of blocks of $A, M$, and $C$. This is not true in the four body case, although something closely related is true. Products of certain blocks of $\tilde{A}, \tilde{M}$, and $\tilde{C}$ (determined by the sequence $D_{2}^{(1)}, \ldots, D_{2}^{(k)}$ ) are equal to products of corresponding blocks of $A, M$, and $C$. This is the content of equation (IV.14). Equation (IV.11) is then obtained by summing over the blocks.

Having established equation (IV.11), the validity of equation (IV.10) is proved. In $\S \mathrm{V},(M(z))^{2}$ is shown to be compact, and the bounds required by the multiparticle limiting absorption principle are proved. However, $A(z)$ must first be put into the correct form. This is done by using the following identities:

$$
\text { (1) } G_{0} V_{i j} G_{i j}=G_{i j}-G_{0}
$$

(2) $G_{0}\left(V_{i j} G_{i j}\left(V_{i k}+V_{j k}\right)+V_{i k} G_{i k}\left(V_{i j}+V_{j k}\right)+V_{j k} G_{j k}\left(V_{i j}+V_{i k}\right)\right) G_{i j k}$

$$
\begin{aligned}
= & G_{i j k}-\left(G_{i j} P_{i j}+G_{i k} P_{i k}+G_{j k} P_{j k}\right)-G_{0}\left(1-P_{i j}-P_{i k}-P_{j k}\right) \\
& -G_{0}\left(V_{i j} G_{i j}\left(1-P_{i j}\right)+V_{i k} G_{i k}\left(1-P_{i k}\right)+V_{j k} G_{j k}\left(1-P_{j k}\right)\right),
\end{aligned}
$$

(3) $G_{0}\left(V_{i j} G_{i j} V_{k l}+V_{k l} G_{k l} V_{i j}\right) G_{i j, k l}$

$$
\begin{aligned}
= & G_{i j, k l}-\left(G_{i j} P_{i j}+G_{k l} P_{k l}\right)-G_{0}\left(1-P_{i j}-P_{k l}\right) \\
& -G_{0}\left(V_{i j} G_{i j}\left(1-P_{i j}\right)+V_{k l} G_{k l}\left(1-P_{k l}\right)\right) .
\end{aligned}
$$

Notice that the symmetrization is necessary to put $A$ in a form in which these identities may be used to give a formula of the type required in §III. The other reason for the symmetrization is to make $M(z)$ well behaved as $z$ approaches $\sigma_{\text {ess }}(H)$.

We give in the Appendix a representative sample of the blocks of the matrices $A(z), C(z)$, and $M(z)$. 
V. Estimates. Theorem III.1 and the formulas of $\S I V$ reduce the problem of Kuroda completeness to the proof of certain operator bounds. These bounds are established in this section under the following hypotheses:

(1) $H=H_{0}+\Sigma_{i<j} V_{i j}$ on $L^{2}\left(\mathbf{R}^{(N-1) m}\right)$, where $m \geqslant 3$ and $N \leqslant 4$. Each two body potential $V_{i j}$ is a selfadjoint multiplication operator $U_{i j} W_{i j}$, where $U_{i j}$ and $W_{i j}$ belong to $L_{\delta}^{p}\left(\mathbf{R}^{m}\right)+L_{\delta}^{\infty}\left(\mathbf{R}^{m}\right)$ for some $p>m$ and $\delta>1$.

(2) $U_{i j}: \mathcal{H} \rightarrow \mathcal{H}_{-1}$ and $W_{i j}: \mathcal{H}_{+1} \rightarrow \mathcal{H}$ are dilation analytic.

(3) No cluster Hamiltonian has a bound state or resonance at any of its thresholds.

(4) When $N=4$, we assume the three body cluster Hamiltonians have no positive eigenvalues.

(5) Each cluster Hamiltonian has exactly one bound state.

Remarks. (1) Hypothesis (2) asserts dilation analyticity only. The boundedness of $U_{i j}: \mathcal{H} \rightarrow \mathcal{H}_{-1}$ and $W_{i j}: \mathcal{K}_{+1} \rightarrow \mathcal{H}$ follow from Hypothesis (1) and methods of §II.B.

(2) Bounds established for the cluster Hamiltonians are required to prove bounds for the full Hamiltonian. So, we need two body information to treat the three body case, and two and three body information to treat the four body case.

(3) The threshold set $\Sigma(h)$ for a cluster Hamiltonian $h$ is defined as in §II.E. We prove $(z-h)^{-1}=\left(z-h_{0}\right)^{-1}+A(z)(1-M(z))^{-1} C(z)$, where $M(z)$ has norm continuous boundary values $M(E \pm i 0)$ as $z$ approaches $E \in \mathbf{R}$ from above or below. Hypothesis (3) states that $1 \notin \sigma(M(E \pm i 0))$ whenever $E \in \Sigma(h)$.

(4) By continuity, Hypothesis (3) implies $1 \notin \sigma(M(E \pm i 0))$ for $E$ in the neighborhood of $\Sigma(h)$. We prove $E \notin \Sigma(h)$ and $1 \notin \sigma(M(E \pm i 0))$ imply $E$ is not an eigenvalue of $h$. Thus, $h$ has no eigenvalues near its thresholds. Hypotheses (1) and (2) imply that eigenvalues may accumulate only at thresholds (see Balslev and Combes [3]). So $h$ has finitely many eigenvalues. Therefore, Hypotheses (1)-(3) imply $\boldsymbol{H}$ has finitely many channels.

(5) In §VI, we prove that Hypotheses (1) and (2) imply Hypothesis (3) for a dense open set of couplings.

(6) Hypothesis (5) has been introduced merely for convenience. We denote cluster eigenfunctions $\psi_{12}, \psi_{123}$, etc., and let their corresponding eigenvalues be $E_{12}$, $E_{123}$, etc., respectively. Define $\psi_{12,34}=\psi_{12} \otimes \psi_{34}, \psi_{13,24}=\psi_{13} \otimes \psi_{24}$, and $\psi_{14,23}=$ $\psi_{14} \otimes \psi_{23}$. Similarly, let $E_{12,34}=E_{12}+E_{34}, E_{13,24}=E_{13}+E_{24}$, and $E_{14,23}=E_{14}+$ $E_{23}$.

(7) Theorem II.10 gives sufficient conditions for Hypothesis (4) to hold. Yukawa potentials, generalized Yukawa potentials and potentials of the form $(1+r)^{-2-e}$ are allowed.

Since there are finitely many potentials, Hypothesis (1) implies the existence of a $p>m$ and $\delta>\gamma>1$, such that all $U_{i j}$ and $W_{i j}$ belong to $L_{\delta}^{P}\left(\mathbf{R}^{m}\right)+L_{\delta}^{\infty}\left(\mathbf{R}^{m}\right)$. Fix such $\boldsymbol{a}$ and $p$.

DEFINITION. Let $\xi$ be the coordinate from the center of mass of particles $i$ and $j$ to particle $k$. Let $\zeta$ denote the coordinate from the center of mass of particles $i, j$, and $k$ to particle $l$. Let $\eta$ be the coordinate from the center of mass of particles $i$ and $j$ to the center of mass of particles $k$ and $l$. 

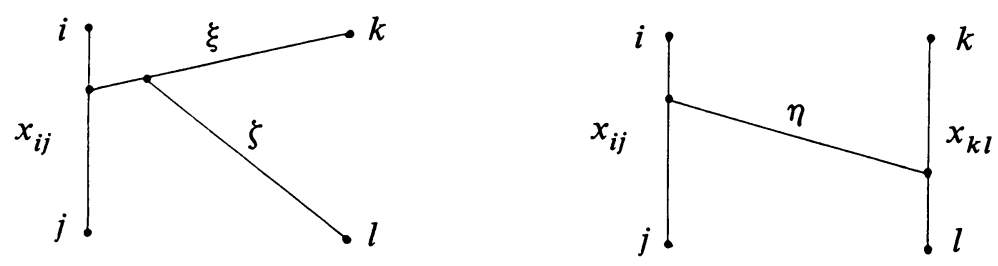

Define

$$
\begin{aligned}
\rho_{i j, k}(\xi) & =\left(1+\xi^{2}\right)^{-\gamma / 2}, \\
\rho_{i j k}(\zeta) & =\left(1+\zeta^{2}\right)^{-\gamma / 2}, \\
\rho_{i j, k l}(\eta) & =\left(1+\eta^{2}\right)^{-\gamma / 2} .
\end{aligned}
$$

In the three body problem, we let $\rho_{i j}=\rho_{i j, k}$ for convenience. In the four body problem we choose functions $\rho_{i j}$ arbitrarily as $\rho_{12}=\rho_{12,3}, \rho_{13}=\rho_{13,2}, \rho_{23}=\rho_{23,1}$, $\rho_{34}=\rho_{34,1}, \rho_{14}=\rho_{14,3}$, and $\rho_{24}=\rho_{24,3}$.

These are the $\rho$ functions introduced in $\S I V$. The choice is certainly not unique, and we have not made the same choice as other authors. However, our $\rho$ functions have the advantage of being dilation analytic.

The results of this section are summarized by the first three propositions. These depend on the technical lemmas which make up most of this section.

Proposition V.1. Assume Hypothesis (1). If $N=2$, then the limiting absorption principle holds for $\boldsymbol{H}$. If Hypothesis (2) also holds, then the exceptional set $\mathcal{E}$ is contained in $(-\infty, 0]$, and $\mathcal{E}$ consists of all eigenvalues of $H$ and possibly the point 0.

Proof. Equation (IV.1) states

$(z-H)^{-1}=\left(z-H_{0}\right)^{-1}+\left(z-H_{0}\right)^{-1} U\left(1-W\left(z-H_{0}\right)^{-1} U\right)^{-1} W\left(z-H_{0}\right)^{-1}$. Let $\rho(x)=\left(1+x^{2}\right)^{-\gamma / 2}$. Define $F_{1}=\rho, F_{2}=U, Z_{1}(z)=\rho^{-1}$, and $Z_{2}(z)=(1-$ $\left.W\left(z-H_{0}\right)^{-1} U\right)^{-1} W\left(z-H_{0}\right)^{-1}$. Then

$$
(z-H)^{-1}=\sum_{i=1}^{2}\left(z-H_{0}\right)^{-1} F_{i} Z_{i}(z) .
$$

Hölder's inequality shows that $F_{1}$ and $F_{2}$ map $L^{2}\left(\mathbf{R}^{m}\right)$ into $L_{\gamma}^{q}\left(\mathbf{R}^{m}\right)+L_{\gamma}^{2}\left(\mathbf{R}^{m}\right)$, where $q^{-1}=p^{-1}+1 / 2$.

Repeating the proof of Lemma II.3, we see that $W\left(z-H_{0}\right)^{-1} U$ is uniformly bounded and analytic for $z \in \mathbf{C} \backslash[0, \infty)$, with norm continuous boundary values as $z$ approaches $[0, \infty)$ from above or below. Similarly, $W\left(z-H_{0}\right)^{-1}$ has these properties as a mapping of $L_{\gamma}^{2}\left(\mathbf{R}^{m}\right)$ into $L^{2}\left(\mathbf{R}^{m}\right)$.

If $U$ and $W$ belong to $L^{2}\left(\mathbf{R}^{m}\right)$, then $W e^{-i t H_{0}} U$ has an $L^{2}$ integral kernel for $t \neq 0$. Thus, $W e^{-i t H_{0}} U$ is compact for $t \neq 0$. When $U$ and $W$ belong to $L_{\gamma}^{p}\left(\mathbf{R}^{m}\right)+$ $L_{\gamma}^{\infty}\left(\mathbf{R}^{m}\right)$, we may approximate them by $L^{2}$ functions, and conclude the compactness 
of $W e^{-i t H_{0}} U$ for $t \neq 0$, by taking limits. Taking Fourier transforms as in Lemma II.3, we see that $W\left(z-H_{0}\right)^{-1} U$ is compact, and $\left\|W\left(z-H_{0}\right)^{-1} U\right\| \rightarrow 0$ as $|\operatorname{Im} z|$ $\rightarrow \infty$ or $\operatorname{Re} z \rightarrow-\infty$.

By the analytic Fredholm theorem and a theorem of Kuroda [16] (see Simon [29]), $\left(1-W\left(z-H_{0}\right)^{-1} U\right)^{-1}$ exists for all $z$ in the closed cut plane, except for some set $\mathcal{E}$. $\mathcal{E} \cap(\mathrm{C} \backslash[0, \infty))$ is discrete; $\mathcal{E} \cap[0, \infty)$ is closed and has Lebesgue measure 0 .

If $w \in \mathcal{E} \cap(\mathrm{C} \backslash[0, \infty))$, then $\phi=W\left(w-H_{0}\right)^{-1} U \phi$ for some $\phi \in \mathcal{H}$. Since $w \notin \sigma\left(H_{0}\right),\left(w-H_{0}\right)^{-1} U$ is bounded, and $\psi=\left(w-H_{0}\right)^{-1} U \phi$ belongs to $\mathcal{H}$. However, $\phi=W\left(w-H_{0}\right)^{-1} U \phi=W \psi$, and $\psi=\left(w-H_{0}\right)^{-1} V \psi$. Applying $(w-$ $H_{0}$ ) to both sides of this equation, we obtain $H \psi=w \psi$. Therefore, $\delta \subseteq \mathbf{R}$ and $\mathcal{E} \cap(-\infty, 0)$ consists of eigenvalues of $H$.

If $w \pm i 0 \in \mathcal{E} \cap[0, \infty)$, and Hypothesis (2) holds, then Lemma II.11 shows that $1 \in \sigma\left(W(\theta)\left(w-H_{0}(\theta)\right)^{-1} U(\theta)\right)$ for some $\theta$ with $\pm \operatorname{Im} \theta>0$. If $w \neq 0$, then $\delta \notin \sigma\left(H_{0}(\theta)\right.$ ), and we can construct $\psi$ (as above), so that $H(\theta) \psi=w \psi$. Theorem II.7 shows that $w$ is an eigenvalue of $H$. Since $w>0$, this contradicts Theorem II.10. Therefore, $\delta \subseteq(-\infty, 0]$ consists of eigenvalues of $H$ and possibly the point 0 .

Proposition V.2 (Ginibre and Moulin [7], Thomas [36], Howland [11]). Assume Hypotheses (1)-(3); if $N=3$, then the multiparticle limiting absorption principle holds for $H$. The exceptional set $\mathcal{E}$ is at most countable, and consists of eigenvalues of $\boldsymbol{H}$ and possibly some thresholds of $H$.

Proof. For convenience, we also assume Hypothesis (5).

As in the proof of Proposition V.1, we begin by writing

$$
(z-H)^{-1}=\sum_{D}\left(z-H_{D}\right)^{-1} P_{D} \sum_{l} F_{l, D} Z_{l, D}(z)
$$

This expression is obtained from equation (IV.6). The $F_{l, D}$ 's are $U_{i j}$ 's, $\rho_{i j}$ 's, and $P_{i j}$ $\rho_{i j}$ 's. The $Z_{l, D}(z)$ 's are made up of components of $(1-M(z))^{-1} C(z)$.

$C(z): L_{\gamma}^{2}\left(\mathbf{R}^{2 m}\right) \rightarrow \bigoplus_{i=1}^{6} L^{2}\left(\mathbf{R}^{2 m}\right)$ is analytic and uniformly bounded for $z \in \mathbf{C} \backslash$ $\sigma_{\text {ess }}(H)$, with norm continuous boundary values as $z$ approaches $\sigma_{\text {ess }}(H)$ from above or below. This is proved by Lemmas V.4 and V.8 below.

$M(z): \bigoplus_{i=1}^{6} L^{2}\left(\mathbf{R}^{2 m}\right) \rightarrow \bigoplus_{i=1}^{6} L^{2}\left(\mathbf{R}^{2 m}\right)$ has all the properties mentioned above for $C(z)$. In addition, $(M(z))^{2}$ is compact and $\left\|(M(z))^{2}\right\| \rightarrow 0$ as $\operatorname{Re} z \rightarrow-\infty$. These facts are proved by Lemma V.13.

Let $\mathcal{E}$ be the set of $z$, such that $(1-M(z))^{-1}$ does not exist. If $z \in \mathcal{E} \cap(\mathrm{C} \backslash$ $\left.\sigma_{\text {ess }}(H)\right)$, then the compactness of $(M(z))^{2}$ shows that $M(z) \phi=\phi$ for some $\phi \in$ $\bigoplus_{i=1}^{6} \mathcal{H}$;

$$
\phi_{\alpha, i}=\sum_{\beta, j} M_{\alpha, i ; \beta, j}(z) \phi_{\beta,}
$$


Define $\eta_{\alpha}=\phi_{\alpha, 1}+W_{\alpha}\left(z-H_{\alpha}\right)^{-1} P_{\alpha} \rho_{\alpha} \phi_{\alpha, 2}$. Then by the relation of $M(z)$ and T( $(z)$,

$$
\begin{aligned}
\eta_{\alpha} & =\sum_{\beta} \Re_{\alpha, \beta}(z) \eta_{\beta}=\sum_{\beta \neq \alpha} W_{\alpha}\left(z-H_{\alpha}\right)^{-1} U_{\beta} \eta_{\beta} \\
& =\sum_{\beta \neq \alpha} W_{\alpha}\left(z-H_{0}\right)^{-1} U_{\beta} \eta_{\beta}+\sum_{\beta \neq \alpha} W_{\alpha}\left(z-H_{0}\right)^{-1} V_{\alpha}\left(z-H_{\alpha}\right)^{-1} U_{\beta} \eta_{\beta} \\
& =\sum_{\beta \neq \alpha} W_{\alpha}\left(z-H_{0}\right)^{-1} U_{\beta} \eta_{\beta}+W_{\alpha}\left(z-H_{0}\right)^{-1} U_{\alpha} \eta_{\alpha} \\
& =\sum_{\beta} W_{\alpha}\left(z-H_{0}\right)^{-1} U_{\beta} \eta_{\beta} .
\end{aligned}
$$

Since $z \notin \sigma_{\text {ess }}(H),\left(z-H_{0}\right)^{-1} U_{\beta}$ is bounded. Hence, $\psi=\left(z-H_{0}\right)^{-1} \Sigma_{\beta} U_{\beta} \eta_{\beta}$ belongs to $\mathcal{H}$. Moreover, equation (V.1) shows $\eta_{\alpha}=W_{\alpha} \psi$, so $\psi=\left(z-H_{0}\right)^{-1}\left(V_{12}\right.$ $\left.+V_{13}+V_{23}\right) \psi$. Therefore, $H \psi=z \psi$.

If $z \pm i 0 \in \mathcal{E} \cap \sigma_{\text {ess }}(H)$, and $z$ is not a threshold, then Lemma II.11 shows that $z \in \mathcal{E}$ implies $1 \in \sigma(M(z, \theta))$, for some $\theta$ with $\pm \operatorname{Im} \theta>0$. Since $z \notin \sigma \sigma_{\text {ess }}(H(\theta))$, equation (V.1) shows $H(\theta) \psi=z \psi$ for some $\psi \in \mathcal{H}$. Theorem II.7 shows that $z$ is an eigenvalue of $\boldsymbol{H}$.

Since $M(z)$ depends continuously on $z, \mathcal{E}$ is a closed set. Since eigenvalues of $H$ may accumulate only at thresholds, $\mathcal{E}$ is at most countable, and has Lebesgue measure zero.

Proposition V.3. Assume Hypotheses (1)-(4). If $N=4$, then the multiparticle limiting absorption principle holds for $\boldsymbol{H}$.

Proof. Using equation (IV.10) and the identities (IV.15), we may write ( $z-$ $H)^{-1}$ in the required form.

$C(z): L_{\gamma}^{2}\left(\mathbf{R}^{3 m}\right) \rightarrow \bigoplus_{i=1}^{72} L^{2}\left(\mathbf{R}^{3 m}\right)$ is analytic and uniformly bounded for $z \in \mathbf{C} \backslash$ $\sigma_{\text {ess }}(H)$, with norm continuous boundary values as $z$ approaches $\sigma_{\text {ess }}(H)$ from above or below. This is proved by Corollary V.5, Lemma V.8, and Propositions V.15, V.16, V.25, and V.26.

$M(z): \bigoplus_{i=1}^{72} L^{2}\left(\mathbf{R}^{3 m}\right) \rightarrow \bigoplus_{i=1}^{72} L^{2}\left(\mathbf{R}^{3 m}\right)$ is analytic and uniformly bounded for $z \in \mathbf{C} \backslash \sigma_{\text {ess }}(H)$, with norm continuous boundary values as $z$ approaches $\sigma_{\text {ess }}(H)$ from above or below. $(M(z))^{2}$ is compact, and $\left\|(M(z))^{2}\right\| \rightarrow 0$ as $\operatorname{Re} z \rightarrow-\infty$. The compactness of $M^{2}$ follows from the fact that only the lower left corner entries in any of the $3 \times 3$ blocks for $M$ may fail to be compact. Also, these are the only ones which do not approach zero as $\operatorname{Re} z \rightarrow-\infty$.

These facts about $M(z)$ are proved by Lemma V.4 and Propositions V.15-V.20, V.25-V.32.

The exceptional set $\mathcal{E}$ is defined as the set of all real $z$ such that $1 \in \sigma(M(z))$. The analytic Fredholm theorem and a theorem of Kuroda [16] (see also Simon [29]) show that $\mathcal{E}$ is a closed set of measure zero.

The only facts remaining to be proved are the boundedness, analyticity, and continuity up to $\sigma_{\text {ess }}(H)$ of certain factors which occur in $A(z)$. These facts are proved by Lemma V.8. 
Lemma V.4. Assume Hypotheses (1)-(3), and (5). Let $i, j, k$, and $l$ be distinct indices. Choose coordinates $\xi, \zeta$, and $\eta$ as in the definitions of $\rho_{i j, k}, \rho_{i j k}$, and $\rho_{i j, k l}$. Then, for all $\alpha, \beta, \delta \in \mathbf{R}$,

(a) $\rho_{i j, k}^{-1} P_{i j} U_{i k}$ is bounded from $L_{-\beta}^{2}\left(\mathbf{R}^{m}, d x_{i j}\right) \otimes L_{\delta}^{2}\left(\mathbf{R}^{2 m}, d \xi d \zeta\right)$ to $L_{\alpha}^{2}\left(\mathbf{R}^{m}, d x_{i j}\right) \otimes$ $L_{\delta}^{2}\left(\mathbf{R}^{2 m}, d \xi d \zeta\right)$.

(b) $\rho_{i j k}^{-1} P_{i j k} U_{k l}$ is bounded from $L_{-\beta}^{2}\left(\mathbf{R}^{2 m}, d x_{i j} d \xi\right) \otimes L_{\delta}^{2}\left(\mathbf{R}^{m}, d \zeta\right)$ to $L_{\alpha}^{2}\left(\mathbf{R}^{2 m}, d x_{i j} d \xi\right)$ $\otimes L_{\delta}^{2}\left(\mathbf{R}^{m}, d \zeta\right)$.

(c) $\rho_{i j, k l}^{-1} P_{i j, k l} U_{i k}$ is bounded from $L_{-\beta}^{2}\left(\mathbf{R}^{2 m}, d x_{i j} d x_{k l}\right) \otimes L_{\delta}^{2}\left(\mathbf{R}^{m}, d \eta\right)$ to $L_{\alpha}^{2}\left(\mathbf{R}^{2 m}, d x_{i j} d x_{k l}\right) \otimes L_{\delta}^{2}\left(\mathbf{R}^{m}, d \eta\right)$.

Proof. First, we prove three inequalities involving coordinates:

$$
\begin{aligned}
2\left(1+x_{i j}^{2}\right)\left(1+x_{i k}^{2}\right)-\left(1-\xi^{2}\right) & \\
= & 1+2 x_{i j}^{2} x_{i k}^{2}+x_{i k}^{2}-2 m_{j}\left(m_{i}+m_{j}\right)^{-1} x_{i j} \cdot x_{i k} \\
& +\left(2-m_{j}^{2}\left(m_{i}+m_{j}\right)^{-2}\right) x_{i j}^{2} \\
\geqslant & 1+2 x_{i j}^{2} x_{i k}^{2}+\left(x_{i k}-m_{j}\left(m_{i}+m_{j}\right)^{-1} x_{i j}\right)^{2} \geqslant 0,
\end{aligned}
$$

where we have used $\left(2-m_{j}^{2}\left(m_{i}+m_{j}\right)^{-2}\right) \geqslant 1>m_{j}^{2}\left(m_{i}+m_{j}\right)^{-2}$. Therefore,

$$
\left(1+\xi^{2}\right) \leqslant 2\left(1+x_{i j}^{2}\right)\left(1+x_{i k}^{2}\right) \text {. }
$$

The same computation (but with different masses) shows

$$
\left(1+\zeta^{2}\right) \leqslant 2\left(1+x_{k l}^{2}\right)\left(1+\xi^{2}\right)
$$

Once again, the same computation (with different masses again) shows: $\left(1+\eta^{2}\right)$ $<2\left(1+\xi^{2}\right)\left(1+x_{k l}^{2}\right)$. Combining this with inequality (V.2), we obtain

$$
\left(1+\eta^{2}\right)<4\left(1+x_{i j}^{2}\right)\left(1+x_{i k}^{2}\right)\left(1+x_{k l}^{2}\right) .
$$

Since Hypotheses (1)-(3) are satisfied, Theorem II.9 shows that $\psi_{i j}\left(x_{i j}\right), \psi_{i j k}\left(x_{i j}, \xi\right)$, and $\psi_{i j, k l}\left(x_{i j}, x_{k l}\right)$ fall off exponentially. It is therefore sufficient to consider only the case $\alpha=\beta=0$. Also, it suffices to consider only $\delta=0$, because $\rho_{i j, k}^{-1} P_{i j} U_{i k}$ commutes with multiplication by functions of $\xi$ and $\zeta, \rho_{i j k}^{-1} P_{i j k} U_{k l}$ commutes with multiplication by functions of $\zeta$, and $\rho_{i j, k l}^{-1} P_{i j, k l} U_{i k}$ commutes with multiplication by functions of $\eta$.

$$
\begin{aligned}
\rho_{i j, k}^{-1} P_{i j} U_{i k}= & P_{i j} U_{i k} \rho_{i j, k}^{-1} \\
= & {\left[P_{i j}\left(1+x_{i j}^{2}\right)^{\delta / 2}\left(1-\Delta_{i j}\right)^{1 / 2}\right]\left[\left(1-\Delta_{i j}\right)^{-1 / 2} U_{i k}\left(1+x_{i k}^{2}\right)^{\delta / 2}\right] } \\
& \times\left[\left(1+x_{i k}^{2}\right)^{-\delta / 2}\left(1+x_{i j}^{2}\right)^{-\delta / 2} \rho_{i j, k}^{-1}\right] .
\end{aligned}
$$

The first factor is bounded because $\left(1+x_{i j}^{2}\right)^{\delta / 2} \psi_{i j}$ belongs to the quadratic form domain of $-\Delta_{i j}$ (Theorem II.9). The third factor is bounded because of inequality (V.2).

The second factor is bounded if its absolute square, $\left(1+x_{i k}^{2}\right)^{\delta / 2} \bar{U}_{i k}(1-$ $\left.\Delta_{i j}\right)^{-1} U_{i k}\left(1+x_{i k}^{2}\right)^{\delta / 2}$, is bounded. To bound the absolute square, we write it as a 
Laplace transform:

$$
\int_{0}^{\infty} \overline{F\left(x_{i k}\right)} e^{t \Delta_{i j}} F\left(x_{i k}\right) e^{-t} d t
$$

where $F\left(x_{i k}\right)=\left(1+x_{i k}^{2}\right)^{\delta / 2} U_{i k}\left(x_{i k}\right)$.

Suppose $\phi \in L^{2}\left(\mathbf{R}^{3 m}\right)$. To compute $\left\|F\left(x_{i k}\right) e^{t \Delta_{i j}} F\left(x_{i k}\right) \phi\right\|$, we use coordinates $x_{i j}, \xi$, $\zeta$, and first compute the $L^{2}$-norm in the $x_{i j}$ variable with $\xi$ and $\zeta$ fixed. The operator $e^{t \Delta_{v}}$ has an explicitly known integral kernel [22] and $x_{i j}=c_{1} x_{i j}+c_{2} \xi$, with $c_{1} \neq 0$, since all masses are finite: $F\left(x_{i k}\right) \in L^{P}\left(\mathbf{R}^{m}, d x_{i k}\right)+L^{\infty}\left(\mathbf{R}^{m}, d x_{i k}\right)$, for some $p>m$. So, for each fixed $\xi$ and $\zeta, F\left(c_{1} x_{i j}+c_{2} \xi\right) \phi\left(x_{i j}, \xi, \zeta\right)$ belongs to $L^{r}\left(\mathbf{R}^{m}, d x_{i j}\right)+$ $L^{2}\left(\mathbf{R}^{m}, d x_{i j}\right)$, where $r^{-1}=p^{-1}+1 / 2$. If $t>0, e^{t \Delta_{i j}}$ maps this into $L^{2}\left(\mathbf{R}^{m}, d x_{i j}\right) \cap$ $L^{\infty}\left(\mathbf{R}^{m}, d x_{i j}\right)$ (where we have used the fact that $r<2$ ). Multiplication by $\overline{F\left(c_{1} x_{i j}+c_{2} \xi\right)}$ maps this into $L^{2}\left(\mathbf{R}^{m}, d x_{i j}\right)$. By keeping track of the norms at each stage, we obtain:

$$
\begin{gathered}
\int\left|e^{-t}\left(F\left(x_{i k}\right) e^{t \Delta_{i j}} F\left(x_{i k}\right) \phi\right)\left(x_{i j}, \xi, \zeta\right)\right|^{2} d x_{i j} \\
\leqslant C_{F} f(t) \int\left|\phi\left(x_{i j}, \xi, \zeta\right)\right|^{2} d x_{i j}
\end{gathered}
$$

where $C_{F}$ is a constant depending only on $F$ and $\int_{0}^{\infty}(f(t))^{1 / 2} d t$ is finite.

Integrating over $\xi$ and $\zeta$, we see that

$$
\left\|\overline{F\left(x_{i k}\right)} e^{t \Delta i j} F\left(x_{i k}\right) e^{-t} \phi\right\| \leqslant C_{F}(f(t))^{1 / 2}\|\phi\|,
$$

which belongs to $L^{1}((0, \infty), d t)$. The Laplace transform now yields part (a) of the lemma.

Parts (b) and (c) are proved by using the same methods, with inequalities (V.3) and (V.4) in place of (V.2).

COROllary V.5. Let $i, j, k$, and $l$ be distinct indices. Let $\delta \in \mathbf{R}$.

(a) $\rho_{i j, k}^{-1}, \rho_{i j k}^{-1}$, and $\rho_{i j, k l}^{-1}$ are bounded from $L_{\delta+\gamma}^{2}\left(\mathbf{R}^{3 m}\right)$ to $L_{\delta}^{2}\left(\mathbf{R}^{3 m}\right)$.

(b) Assume Hypotheses (1)-(3), and (5), and let $n$ equal $j, k$, or $l$.

For any $\alpha$ and $\beta$, the following operators are bounded:

$$
\begin{gathered}
W_{i n} P_{i j}: L_{-\beta}^{2}\left(\mathbf{R}^{m}, d x_{i j}\right) \otimes L_{\delta}^{2}\left(\mathbf{R}^{2 m}, d \xi d \zeta\right) \rightarrow L_{\alpha}^{2}\left(\mathbf{R}^{m}\right) \otimes L_{\delta}^{2}\left(\mathbf{R}^{2 m}, d \xi d \zeta\right), \\
W_{i n} P_{i j k}: L_{-\beta}^{2}\left(\mathbf{R}^{2 m}, d x_{i j} d \xi\right) \otimes L_{\delta}^{2}\left(\mathbf{R}^{m}, d \zeta\right) \rightarrow L_{\alpha}^{2}\left(\mathbf{R}^{2 m}, d x_{i j} d \xi\right) \otimes L_{\delta}^{2}\left(\mathbf{R}^{m}, d \zeta\right), \\
W_{i n} P_{i j, k l}: L_{-\beta}^{2}\left(\mathbf{R}^{2 m}, d x_{i j} d x_{k l}\right) \otimes L_{\delta}^{2}\left(\mathbf{R}^{m}, d \eta\right) \rightarrow L_{\alpha}^{2}\left(\mathbf{R}^{2 m}, d x_{i j} d x_{k l}\right) \otimes L_{\delta}^{2}\left(\mathbf{R}^{m}, d \eta\right) .
\end{gathered}
$$

Proof. Part (a) follows immediately from the definitions of the $\rho$ 's and $L_{\delta^{-}}^{2}$ spaces.

If $n \neq j$, then Lemma V.4 yields the boundedness of $W_{i n} P_{i j}=W_{i n} P_{i j} \rho_{i j, n}^{-1} \rho_{i j, n}$ on the weighted spaces, because $\rho_{i j, n}$ is bounded. If $n=j$, we use the fact that the operator domain of $W_{i j}$ contains the operator domain of $H_{0}^{1 / 2}$ (see §II.B). Theorem II.9 shows that $e^{a\left|x_{i j}\right|} \psi_{i j}$ belongs to the domain of $H_{0}^{1 / 2}$, so $W_{i j} P_{i j}$ is bounded on the given weighted spaces.

The same methods apply to the remaining operators of part (b). 
Definition. Suppose $A(z): L_{\alpha}^{2}\left(\mathbf{R}^{n}\right) \rightarrow L_{\beta}^{2}\left(\mathbf{R}^{n}\right)$ is an operator valued function, which has the following properties:

(i) $A(z)$ is analytic for $z \in \mathrm{C} \backslash \sigma_{\text {ess }}(H)$.

(ii) $A(z)$ is uniformly bounded for $\operatorname{Re} z \leqslant C$.

(iii) $\|A(z)\| \rightarrow 0$ as $\operatorname{Re} \rightarrow-\infty$.

(iv) $A(z)$ has norm continuous extensions $A(x \pm i 0)$ to $\sigma_{\text {ess }}(H)$ from above and below $\left(A\left(x_{0}+i 0\right)=A\left(x_{0}-i 0\right)\right.$ if $x_{0}=$ inf $\left.\sigma_{\text {ess }}(H)\right)$. Then $A(z)$ is called well behaved from $L_{\alpha}^{2}\left(\mathbf{R}^{n}\right)$ to $L_{\beta}^{2}\left(\mathbf{R}^{n}\right)$. If $\alpha=\beta=0$, then we say $A(z)$ is well behaved.

Lemma V.6. Assume Hypotheses (1)-(3), and (5). Let $i, j, k$ and $l$ be distinct indices. Let $D_{1}$ and $D_{2}$ be cluster decompositions. The following are well behaved:

$$
\begin{aligned}
& K_{1}(z)=W_{i j}\left(z-H_{0}\right)^{-1} U_{i k}, \quad K_{2}(z)=W_{i j}\left(z-H_{k l}\right)^{-1} U_{i k}, \\
& K_{3}(z)=W_{i j} P_{D_{1}}\left(z-H_{0}\right)^{-1} U_{i k}, \quad K_{4}(z)=W_{i j} P_{D_{1}}\left(z-H_{k l}\right)^{-1} U_{i k}, \\
& K_{5}(z)=W_{i j}\left(z-H_{0}\right)^{-1} P_{D_{2}} U_{i k}, \quad K_{6}(z)=W_{i j}\left(z-H_{k l}\right)^{-1} P_{D_{2}} U_{i k}, \\
& K_{7}(z)=W_{i j} P_{D_{1}}\left(z-H_{0}\right)^{-1} P_{D_{2}} U_{i k}, \text { and } K_{8}(z)=W_{i j} P_{D_{1}}\left(z-H_{k l}\right)^{-1} P_{D_{2}} U_{i k} .
\end{aligned}
$$

Proof. Lemma II.3 yields the result for $K_{1}$ and $K_{2}$. If $P_{D_{1}}$ commutes with $W_{i j}$, then Lemma II.3 yields the result for $K_{3}$ and $K_{4}$, also. If $i$ and $j$ belong to the same cluster of $D_{1}$, then Corollary V.5 shows that $W_{i j} P_{D_{1}}\left(1+x_{i j}^{2}\right)$ is bounded. However, Lemma II.3 applies to $\left(1+x_{i j}^{2}\right)^{-1}\left(z-H_{0}\right)^{-1} U_{i k}$ and $\left(1+x_{i j}^{2}\right)^{-1}\left(z-H_{k l}\right)^{-1} U_{i k}$. So, it suffices to consider $K_{3}$ and $K_{4}$ when $i$ and $j$ belong to different clusters of $D_{1}$, but $W_{i j}$ and $P_{D_{1}}$ do not commute. In that case, Lemma V.4 shows that the adjoint of $W_{i j} P_{D_{1}} \rho^{-1}$ is bounded into some tensor product of weighted spaces. Thus, $W_{i j} P_{D_{1}} \rho^{-1}$ is bounded. Lemma II.3 shows that $\rho P_{D_{1}}\left(z-H_{0}\right)^{-1} U_{i k}$ and $\rho P_{D_{1}}(z-$ $\left.H_{k l}\right)^{-1} U_{i k}$ are well behaved. So, $K_{3}$ and $K_{4}$ are well behaved. Of course, the correct choice of $\rho$ must be made.

The same methods control $K_{5}, K_{6}, K_{7}$, and $K_{8}$.

Lemma V.7. Assume Hypotheses (1)-(3). Suppose $N \geqslant 3$, and $\alpha$ is any pair. Then $K(z)=W_{\alpha}\left(1-P_{\alpha}\right)\left(z-H_{\alpha}\right)^{-1} U_{\alpha}$ is well behaved.

Proof. We may decompose $\mathcal{H}$ as a direct integral of Hilbert spaces for the two body problem determined by the pair $\alpha . K(z)$ is fibered under this decomposition, and it suffices to control the operators on the individual fibers. So, we need only show $W_{\alpha}\left(1-P_{\alpha}\right)\left(z-h_{\alpha}\right)^{-1} U_{\alpha}$ is well behaved on $L^{2}\left(\mathbf{R}^{m}\right)$ :

$$
\begin{aligned}
& W_{\alpha}\left(1-P_{\alpha}\right)\left(z-h_{\alpha}\right)^{-1} U_{\alpha}=W_{\alpha}\left(1-P_{\alpha}\right)\left(z-h_{0}\right)^{-1} U_{\alpha} \\
& \quad+W_{\alpha}\left(1-P_{\alpha}\right)\left(z-h_{0}\right)^{-1} U_{\alpha}\left(1-W_{\alpha}\left(z-h_{0}\right)^{-1} U_{\alpha}\right)^{-1} W_{\alpha}\left(z-h_{0}\right)^{-1} U_{\alpha} .
\end{aligned}
$$

In view of Lemma V.6, it suffices to show that the factor of $\left(1-P_{\alpha}\right)$ cancels the singularities of $\left(1-W_{\alpha}\left(z-h_{0}\right)^{-1} U_{\alpha}\right)^{-1}$.

Let $M(z)=W_{\alpha}\left(z-h_{0}\right)^{-1} U_{\alpha}$. The proof of Proposition V.1 shows that $(1-$ $M(z))^{-1}$ is singular when $z$ is a real negative eigenvalue of $h_{\alpha}$, and possibly when $z=0$. Hypothesis (3) implies that it is not singular at $z=0$. 
The poles of $(1-M(z))^{-1}$ are first order poles. To prove this, let $z_{0}$ be an eigenvalue of $h_{\alpha}$, and consider the Laurent series

$$
(1-M(z))^{-1}=\sum_{k=-n}^{\infty}\left(z-z_{0}\right)^{k} L_{k},
$$

where $L_{-n} \neq 0$. Since $L_{-n} \neq 0$, there is a vector $\phi$ in the domain of $U_{\alpha}$, such that $L_{-n} \phi \neq 0$. $\left[(1-M(z))^{-1}-1\right] \phi=(1-M(z))^{-1} M(z) \phi$ must have a pole of order $n$, so $\psi=M\left(z_{0}\right) \phi=C\left(z_{0}\right)\left(U_{\alpha} \phi\right)$ is a nonzero element of $\operatorname{Ran} C(z)$ with $L_{-n} \psi \neq 0$. Similarly, using

$$
\left[(1-M(z))^{-1}-1\right]=M(z)(1-M(z))^{-1}
$$

we see that every nonzero $\eta \in \operatorname{Ran} L_{n}$ has $A\left(z_{0}\right) \eta \neq 0$. Therefore,

$$
\left(z-h_{\alpha}\right)^{-1}\left(U_{\alpha} \phi\right)=\left(z-h_{0}\right)^{-1}\left(U_{\alpha} \phi\right)+A(z)(1-M(z))^{-1} C(z)\left(U_{\phi}\right)
$$

has a pole of order $n$. Since $h_{\alpha}$ is selfadjoint, $n=1$.

If $Q=L_{-1}$ is the residue at $z_{0}$, then $Q$ is a projection onto solutions of $M\left(z_{0}\right) \phi=\phi$ (see Kato [14]). If $P$ is the orthogonal projection onto all eigenvectors of $h_{\alpha}$ with eigenvalue $z_{0}$, then $P A\left(z_{0}\right)=A\left(z_{0}\right) Q$. (This is proved in the proof of Proposition V.1.) Therefore, for $z$ near $z_{0}$,

$$
\begin{aligned}
&(1-P) A(z)(1-M(z))^{-1} \\
&=(1-P) A\left(z_{0}\right)(1-M(z))^{-1}-\left(z-z_{0}\right)(1-P) \\
& \times\left(z-h_{0}\right)^{-1} A\left(z_{0}\right)(1-M(z))^{-1} \\
&= A\left(z_{0}\right)(1-Q) \sum_{k=-1}^{\infty}\left(z-z_{0}\right)^{k} L_{k}-(1-P) \\
& \times\left(z-h_{0}\right)^{-1} A\left(z_{0}\right) \sum_{k=-1}^{\infty}\left(z-z_{0}\right)^{k+1} L_{k} \\
&= A\left(z_{0}\right)(1-Q) \sum_{k=0}^{\infty}\left(z-z_{0}\right)^{k} L_{k}-(1-P) \\
& \times\left(z-h_{0}\right)^{-1} A\left(z_{0}\right) \sum_{k=-1}^{\infty}\left(z-z_{0}\right)^{k+1} L_{k} .
\end{aligned}
$$

Thus, $(1-P) A(z)(1-M(z))^{-1}$ has a removable singularity at $z_{0}$. Since $\left(1-P_{\alpha}\right)$ $=\left(1-P_{\alpha}\right)(1-P)$, the factor $\left(1-P_{\alpha}\right)$ cancels the singularities of $(1-M(z))^{-1}$ and the lemma is proved.

LEMMA V.8. Assume Hypotheses (1)-(3), and (5). Let $\alpha, \beta$, and $\delta$ be pairs such that $\alpha$ and $\delta$ have at least one index in common. Then $K_{1}(z)=W_{\alpha}\left(1-P_{\beta}\right)\left(z-H_{\beta}\right)^{-1} U_{\delta}$ is well behaved, and $K_{2}(z)=W_{\alpha}\left(1-P_{\beta}\right)\left(z-H_{\beta}\right)^{-1}$ is well behaved from $L_{\gamma}^{2}\left(\mathbf{R}^{(N-1) m}\right)$ to $L^{2}\left(\mathbf{R}^{(N-1) m}\right)$. 
Proof. Consider only $K_{1}$; proof for $K_{2}$ is similar.

If $\beta$ has no index in common with $\alpha$, or if $\beta$ has no index in common with $\delta$, then Lemma V.6 applies. Thus, we may assume $\beta$ has indices in common with $\alpha$ and $\delta$ :

$$
\begin{aligned}
& W_{\alpha}\left(1-P_{\beta}\right)\left(z-H_{\beta}\right)^{-1} U_{\delta} \\
&= W_{\alpha}\left(1-P_{\beta}\right)\left(z-H_{0}\right)^{-1} U_{\delta}+W_{\alpha}\left(1-P_{\beta}\right)\left(z-H_{\beta}\right)^{-1} V_{\beta}\left(z-H_{0}\right)^{-1} U_{\delta} \\
&= W_{\alpha}\left(1-P_{\beta}\right)\left(z-H_{0}\right)^{-1} U_{\delta}+W_{\alpha}\left(z-H_{\beta}\right)^{-1}\left(1-P_{\beta}\right) V_{\beta}\left(z-H_{0}\right)^{-1} U_{\delta} \\
&= W_{\alpha}\left(1-P_{\beta}\right)\left(z-H_{0}\right)^{-1} U_{\delta} \\
&+\left(W_{\alpha}\left(z-H_{0}\right)^{-1}\left(1-P_{\beta}\right) U_{\beta}\right)\left(W_{\beta}\left(z-H_{0}\right)^{-1} U_{\delta}\right) \\
&+\left(W_{\alpha}\left(z-H_{0}\right)^{-1} U_{\beta}\right)\left(W_{\beta}\left(1-P_{\beta}\right)\left(z-H_{\beta}\right)^{-1} U_{\beta}\right)\left(W_{\beta}\left(z-H_{0}\right)^{-1} U_{\delta}\right) .
\end{aligned}
$$

Apply Lemmas V.6 and V.7.

Lemma V.9. Assume Hypotheses (1)-(3), and (5). Let $\alpha, \beta$, $\delta$, and $\sigma$ be pairs with $\alpha \neq \beta$ and $\beta \neq \sigma$. If $\alpha$ and $\beta$ have no index in common, assume $\beta=\delta$ or that $\delta$ and $\sigma$ have an index in common. Let $D$ be any cluster decomposition, such that the indices of $\alpha$ belong to the same cluster and the indices of $\beta$ belong to the same cluster. The following are well behaved:

$$
\begin{aligned}
& K_{1}(z)=W_{\alpha}\left(z-H_{0}\right)^{-1} V_{\beta}\left(z-H_{0}\right)^{-1} U_{\sigma}, \\
& K_{2}(z)=W_{\alpha}\left(1-P_{\alpha}\right)\left(z-H_{\alpha}\right)^{-1} V_{\beta}\left(z-H_{0}\right)^{-1} U_{\sigma}, \\
& K_{3}(z)=W_{\alpha}\left(1-P_{\alpha}\right)\left(z-H_{\alpha}\right)^{-1} V_{\beta}\left(1-P_{\delta}\right)\left(z-H_{\delta}\right)^{-1} U_{\sigma}, \\
& K_{4}(z)=W_{\alpha}\left(1-P_{\alpha}\right)\left(z-H_{\alpha}\right)^{-1} V_{\beta} P_{D}\left(z-H_{0}\right)^{-1} U_{\sigma}, \\
& K_{5}(z)=W_{\alpha}\left(1-P_{\alpha}\right)\left(z-H_{\alpha}\right)^{-1} V_{\beta} P_{D}\left(1-P_{\delta}\right)\left(z-H_{\delta}\right)^{-1} U_{\sigma} .
\end{aligned}
$$

Proof (SEe Iorio and O'CARrol [13]). Lemma II.3 shows that $K_{1}$ is well behaved if it has norm continuous boundary values. If $\alpha$ and $\beta$ have an index in common and $\beta$ and $\sigma$ have an index in common, then Lemma II.3 shows $K_{1}$ is well behaved.

If $\alpha$ and $\beta$, or $\beta$ and $\sigma$ have no index in common, then, by taking adjoints if necessary, we may assume $\beta$ and $\sigma$ have no index in common. Then, for $\operatorname{Im} z>0$, $\operatorname{Im} w>0$,

$$
\begin{aligned}
\left|\left\langle\phi\left(K_{1}(z)-K_{1}(w)\right) \psi\right\rangle\right| & \\
= & \left|\int_{0}^{\infty} \int_{0}^{\infty}\left\langle\phi, W_{\alpha} e^{-i t H_{0}} V_{\beta} e^{-i s H_{0}} U_{\sigma} \psi\right\rangle\left(e^{i(t+s) z}-e^{i(t+s) w}\right) d s d t\right| \\
< & \int_{0}^{\infty} \int_{0}^{\infty}\left|\left\langle\phi, W_{\alpha} e^{-i t H_{0}} e^{-i s\left(H_{0}-H_{\beta}^{\beta}\right)} U_{\sigma} V_{\beta} e^{-i s H \beta} \psi\right\rangle\right|\left|e^{i(t+s) z}-e^{i(t+s) w}\right| d s d t \\
= & \int_{0}^{\infty} \int_{0}^{\infty} \mid\left\langle U_{\beta}^{*} e^{i t H \xi}\left(U_{\sigma}^{*} e^{i(t+s)\left(H_{0}-H_{\delta}^{\beta}\right)} W_{\alpha}^{*}\right) \phi, W_{\beta} e^{-i s H \xi} \psi\right| \\
& \times\left|e^{i(t+s) z}-e^{i(t+s) w}\right| d s d t .
\end{aligned}
$$


Change variables by replacing $s$ with $r=t+s$.

$$
\begin{aligned}
\mid\langle\phi,( & \left.\left.K_{1}(z)-K_{1}(w)\right) \psi\right\rangle \mid \\
< & \int_{0}^{\infty} \int_{0}^{\infty}\left|\left\langle U_{\beta}^{*} e^{i t H^{\beta}}\left(U_{\sigma}^{*} e^{i r\left(H_{0}-H^{\beta}\right)} W_{\alpha}^{*}\right) \phi, W_{\beta} e^{-i(r-t) H^{\beta}} \psi\right\rangle\right|\left|e^{i r z}-e^{i r w}\right| d t d r \\
\leqslant & \int_{0}^{\infty} \int_{0}^{\infty}\left\|U_{\beta}^{*} e^{i t H_{0}^{\beta}}\left(U_{\sigma}^{*} e^{i r\left(H_{0}-H_{0}^{\beta}\right)} W_{\alpha}^{*}\right) \phi\right\| \\
& \times\left\|W_{\beta} e^{-i(r-t) H^{\beta}} \psi\right\|\left|e^{i r z}-e^{i r w}\right| d t d r \\
\leqslant & \int_{0}^{\infty}\left(\int_{0}^{\infty}\left\|U_{\beta}^{*} e^{i t H_{0}^{\beta}}\left(U_{\sigma}^{*} e^{i r\left(H_{0}-H_{0}^{\beta}\right)} W_{\alpha}^{*}\right) \phi\right\|^{2} d t\right)^{1 / 2} \\
& \times\left(\int_{0}^{\infty}\left\|W_{\beta} e^{-i(r-t) H_{0}^{\beta}} \psi\right\|^{2} d t\right)^{1 / 2}\left|e^{i r z}-e^{i r w}\right| d r \\
\leqslant & \int_{0}^{\infty} C\left\|U_{\sigma}^{*} e^{i r\left(H_{0}-H_{0}^{\beta}\right)} W_{\alpha} \phi\right\|\|\psi\|\left|e^{i r z}-e^{i r w}\right| d r .
\end{aligned}
$$

We have used the Schwarz inequality, Hölder inequality, and Kato smoothness of $U_{\beta}^{*}$ and $W_{\beta}$ with respect to $H_{0}^{\beta}$ (see Lemma II.2).

Since $\alpha \neq \beta$ and $\beta$ has no index in common with $\sigma, \alpha$ and $\sigma$ have an index in common. Therefore, as in the proof of Lemma II.3, $\left\|U_{\sigma}^{*} e^{i r\left(H_{0}-H_{6}^{b}\right)} W_{\alpha}^{*} \phi\right\|$ is dominated by an $L^{1}$ function of $r$ which depends only on $\|\phi\|$. So, as in the proof of Lemma II.3, $\left|\left\langle\phi,\left(K_{1}(z)-K_{1}(w)\right) \psi\right\rangle\right|$ tends to zero uniformly in $\phi$ and $\psi$ as $z$ approaches $w$.

$$
\begin{aligned}
K_{2}(z)= & W_{\alpha}\left(1-P_{\alpha}\right)\left(z-H_{0}\right)^{-1} V_{\beta}\left(z-H_{0}\right)^{-1} U_{\sigma} \\
& +W_{\alpha}\left(1-P_{\alpha}\right)\left(z-H_{\alpha}\right)^{-1} U_{\alpha} K_{1}(z) .
\end{aligned}
$$

The first term is controlled by the methods used for $K_{1}(z)$. The result for $K_{1}(z)$ and Lemma V.7 control the second term.

If $\beta$ has indices in common with $\alpha$ and $\sigma$, then Lemma V.8 shows that $K_{3}(z)$ is well behaved. If $\beta$ and $\sigma$ have an index in common, but $\alpha$ and $\beta$ do not, then there are two cases:

Case 1. $\beta=\delta$ :

$$
\begin{aligned}
K_{3}(z)= & W_{\alpha}\left(1-P_{\alpha}\right)\left(z-H_{0}\right)^{-1} V_{\beta}\left(z-H_{\beta}\right)^{-1}\left(1-P_{\beta}\right) U_{\sigma} \\
& +\left(W_{\alpha}\left(1-P_{\alpha}\right)\left(z-H_{\alpha}\right)^{-1} U_{\alpha}\right) \\
& \times\left(W_{\alpha}\left(z-H_{0}\right)^{-1} V_{\beta}\left(z-H_{\beta}\right)^{-1}\left(1-P_{\beta}\right) U_{\sigma}\right) \\
= & W_{\alpha}\left(1-P_{\alpha}\right)\left[\left(z-H_{\beta}\right)^{-1}-\left(z-H_{0}\right)^{-1}\right]\left(1-P_{\beta}\right) U_{\sigma} \\
& +\left(W_{\alpha}\left(1-P_{\alpha}\right)\left(z-H_{\alpha}\right)^{-1} U_{\alpha}\right) \\
& \times\left(W_{\alpha}\left[\left(z-H_{\beta}\right)^{-1}-\left(z-H_{0}\right)^{-1}\right]\left(1-P_{\beta}\right) U_{\sigma}\right) .
\end{aligned}
$$


Since $\beta \neq \sigma$ and $\alpha$ has no index in common with $\beta, \alpha$ and $\sigma$ have an index in common. Lemmas V.6 and V.8 therefore apply to the terms which appear in the last expression.

Case 2. $\delta \neq \beta$ :

$$
\begin{aligned}
K_{3}(z) & =W_{\alpha}\left(1-P_{\alpha}\right)\left(z-H_{\alpha}\right)^{-1} V_{\beta}\left(z-H_{0}\right)^{-1}\left(1-P_{\delta}\right) U_{\sigma} \\
& +\left(W_{\alpha}\left(1-P_{\alpha}\right)\left(z-H_{\alpha}\right)^{-1} V_{\beta}\left(z-H_{0}\right)^{-1} U_{\delta}\right)\left(W_{\delta}\left(z-H_{\delta}\right)^{-1}\left(1-P_{\delta}\right) U_{\sigma}\right)
\end{aligned}
$$

The methods used to control $K_{2}(z)$ apply to the first term. The first factor of the second term has the same form as $K_{2}(z)$. Lemma V.8 applies to the final factor, since $\delta$ and $\sigma$ are assumed to have an index in common.

If $\beta$ has no indices in common with $\sigma$, then there are again two cases.

Case 3. $\beta=\delta$ : Since $\beta$ and $\sigma$ have no common index and $\alpha \neq \beta, \alpha$ and $\sigma$ must have a common index. Apply the methods of Case 1 .

Case 4. $\beta \neq \delta$ : Since $\beta$ and $\sigma$ have no index in common and $\beta \neq \delta, \delta$ and $\sigma$ have a common index. Apply the methods of Case 2.

To show $K_{4}(z)$ and $K_{5}(z)$ are well behaved, first notice that $\left(1+x_{\alpha}^{2}+\right.$ $\left.x_{\beta}^{2}\right)^{\delta / 2} W_{\beta} P_{D}\left(1+x_{\alpha}^{2}+x_{\beta}^{2}\right)^{\delta / 2}$ is bounded (by Corollary V.5). Moreover, $\sigma$ must have an index in common with $\alpha$ or $\beta$. Using this information, $K_{4}(z)$ and $K_{5}(z)$ may be rewritten as products of factors which may be controlled by the methods of Lemmas V.6 and V.8, when $U_{\beta}$ is bounded. A density argument then shows that the result holds for general $U_{\beta}$.

REMARK. Let $\xi$ be the coordinate from the center of mass of particles $i$ and $j$ to particle $k$. With the inner product on $\mathbf{R}^{3 m}$ determined by the masses, $\xi$ and $x_{i j}$ are orthogonal. However, $\xi$ is not orthogonal to any other $x_{\alpha}$.

Lemma V.10. Assume Hypotheses (1)-(3), and (5). Let $i, j$, and $k$ be distinct indices, and choose pairs $\alpha \neq(i, j)$ and $\beta \neq(i, j)$, so that $\alpha$ and $\beta$ have at least one common index. The following are well behaved:

$$
K_{1}(z)=W_{\alpha}\left(z-H_{i j}\right)^{-1} P_{i j} \rho_{i j, k}
$$

and

$$
K_{2}(z)=W_{\alpha}\left(z-H_{i j}\right)^{-1} P_{i j} U_{\beta}
$$

Proof. Notice first that $\left(z-H_{i j}\right)^{-1} P_{i j}$ equals $P_{i j}$ times a multiplication operator in the momentum representation. This fact, together with the above remark, show that $K_{1}(z)$ may be controlled by the methods of Lemma II.3 or Lemma V.6. These methods apply to $K_{2}(z)$ if $\beta$ has no index in common with $(i, j)$.

If $\beta$ has an index in common with $(i, j)$, then we may assume $\beta=(i, k) . K_{2}(z)$ may then be written as $K_{2}(z)=K_{1}(z) \rho_{i j, k}^{-1} P_{i j} U_{i k}$. Since $K_{1}(z)$ is well behaved, Lemma V.4 shows that $K_{2}(z)$ is well behaved. 
Lemma V.11. Assume Hypotheses (1)-(3), and (5). Let $i, j$, and $k$ be distinct indices. Let $\alpha=(i, j),(i, k)$, or $(j, k)$. Choose l such that $\alpha \cup\{l\}=\{i, j, k\}$. The following are well behaved:

$$
\begin{aligned}
& K_{1}(z)=\rho_{i j, k}^{-1} P_{i j} V_{i k}\left(z-H_{\alpha}\right)^{-1} P_{\alpha} \rho_{\alpha, l}, \\
& K_{2}(z)=\rho_{i j, k}^{-1} P_{i j} V_{i k}\left(1-P_{i j k}\right)\left(z-H_{\alpha}\right)^{-1} P_{\alpha} \rho_{\alpha, l}, \\
& K_{3}(z)=\rho_{i j, k}^{-1} P_{i j} V_{i k}\left(z-H_{0}\right)^{-1} P_{\alpha} \rho_{\alpha, l}, \\
& K_{4}(z)=\rho_{i j, k}^{-1} P_{i j} V_{i k}\left(1-P_{i j k}\right)\left(z-H_{0}\right)^{-1} P_{\alpha} \rho_{\alpha, l} \cdot
\end{aligned}
$$

Proof. If $\alpha \neq(i, k)$, then

$$
K_{1}(z)=\left(\rho_{i j, k}^{-1} P_{i j} U_{i k}\right)\left(W_{i k}\left(z-H_{\alpha}\right)^{-1} P_{\alpha} \rho_{\alpha, l}\right) .
$$

Apply Lemmas V.4 and V.10.

If $\alpha=(i, k)$, then $\left(z-H_{\alpha}\right)^{-1} P_{\alpha}$ equals $P_{\alpha}$ times a multiplication operator in the momentum representation. This operator $\left(z-K_{\alpha}-E_{\alpha}\right)^{-1}$ commutes with $W_{i k}$, as does $\rho_{\alpha, l}$. Therefore,

$$
K_{1}(z)=\left(\rho_{i j, k}^{-1} P_{i j} U_{i k}\left(1+x_{i j}^{2}\right)^{\gamma / 2}\right)\left(\left(1+x_{i j}^{2}\right)^{-\gamma / 2}\left(z-K_{\alpha}-E_{\alpha}\right)^{-1} \rho_{\alpha, l}\right)\left(W_{i k} P_{\alpha}\right) .
$$

Lemma V.4 bounds the first factor. The proof of Lemma II.3 controls the second. Corollary V.5 bounds the final factor.

$$
\begin{aligned}
K_{2}(z) & =K_{1}(z)-\rho_{i j, k}^{-1} P_{i j} V_{i k} P_{i j k}\left(z-H_{\alpha}\right)^{-1} P_{\alpha} \rho_{\alpha, l} \\
& =K_{1}(z)-\left(\rho_{i j, k}^{-1} P_{i j} U_{i k}\right)\left(W_{i k} P_{i j k} \rho_{\alpha, l}^{-1}\right)\left(\rho_{\alpha, l}\left(z-H_{\alpha}\right)^{-1} P_{\alpha} \rho_{\alpha, l}\right) .
\end{aligned}
$$

Lemma V.4 bounds the first factor; Corollary V.5 bounds the second; and the proof of Lemma II.3 controls the third factor.

Proofs for $K_{3}(z)$ and $K_{4}(z)$ are similar.

LEMma V.12. Assume Hypotheses (1)-(3), and (5). Let $\alpha, \beta, \delta$, and $\sigma$ be pairs, with $\alpha \neq \beta$ and $\beta \neq \sigma$. Let $k$ be any index not contained in the pair $\delta$. Let $D$ be any cluster decomposition, such that the indices of $\alpha$ belong to the same cluster and the indices of $\beta$ belong to the same cluster. The following are well behaved:

$$
\begin{aligned}
& K_{1}(z)=W_{\alpha}\left(1-P_{\alpha}\right)\left(z-H_{\alpha}\right)^{-1} V_{\beta}\left(z-H_{\delta}\right)^{-1} P_{\delta} \rho_{\delta, k}, \\
& K_{2}(z)=W_{\alpha}\left(1-P_{\alpha}\right)\left(z-H_{\alpha}\right)^{-1} V_{\beta}\left(z-H_{\delta}\right)^{-1} P_{\delta} U_{\sigma}, \\
& K_{3}(z)=W_{\alpha}\left(1-P_{\alpha}\right)\left(z-H_{\alpha}\right)^{-1} V_{\beta}\left(1-P_{D}\right)\left(z-H_{\delta}\right)^{-1} P_{\delta} \rho_{\delta, k}, \\
& K_{4}(z)=W_{\alpha}\left(1-P_{\alpha}\right)\left(z-H_{\alpha}\right)^{-1} V_{\beta}\left(1-P_{D}\right)\left(z-H_{\delta}\right)^{-1} P_{\delta} U_{\sigma}, \\
& K_{5}(z)=W_{\alpha}\left(1-P_{\alpha}\right)\left(z-H_{\alpha}\right)^{-1} V_{\beta}\left(1-P_{D}\right)\left(z-H_{0}\right)^{-1} P_{\delta} \rho_{\delta, k} .
\end{aligned}
$$

Proof. We consider only $K_{1}(z)$. The others may be treated in a similar fashion. There are three cases. 
Case $1 . \beta=\delta$ :

$$
\begin{aligned}
K_{1}(z)= & W_{\alpha}\left(1-P_{\alpha}\right)\left(z-H_{0}\right)^{-1} V_{\beta}\left(z-H_{\beta}\right)^{-1} P_{\beta} \rho_{\beta, k} \\
& +\left(W_{\alpha}\left(1-P_{\alpha}\right)\left(z-H_{\alpha}\right)^{-1} U_{\alpha}\right)\left(W_{\alpha}\left(z-H_{0}\right)^{-1} V_{\beta}\left(z-H_{\beta}\right)^{-1} P_{\beta} \rho_{\beta, k}\right) \\
= & W_{\alpha}\left(1-P_{\alpha}\right)\left[\left(z-H_{\beta}\right)^{-1}-\left(z-H_{0}\right)^{-1}\right] P_{\beta} \rho_{\beta, k} \\
& +\left(W_{\alpha}\left(1-P_{\alpha}\right)\left(z-H_{\alpha}\right)^{-1} U_{\alpha}\right)\left(W_{\alpha}\left[\left(z-H_{\beta}\right)^{-1}-\left(z-H_{0}\right)^{-1}\right] P_{\beta} \rho_{\beta, k}\right) .
\end{aligned}
$$

Apply Lemmas II.3, V.8, V.10, and the remark preceding Lemma V.10.

Case $2 . \beta \neq \delta ; \alpha$ and $\beta$ have a common index.

$$
K_{1}(z)=\left(W_{\alpha}\left(1-P_{\alpha}\right)\left(z-H_{\alpha}\right)^{-1} U_{\beta}\right)\left(W_{\beta}\left(z-H_{\delta}\right)^{-1} P_{\delta} \rho_{\delta, k}\right) .
$$

Apply Lemmas V.8 and V.10.

Case 3. $\beta \neq \delta ; \alpha$ and $\beta$ have no common index.

$$
\begin{aligned}
K_{1}(z)= & W_{\alpha}\left(1-P_{\alpha}\right)\left(z-H_{0}\right)^{-1} V_{\beta}\left(z-H_{\delta}\right)^{-1} P_{\delta} \rho_{\delta, k} \\
& +\left(W_{\alpha}\left(1-P_{\alpha}\right)\left(z-H_{\alpha}\right)^{-1} U_{\alpha}\right)\left(W_{\alpha}\left(z-H_{0}\right)^{-1} V_{\beta}\left(z-H_{\delta}\right)^{-1} P_{\delta} \rho_{\delta, k}\right) .
\end{aligned}
$$

Proceeding as in Case 1, all the results may be proved, except for norm continuity of the boundary values. However, a density argument shows that it suffices to prove norm continuity under the additional assumption that $V_{\beta}$ is bounded.

In that case,

$$
\begin{aligned}
K_{1}(z) & =\left(W_{\alpha}\left(1-P_{\alpha}\right)\left(z-H_{\alpha}\right)^{-1}\left(1+x_{\delta}^{2}\right)^{-\gamma / 2}\right) \\
& \times\left(V_{\beta}\left(1+x_{\delta}^{2}\right)^{\gamma / 2} P_{\delta}\left(z-H_{\delta}\right)^{-1} \rho_{\delta, l}\right) .
\end{aligned}
$$

Since $\alpha$ and $\beta$ have no common index and $\beta \neq \delta, \alpha$ and $\delta$ have a common index. So, Lemma V.6 applies to the first factor. Lemma V.10 and Theorem II.9 show that the second factor is well behaved.

Lemma V.13. Assume Hypotheses (1)-(3). If $N=3$, then $M(z)$ is well behaved and $(M(z))^{2}$ is compact.

Proof. $M(z)$ is well behaved by Lemmas V.4, V.8, V.11, and V.12. $W_{i j}\left(1-P_{i j}\right)(z$ $\left.-H_{i j}\right)^{-1} U_{i k}$ is a norm convergent sum of connected graphs, when $\operatorname{Re} z$ is large negative. Standard methods (e.g. Ginibre and Moulin [7]) show that each term is compact. Similarly,

$$
W_{i j}\left(1-P_{i j}\right)\left(z-H_{i j}\right)^{-1} V_{i k}\left(z-H_{i k}\right)^{-1} P_{i k} \rho_{i k, j}
$$

and

$$
\rho_{i j, k}^{-1} P_{i j} V_{i k}\left(z-H_{i k}\right)^{-1} P_{i k} \rho_{i k, j}
$$

are compact for large negative $\operatorname{Re} z$. Since these operator valued functions are well behaved, compactness holds for all $z$.

The entries of $(M(z))^{2}$ are sums of terms, each of which contains one of these compact factors. Thus $(M(z))^{2}$ is compact. 
Lemma V.14. Assume Hypotheses (1)-(3), and (5). Let $i, j$, and $k$ be distinct indices, and let $\alpha$ and $\beta$ be distinct pairs. The following are well behaved:

$$
\begin{aligned}
& K_{1}(z)=\rho_{i j, k}^{-1} P_{i j} V_{i k}\left(z-H_{0}\right)^{-1} U_{\beta}, \\
& K_{2}(z)=\rho_{i j, k}^{-1} P_{i j} V_{i k}\left(1-P_{i j k}\right)\left(z-H_{0}\right)^{-1} U_{\beta}, \\
& K_{3}(z)=\rho_{i j, k}^{-1} P_{i j} V_{i k}\left(1-P_{\alpha}\right)\left(z-H_{\alpha}\right)^{-1} U_{\beta}, \\
& K_{4}(z)=\rho_{i j, k}^{-1} P_{i j} V_{i k} P_{\alpha}\left(z-H_{\alpha}\right)^{-1} U_{\beta}, \\
& K_{5}(z)=\rho_{i j, k}^{-1} P_{i j} V_{i k}\left(1-P_{i j k}\right)\left(1-P_{\alpha}\right)\left(z-H_{\alpha}\right)^{-1} U_{\beta}, \\
& K_{6}(z)=\rho_{i j, k}^{-1} P_{i j} V_{i k}\left(1-P_{i j k}\right) P_{\alpha}\left(z-H_{\alpha}\right)^{-1} U_{\beta} .
\end{aligned}
$$

Proof. If $\beta$ and $(i, k)$ have an index in common, $K_{1}(z)$ is well behaved by Lemmas V.4 and V.6. If $\beta$ and $(i, k)$ have no common index, then Lemmas II.3 and V.4 yield the results, except for the norm continuity of the boundary values. Given this, we need only prove the norm continuity when $V_{i k}$ is bounded. In that case,

$$
K_{1}(z)=\left(\rho_{i j, k}^{-1} P_{i j} V_{i k}\left(1+x_{i j}^{2}\right)^{\gamma / 2}\right)\left(\left(1+x_{i j}^{2}\right)^{-\gamma / 2}\left(z-H_{0}\right)^{-1} U_{\beta}\right)
$$

Since $\beta$ and $(i, j)$ must have a common index, Lemma II.3 controls the boundary values of the second factor. Lemma V.4 bounds the first.

Proofs for the other operators are similar.

Proposition V.15. Assume Hypotheses (1)-(3), and (5). Let $i, j, k$, and l be distinct indices. Let $n=i, j$, or $k$. Then

$$
K_{1}(z)=W_{i j}\left(1-P_{i j}\right)\left(z-H_{i j}\right)^{-1}\left(V_{i k}+V_{j k}\right)\left(1-P_{i j k}\right)\left(z-H_{i j k}\right)^{-1} U_{n l}
$$

is well behaved and compact. Moreover

$$
K_{2}(z)=W_{i j}\left(1-P_{i j}\right)\left(z-H_{i j}\right)^{-1}\left(V_{i k}+V_{j k}\right)\left(1-P_{i j k}\right)\left(z-H_{i j k}\right)^{-1}
$$

is compact and well behaved from $L_{\delta}^{2}\left(\mathbf{R}^{3 m}\right)$ to $L^{2}\left(\mathbf{R}^{3 m}\right)$, when $\delta>1$.

Proof. Consider only $K_{1}(z)$; proof for $K_{2}(z)$ is similar.

Using equation (IV.6), we have $K_{1}(z)=\mathrm{I}(z)+\mathrm{II}(z)+\mathrm{III}(z)$, where

$$
\begin{aligned}
\mathrm{I}(z)= & W_{i j}\left(1-P_{i j}\right)\left(z-H_{i j}\right)^{-1}\left(V_{i k}+V_{j k}\right)\left(1-P_{i j k}\right)\left(z-H_{0}\right)^{-1} U_{n l}, \\
\mathrm{II}(z)= & W_{i j}\left(1-P_{i j}\right)\left(z-H_{i j}\right)^{-1}\left(V_{i k}+V_{j k}\right)\left(1-P_{i j k}\right) A(z) C(z) U_{n l}, \\
\mathrm{III}(z)= & W_{i j}\left(1-P_{i j}\right)\left(z-H_{i j}\right)^{-1}\left(V_{i k}+V_{j k}\right)\left(1-P_{i j k}\right) A(z)(1-M(z))^{-1} \\
& \times M(z) C(z) U_{n l} .
\end{aligned}
$$

Lemma V.9 shows $\mathrm{I}(z)$ is well behaved.

$A(z) C(z)$ is a sum of terms, each of which contains only one resolvent. We substitute this sum in $\operatorname{II}(z)$. II $(z)$ then becomes a sum of terms which are well behaved by Lemmas V.9 and V.12.

$W_{i j}\left(1-P_{i j}\right)\left(z-H_{i j}\right)^{-1}\left(V_{i k}+V_{j k}\right)\left(1-P_{i j k}\right) A(z)$ is well behaved by Lemmas V.9 and V.12. $M(z)$ is well behaved by Lemmas V.4, V.8, V.11, and V.12. $M(z) C(z) U_{n l}$ 
is well behaved by Lemmas V.9, V.12, and V.14. So, $\operatorname{III}(z)$ is well behaved if the factor $\left(1-P_{i j k}\right)$ in the operator

cancels the singularities of $(1-M(z))^{-1}$.

$$
W_{i j}\left(1-P_{i j}\right)\left(z-H_{i j}\right)^{-1}\left(V_{i k}+V_{j k}\right)\left(1-P_{i j k}\right) A(z)
$$

To prove this, we mimic part of the proof of Lemma V.7. $\mathcal{H}$ is a direct integral of spaces for the three body problem determined by $(i, j, k) . A(z), M(z)$, and $C(z)$ are fibered under this decomposition, and we consider their restrictions (which we also denote by $A(z), M(z)$, and $C(z))$ to the individual fibers.

Let $z_{0}$ be a point at which $(1-M(z))^{-1}$ is singular on some fixed fiber. Hypothesis (3) and Lemma II.11 show that $(1-M(z, \theta))^{-1}$ is singular at $z_{0}$ for some $\theta$ with $z_{0} \notin \sigma_{\text {ess }}(H(\theta))$ and $|\operatorname{Im} \theta|$ arbitrarily close to $0 .(1-M(z, \theta))^{-1}$ may be written as a Laurent series $\Sigma_{k=-n}^{\infty}\left(z-z_{0}\right)^{k} L_{k}$ for $z$ near $z_{0}$. Mimicking the proof of Lemma V.7, we see that $L_{-n} C\left(z_{0}, \theta\right) \neq 0$ and that no $\phi \in \operatorname{Ran} L_{-n}$ has $M\left(z_{0}, \theta\right) \phi=0$. By using $G_{\alpha}=G_{0}+G_{\alpha} V_{\alpha} G_{0}$, we see that $A\left(z_{0}, \theta\right) \psi=0$ implies $M\left(z_{0}, \theta\right) \psi=0$. Putting these facts together, $A\left(z_{0}, \theta\right) L_{-n} C\left(z_{0}, \theta\right) \neq 0$. Equation (IV.6) shows that $(z-H(\theta))^{-1}$ has a pole of order $n$ at $z_{0}$. Selfadjointness of $H$ and standard dilation analyticity arguments show $n=1$. The rest of the argument of Lemma V.7 now goes through, since equation (V.1) shows $(1-P) A\left(z_{0}\right)=$ $A\left(z_{0}\right)(1-Q)$, where $Q$ projects onto the eigenvalue 1 eigenvectors of $M\left(z_{0}\right)$. Thus $\left(1-P_{i j k}\right)$ cancels the singularities of $(1-M(z))^{-1}$, and $\operatorname{III}(z)$ is well behaved. Therefore, $K_{1}(z)$ is well behaved.

It suffices to prove compactness at large negative $\operatorname{Re} z$. Write $K_{1}(z)$ as:

$$
\begin{aligned}
& W_{i j}\left(z-H_{i j}\right)^{-1}\left(V_{i k}+V_{j k}\right)\left(z-H_{i j k}\right)^{-1} U_{n l} \\
& \quad-W_{i j} P_{i j}\left(z-H_{i j}\right)^{-1}\left(V_{i k}+V_{j k}\right)\left(z-H_{i j k}\right)^{-1} U_{n l} \\
& \quad-W_{i j}\left(z-H_{i j}\right)^{-1}\left(V_{i k}+V_{j k}\right)\left(z-H_{i j k}\right)^{-1} P_{i j k} U_{n l} \\
& \quad+W_{i j} P_{i j}\left(z-H_{i j}\right)^{-1}\left(V_{i k}+V_{j k}\right)\left(z-H_{i j k}\right)^{-1} P_{i j k} U_{n l}
\end{aligned}
$$

Expand the resolvent factors as sums of graphs. Replace each $W_{i j} P_{i j}$ by

$$
\left(W_{i j} P_{i j}\left(1+x_{i j}^{2}\right)\right)\left(1+x_{i j}^{2}\right)^{-1}
$$

and each $P_{i j k} U_{n l}$ by

$$
\left(1+x_{i j}^{2}+x_{i k}^{2}+x_{j k}^{2}\right)^{-1}\left(\left(1+x_{i j}^{2}+x_{i k}^{2}+x_{j k}^{2}\right) P_{i j k} U_{n l}\right)
$$

Then apply Corollary V.5 and any of the usual compactness proofs to each term.

Proposition V.16. Assume Hypotheses (1)-(3), and (5). Let $i, j, k$, and l be distinct indices, and let $n=i, j$, or $k$.

$$
K_{1}(z)=\rho_{i j, k}^{-1} P_{i j}\left(V_{i k}+V_{j k}\right)\left(1-P_{i j k}\right)\left(z-H_{i j k}\right)^{-1} U_{n l}
$$

is compact and well behaved.

$$
K_{2}(z)=\rho_{i j, k}^{-1} P_{i j}\left(V_{i k}+V_{j k}\right)\left(1-P_{i j k}\right)\left(z-H_{i j k}\right)^{-1}
$$

is compact and well behaved from $L_{\delta}^{2}\left(\mathbf{R}^{3 m}\right)$ to $L^{2}\left(\mathbf{R}^{3 m}\right)$ whenever $\delta>1$. 
Proof. Consider only $K_{1}(z)$; the proof for $K_{2}(z)$ is similar.

Using equation (IV.6), we have $K_{1}(z)=\mathrm{I}(z)+\mathrm{II}(z)+\mathrm{III}(z)$, where

$$
\begin{aligned}
\mathrm{I}(z) & =\rho_{i j, k}^{-1} P_{i j}\left(V_{i k}+V_{j k}\right)\left(1-P_{i j k}\right)\left(z-H_{0}\right)^{-1} U_{n l}, \\
\mathrm{II}(z) & =\rho_{i j, k}^{-1} P_{i j}\left(V_{i k}+V_{j k}\right)\left(1-P_{i j k}\right) A(z) C(z) U_{n l}, \\
\mathrm{III}(z) & =\rho_{i j, k}^{-1} P_{i j}\left(V_{i k}+V_{j k}\right)\left(1-P_{i j k}\right) A(z)(1-M(z))^{-1} M(z) C(z) U_{n l} .
\end{aligned}
$$

Lemma V.14 shows that $\mathrm{I}(z)$ and $\mathrm{II}(z)$ are well behaved. $M(z)$ is well behaved by Lemmas V.4, V.8, V.12, and V.14. $M(z) C(z) U_{n l}$ is well behaved by Lemmas V.9, V.12, and V.14. Lemmas V.11 and V.14 control $\rho_{i j, k}^{-1} P_{i j}\left(V_{i k}+V_{j k}\right)\left(1-P_{i j k}\right) A(z)$.

As in Proposition V.15, $\left(1-P_{i j k}\right)$ removes the singularities of $(1-M(z))^{-1}$. So, $K_{1}(z)$ is well behaved.

It suffices to prove compactness for large negative $\operatorname{Re} z$. Furthermore, a density argument shows that we may assume all $U$ 's and $W$ 's lie in $\delta\left(\mathbf{R}^{m}\right)$.

Under this assumption,

$$
\begin{aligned}
\rho_{i j, k}^{-1} P_{i j} & \left(V_{i k}+V_{j k}\right) P_{i j k}\left(z-H_{i j k}\right)^{-1} U_{n l} \\
& =\left(\rho_{i j, k}^{-1} P_{i j}\left(V_{i k}+V_{j k}\right)\right)\left(P_{i j k}\left(z-H_{i j k}\right)^{-1} \rho_{i j k}\right)\left(\rho_{i j k}^{-1} P_{i j k} U_{n l}\right) .
\end{aligned}
$$

Lemma V.4 bounds the initial and final factors. The middle factor equals $P_{i j k}(z-$ $\left.K_{i j k}-E_{i j k}\right)^{-1} \rho_{i j k}$, where $K_{i j k}$ is the kinetic energy associated with the coordinate from the center of mass of particles $i, j$, and $k$ to particle $l . P_{i j k}$ is (compact) $\otimes 1$. $\left(z-K_{i j k}-E_{i j k}\right)^{-1} \rho_{i j k}$ is $1 \otimes$ (compact). So, the middle term is compact.

Hence, it suffices to prove $\rho_{i j, k}^{-1} P_{i j}\left(V_{i k}+V_{j k}\right)\left(z-H_{i j k}\right)^{-1} U_{n l}$ is compact when the $U$ 's and $W$ 's belong to $\delta\left(\mathbf{R}^{m}\right)$. Lemma V.4 shows that it suffices to prove that $\left(1+x_{i j}^{2}\right)^{-\alpha}\left(W_{i k}+W_{j k}\right)\left(z-H_{i j k}\right)^{-1} U_{n l}$ is compact. We expand $\left(z-H_{i j k}\right)^{-1}$ as a sum of graphs. The only graphs for which compactness could fail are those which do not connect $i$ and $j$. It therefore suffices to prove the compactness of $(1+$ $\left.x_{i j}^{2}\right)^{-\alpha}\left(W_{i k}\left(z-H_{i k}\right)^{-1}+W_{j k}\left(z-H_{j k}\right)^{-1}\right) U_{n l}$.

Expand $\left(1+x_{i j}^{2}\right)^{-\alpha} W_{i k}\left(z-H_{i k}\right)^{-1} U_{n l}$ as a sum of graphs. Use coordinates $x_{i j}$, $x_{i k}$, and $x_{n l}$, with conjugate momenta $p_{i j}, p_{i k}$, and $p_{n l}$, respectively. Following Reed and Simon [24, p. 128], write out the integral kernel for each graph in momentum representation. If $z$ is real and large negative, then the replacement of each factor $\left(z-H_{0}\right)^{-1}$ by $\left(z-p_{i j}^{2}-p_{i k}^{2}-p_{n l}^{2}\right)^{-1}$ will increase the absolute value of the integral kernel at every point. The new integral kernel is square integrable, so the original one is, also. Consequently, each graph is Hilbert-Schmidt.

The proof for $\left(1+x_{i j}^{2}\right)^{-\alpha} W_{j k}\left(z-H_{j k}\right)^{-1} U_{n l}$ is similar.

Proposition V.17. Assume Hypotheses (1)-(3), and (5). Let $i, j, k$, and l be distinct indices; let $n=i, j$, or $k$; let $p$ be any index not equal to $n$ or $l$; and let $\alpha$ be the pair not containing $n$ or $l$. The following are well behaved and compact:

$$
\begin{aligned}
K_{1}(z)= & W_{i j}\left(1-P_{i j}\right)\left(z-H_{i j}\right)^{-1}\left(V_{i k}+V_{j k}\right)\left(1-P_{i j k}\right)\left(z-H_{i j k}\right)^{-1} \\
& \times V_{n l}\left(z-H_{n l}\right)^{-1} P_{n l} \rho_{n l, p}
\end{aligned}
$$


and

$$
\begin{aligned}
K_{2}(z)= & W_{i j}\left(1-P_{i j}\right)\left(z-H_{i j}\right)^{-1}\left(V_{i k}+V_{j k}\right)\left(1-P_{i j k}\right)\left(z-H_{i j k}\right)^{-1} \\
& \times V_{n l}\left(z-H_{n l}\right)^{-1} P_{n l} U_{\alpha} .
\end{aligned}
$$

Proof. Consider only $K_{1}(z)$; the proof for $K_{2}(z)$ is essentially identical.

For large negative $\operatorname{Re} z, W_{n l} P_{n l}\left(z-H_{n l}\right)^{-1} \rho_{n l, p}$ is bounded, so compactness follows from Proposition V.15. Similarly, it is clear that $\left\|K_{1}(z)\right\|$ tends to zero as $\operatorname{Re} z \rightarrow-\infty$. It is therefore sufficient to prove analyticity and the norm continuity of the boundary values.

$$
\begin{aligned}
K_{1}(z)=\mathrm{I}(z)+ & \mathrm{II}(z)+\mathrm{III}(z), \text { where } \\
\mathrm{I}(z)= & W_{i j}\left(1-P_{i j}\right)\left(z-H_{i j}\right)^{-1}\left(V_{i k}+V_{j k}\right)\left(1-P_{i j k}\right)\left(z-H_{0}\right)^{-1} \\
& \times V_{n l}\left(z-H_{n l}\right)^{-1} P_{n l} \rho_{n l p}, \\
\mathrm{II}(z)= & W_{i j}\left(1-P_{i j}\right)\left(z-H_{i j}\right)^{-1}\left(V_{i k}+V_{j k}\right)\left(1-P_{i j k}\right) A(z) C(z) \\
& \times V_{n l}\left(z-H_{n l}\right)^{-1} P_{n l} \rho_{n l p}, \\
\operatorname{III}(z)= & W_{i j}\left(1-P_{i j}\right)\left(z-H_{i j}\right)^{-1}\left(V_{i k}+V_{j k}\right)\left(1-P_{i j k}\right) A(z) \\
& \times(1-M(z))^{-1} M(z) C(z) V_{n l}\left(z-H_{n l}\right)^{-1} P_{n l} \rho_{n l p} .
\end{aligned}
$$

Lemma V.12 controls $\mathrm{I}(z)$ after the factor $\left(z-H_{0}\right)^{-1} V_{n l}\left(z-H_{n l}\right)^{-1}$ is replaced by $\left[\left(z-H_{n l}\right)^{-1}-\left(z-H_{0}\right)^{-1}\right]$.

Since

$$
\begin{aligned}
A(z) C(z)= & \left(z-H_{i j}\right)^{-1}+\left(z-H_{i k}\right)^{-1}+\left(z-H_{j k}\right)^{-1}-3\left(z-H_{0}\right)^{-1} \\
= & {\left[\left(z-H_{i j}\right)^{-1} V_{i j}+\left(z-H_{i k}\right)^{-1} V_{i k}+\left(z-H_{j k}\right)^{-1} V_{j k}\right]\left(z-H_{0}\right)^{-1}, } \\
\mathrm{II}(z)= & \sum_{\beta=\underset{(j j),(i k),}{(j k)}} W_{i j}\left(1-P_{i j}\right)\left(z-H_{i j}\right)^{-1}\left(V_{i k}+V_{j k}\right) \\
& \times\left(1-P_{i j k}\right)\left(z-H_{\beta}\right)^{-1} V_{\beta}\left[\left(z-H_{n l}\right)^{-1}-\left(z-H_{0}\right)^{-1}\right] P_{n l} \rho_{n l p} \\
= & \sum_{\beta} W_{i j}\left(1-P_{i j}\right)\left(z-H_{i j}\right)^{-1}\left(V_{i k}+V_{j k}\right)\left(1-P_{i j k}\right)\left(z-H_{\beta}\right)^{-1} V_{\beta} \\
& \times\left(z-H_{n l}\right)^{-1} P_{n l} \rho_{n l p} \\
& -\sum_{\beta} W_{i j}\left(1-P_{i j}\right)\left(z-H_{i j}\right)^{-1}\left(V_{i k}+V_{j k}\right)\left(1-P_{i j k}\right) \\
& \times\left[\left(z-H_{\beta}\right)^{-1}-\left(z-H_{0}\right)^{-1}\right] P_{n l} \rho_{n l p} .
\end{aligned}
$$

Using the remark before Lemma V.10, Lemma II.3, and Theorem II.9, we see that $\left(1+x_{n l}^{2}\right)^{\delta} W_{\beta}\left(z-H_{n l}\right)^{-1} P_{n l} \rho_{n l, p}$ is well behaved for each $\beta=(i, j)(i, k)$, or $(j, k)$. So, to control the first sum, it is sufficient to control

$$
W_{i j}\left(1-P_{i j}\right)\left(z-H_{i j}\right)^{-1}\left(V_{i k}+V_{j k}\right)\left(1-P_{i j k}\right)\left(z-H_{\beta}\right)^{-1} U_{\beta}\left(1+x_{n l}^{2}\right)^{-\delta} .
$$


This equals

$$
\begin{aligned}
& W_{i j}\left(1-P_{i j}\right)\left(z-H_{i j}\right)^{-1}\left(V_{i k}+V_{j k}\right)\left(1-P_{i j k}\right)\left(1-P_{\beta}\right) \\
& \quad \times\left(z-H_{\beta}\right)^{-1} U_{\beta}\left(1+x_{n l}^{2}\right)^{-\delta}+W_{i j}\left(1-P_{i j}\right)\left(z-H_{i j}\right)^{-1}\left(V_{i k}+V_{j k}\right) \\
& \quad \times\left(1-P_{i j k}\right) P_{\beta}\left(z-H_{\beta}\right)^{-1} U_{\beta}\left(1+x_{n l}^{2}\right)^{-\delta} .
\end{aligned}
$$

Lemma V.9 controls the first of these terms. The second is well behaved by Corollary V.5 and Lemmas V.4 and V.12.

The second sum in the last expression for $\mathrm{II}(z)$ is controlled by Lemmas V.9 and V.12.

Next consider III $(z)$.

$$
W_{i j}\left(1-P_{i j}\right)\left(z-H_{i j}\right)^{-1}\left(V_{i k}+V_{j k}\right)\left(1-P_{i j k}\right) A(z)(1-M(z))^{-1}
$$

has been studied in the proof of Proposition V.15. So, it suffices to control $M(z) C(z) V_{n l}\left(z-H_{n l}\right)^{-1} P_{n l} \rho_{n l, p}$.

Two types of terms appear here:

$$
W_{\delta}\left(1-P_{\delta}\right)\left(z-H_{\delta}\right)^{-1} V_{\sigma}\left(z-H_{\sigma}\right)^{-1} V_{n l}\left(z-H_{n l}\right)^{-1} P_{n l} \rho_{n l p},
$$

and

$$
\rho_{\delta, q}^{-1} P_{\delta} V_{\sigma}\left(z-H_{\sigma}\right)^{-1} V_{n l}\left(z-H_{n l}\right)^{-1} P_{n l} \rho_{n l, p}
$$

where $\delta$ and $\sigma$ have a common index. The first type appears in II(z), and has been studied. The second equals

$$
\begin{aligned}
\rho_{\delta, q}^{-1} P_{\delta} V_{\sigma} & \left(1-P_{\sigma}\right)\left(z-H_{\sigma}\right)^{-1} V_{n l}\left(z-H_{n l}\right)^{-1} P_{n l} \rho_{n l p} \\
& +\rho_{\delta, q}^{-1} P_{\delta} V_{\sigma} P_{\sigma}\left(z-H_{\sigma}\right)^{-1} V_{n l}\left(z-H_{n l}\right)^{-1} P_{n l} \rho_{n l p} .
\end{aligned}
$$

The first of these is well behaved by Lemmas V.4 and V.12. The second may be rewritten as:

$$
\begin{gathered}
\left(\rho_{\delta, q}^{-1} P_{\delta} U_{\sigma}\left(1+x_{\delta}^{2}\right)^{\gamma / 2}\right)\left(W_{\sigma}\left(1+x_{\delta}^{2}\right)^{-\gamma / 2} P_{\sigma}\left(z-H_{\sigma}\right)^{-1} U_{n l}\left(1+x_{\sigma}^{2}\right)^{\gamma / 2}\right) \\
\times\left(\left(1+x_{\sigma}^{2}\right)^{-\gamma / 2} P_{n l}\left(z-H_{n l}\right)^{-1} \rho_{n l, p}\right) .
\end{gathered}
$$

Lemma V.4 applies to the first factor. Lemma V.4 and the proof of Lemma II.3 control the others.

Proposition V.18. Assume Hypotheses (1)-(3), and (5). Let $i, j, k$, and $l$ be distinct; let $n=i, j$, or $k$; let $p$ be any index not equal to $n$ or $l$; let $\alpha$ be the pair not containing $n$ or $p$. The following are well behaved and compact:

$$
\begin{aligned}
& K_{1}(z)=\rho_{i j, k}^{-1} P_{i j}\left(V_{i k}+V_{j k}\right)\left(1-P_{i j k}\right)\left(z-H_{i j k}\right)^{-1} V_{n l} P_{n l}\left(z-H_{n l}\right)^{-1} \rho_{n l, p}, \\
& K_{2}(z)=\rho_{i j, k}^{-1} P_{i j}\left(V_{i k}+V_{j k}\right)\left(1-P_{i j k}\right)\left(z-H_{i j k}\right)^{-1} V_{n l} P_{n l}\left(z-H_{n l}\right)^{-1} U_{\alpha} .
\end{aligned}
$$

Proof. The technique for extending the proof for Proposition V.16 to these operators is the same as the technique used to obtain the proof of Proposition V.17 from that of Proposition V.15. In some instances, Lemma V.14 must be used in place of Lemmas V.9 and V.12. 
Proposition V.19. Assume Hypotheses (1)-(3), and (5). Let $i, j, k$, and $l$ be distinct, and let $n=j$ or $k$. The following are compact and well behaved:

$$
\begin{aligned}
& K_{1}(z)=\rho_{i j k}^{-1} P_{i j k} V_{i l} P_{i l}\left(z-H_{i l}\right)^{-1} \rho_{i l, n}, \\
& K_{2}(z)=\rho_{i j k}^{-1} P_{i j k} V_{i l} P_{i l}\left(z-H_{i l}\right)^{-1} U_{j k}, \\
& K_{3}(z)=\rho_{i j, k l}^{-1} P_{i j, k l} V_{i l} P_{i l}\left(z-H_{i l}\right)^{-1} \rho_{i l, n}, \\
& K_{4}(z)=\rho_{i j, k l}^{-1} P_{i j, k l} V_{i l} P_{i l}\left(z-H_{i l}\right)^{-1} U_{j k} .
\end{aligned}
$$

Proof. Consider only $K_{1}(z)$; proofs for the others are similar.

$$
\begin{aligned}
K_{1}(z)= & \left(\rho_{i j k}^{-1} P_{i j k} U_{i l}\left(1+x_{i j}^{2}+x_{i k}^{2}+x_{j k}^{2}\right)^{\alpha}\right) \\
& \times\left(\left(1+x_{i j}+x_{i k}+x_{j k}\right)^{-\alpha} W_{i l} P_{i l}\left(z-H_{i l}\right)^{-1} \rho_{i l, n}\right) .
\end{aligned}
$$

Lemma V.4 bounds the first factor. $P_{i l}\left(z-H_{i l}\right)^{-1}=P_{i l}\left(z-K_{i l}-E_{i l}\right)^{-1}$, where $K_{i l}$ is a multiplication operator in the momentum representation. The methods of Lemma II.3 may be used to prove that the second factor is well behaved. For large negative $\operatorname{Re} z,\left(1+x_{i j}^{2}+x_{i k}^{2}+x_{j k}^{2}\right)^{-\alpha} W_{i l}\left(z-H_{i l}\right)^{-1}$ is compact because $\left(1+x_{i j}^{2}\right.$ $\left.+x_{i k}^{2}+x_{j k}^{2}\right)^{-\alpha} W_{i l}$ falls off in all directions in configuration space. This implies compactness.

Proposition V.20. Assume Hypotheses (1)-(3), and (5). Let $i, j, k$, and $l$ be distinct; let $n=j$ or $l$; and let $p=j$ or $k$. The following are well behaved and compact:

$$
\begin{aligned}
& K_{1}(z)=\rho_{i j k}^{-1} P_{i j k} V_{i l}\left(z-H_{i l}\right)^{-1}\left(V_{i p}+V_{l}\right)\left(z-H_{i l p}\right)^{-1} P_{i p} \rho_{i l}, \\
& K_{2}(z)=\rho_{i j k}^{-1} P_{i j k} V_{i l}\left(z-H_{i l}\right)^{-1} V_{j k}\left(z-H_{i l, j k}\right)^{-1} P_{i l, j k} \rho_{i l j k}, \\
& K_{3}(z)=\rho_{i j, k l}^{-1} P_{i j, k l} V_{i k}\left(z-H_{i k}\right)^{-1}\left(V_{i n}+V_{k n}\right)\left(z-H_{i k n}\right)^{-1} P_{i k n} \rho_{i k n}, \\
& K_{4}(z)=\rho_{i j, k l}^{-1} P_{i j, k l} V_{i k}\left(z-H_{i k}\right)^{-1} V_{j l}\left(z-H_{i k, j l}\right)^{-1} P_{i k, j l} \rho_{i k, j l} .
\end{aligned}
$$

Proof. Consider only $K_{1}(z)$; proofs for the others are similar.

$$
\begin{aligned}
K_{1}(z)= & \rho_{i j k}^{-1} P_{i j k} V_{i l}\left(z-H_{i l p}\right)^{-1} P_{i l p} \rho_{i l p} \\
& -\rho_{i j k}^{-1} P_{i j k} V_{i l}\left(1-P_{i l}\right)\left(z-H_{i l}\right)^{-1} P_{i l} \rho_{i l p} \\
& -\rho_{i j k}^{-1} P_{i j k} V_{i l} P_{i l}\left(z-H_{i l}\right)^{-1} P_{i l} \rho_{i l p} .
\end{aligned}
$$

The methods used to prove Proposition V.19 may easily be modified to control the first and last terms. The middle term may be written as:

$$
-\left(\rho_{i j k}^{-1} P_{i j k} U_{i l}\right)\left(W_{i l}\left(1-P_{i l}\right)\left(z-H_{i l}\right)^{-1}\left(1+x_{i l}^{2}\right)^{-\gamma / 2}\right)\left(\left(1+x_{i l}^{2}\right)^{\gamma / 2} P_{i l p} \rho_{i l p}\right) \text {. }
$$

Lemma V.4 bounds the first factor. Theorem II.9 bounds the third. The proof of Lemma V.7 controls the middle factor.

Given the above results, it suffices to prove compactness when $V_{i p}$ and $V_{i p}$ are bounded. In that case,

$$
K_{1}(z)=\left(\rho_{i j k}^{-1} P_{i j k} V_{i l}\left(z-H_{i l}\right)^{-1}\left(V_{i p}+V_{\varphi}\right)\right)\left(\left(z-H_{i p}\right)^{-1} P_{i l} \rho_{i l p}\right) .
$$


For large negative $\operatorname{Re} z$, the first factor is bounded. In the second,

$$
\left(z-H_{i l p}\right)^{-1} P_{i l p}=\left(z-K_{i l p}-E_{i l p}\right)^{-1} P_{i l p} .
$$

$P_{i l p}$ is (compact) $\otimes 1 ;\left(z-K_{i l p}-E_{i l p}\right)^{-1} \rho_{i l p}$ is $1 \otimes$ (compact). This implies compactness.

In order to prove certain results involving $\left(z-H_{i j, k l}\right)^{-1}$, a formula analogous to equation (IV.6) must be obtained. To motivate the formula we will use, we first give a simpler formula:

$$
\left(z-H_{i j, k l}\right)^{-1}=\left(z-H_{0}\right)^{-1}+\mathbb{Q}(z)(1-\mathscr{T}(z))^{-1} \mathcal{C}(z)
$$

where

$$
\begin{aligned}
\mathcal{Q}(z) & =\left[\begin{array}{lc}
\left(z-H_{0}\right)^{-1} U_{i j} & \left(z-H_{0}\right)^{-1} U_{k l}
\end{array}\right] \\
\mathscr{N}(z) & =\left[\begin{array}{cc}
0 & W_{i j}\left(z-H_{i j}\right)^{-1} U_{k l} \\
W_{k l}\left(z-H_{k l}\right)^{-1} U_{i j} & 0
\end{array}\right], \\
\mathcal{C}(z) & =\left[\begin{array}{c}
W_{i j}\left(z-H_{i j}\right)^{-1} \\
W_{k l}\left(z-H_{k l}\right)^{-1}
\end{array}\right] .
\end{aligned}
$$

For large negative $\operatorname{Re} z$, the inverse term may be expanded by geometric series. Then, using the diagram symbolism, the formula is easily checked. Using the uniqueness of analytic continuation, the formula is valid wherever both sides contain only bounded operators.

If the relative motion of the centers of mass of particles $i$ and $j$ and of particles $k$ and $l$ is removed, then the barely connected terms $W_{i j}\left(z-H_{i j}\right)^{-1} U_{k l}$ and $W_{k l}(z-$ $\left.H_{k l}\right)^{-1} U_{i j}$ are compact. So, the analytic Fredholm theorem shows that the inverse term of equation (V.5) exists for $z \notin \sigma_{\text {ess }}\left(H_{i j, k l}\right)$ except at those $z$ 's for which the system of equations

$$
\phi_{i j}=W_{i j}\left(z-H_{i j}\right)^{-1} U_{k l} \phi_{k l} \text { and } \phi_{k l}=W_{k l}\left(z-H_{k l}\right)^{-1} U_{i j} \phi_{i j}
$$

has a nontrivial solution. Mimicking equation (V.1), we see that this occurs only when $z$ is an eigenvalue of $H_{i j, k l}$. Thus, equation (V.5) is valid for $z \in C \backslash \sigma\left(H_{i j, k l}\right)$.

As $z$ approaches $\sigma_{\text {ess }}\left(H_{i j, k l}\right)$, various terms in equation (V.5) are singular, unless $P_{i j}=P_{k l}=0$. So, we require another formula. Because $V_{k l}$ and $P_{i j}$ commute, there is no analogue of the $\rho$ functions in this case. However, a formula very much like equation (IV.6) is valid. Let $G_{0}=\left(z-H_{0}\right)^{-1}, G_{i j}=\left(z-H_{i j}\right)^{-1}$, etc. Then,

$$
G_{i j, k l}=G_{i j}+G_{k l}-G_{0}+A(z)(1-M(z))^{-1} C(z)
$$

where $A(z), M(z)$, and $C(z)$ are given in Figure 2. 


$$
\begin{aligned}
& A(z)=\left[\begin{array}{llll}
G_{0} U_{i j} & \left(G_{i j}-G_{o}\right) P_{i j} U_{k \ell} & G_{o} U_{k \ell} & \left(G_{k \ell}-G_{o}\right) P_{k \ell} U_{i j}
\end{array}\right] \\
& M(z)=\left[\begin{array}{cccc}
0 & 0 & w_{i j}\left(1-P_{i j}\right) G_{i j} U_{k \ell} & w_{i j}\left(1-P_{i j}\right) G_{i j} V_{k \ell} P_{k \ell} G_{k \ell} U_{i j} \\
0 & 0 & P_{i j} & P_{i j} W_{k \ell} P_{k \ell} G_{k \ell} U_{i j} \\
w_{k \ell}\left(1-P_{k \ell}\right) C_{k \ell} U_{i j} & w_{k \ell}\left(1-P_{k \ell}\right) C_{k \ell} V_{i j} P_{i j} G_{i j} U_{k \ell} & 0 & 0 \\
P_{k \ell} & P_{k \ell} W_{i j} P_{i j} G_{i j} U_{k \ell} & 0 & 0
\end{array}\right] \\
& C(z)=\left[\begin{array}{l}
w_{i j}\left(1-P_{i j}\right) G_{i j} v_{k \ell} C_{k \ell} \\
P_{i j} W_{k \ell} G_{k \ell} \\
w_{k \ell}^{\prime}\left(1-P_{k \ell}\right) G_{k \ell} v_{i j} G_{i j} \\
P_{k l} W_{i j} G_{i j}
\end{array}\right]
\end{aligned}
$$

FIGURE 2

To establish the validity of equation (V.6), we expand the inverse term of equation (V.5) and insert projections $P_{i j},\left(1-P_{i j}\right), P_{k l}$ and $\left(1-P_{k l}\right)$ before the resolvent factors. We then sum the resulting series to obtain equation (V.6). This is completely analogous to the derivation of equation (IV.6) from equation (IV.4). The absence of spurious zeros for equation (V.6) is proved by mimicking the proof of Proposition V.2. Below, we will prove $M(z)$ is well behaved, and $(M(z))^{2}$ is compact (when the motion of the center of mass of particles $i$ and $j$ relative to the center of mass of particles $k$ and $l$ is removed). $A(z)$ and $C(z)$ are analytic for $z \in \mathrm{C} \backslash \sigma_{\text {ess }}\left(H_{i j, k l}\right)$, so equation (V.6) is valid for $z \notin \sigma\left(H_{i j, k l}\right)$.

In the two and three body cases (with finite masses), $M(z)$ was well behaved. We have not been able to prove this for equation (V.6) (or for the three body case with an infinite mass, in which case an equation like (V.6) is used in place of equation (IV.6)). However, $(M(z))^{2}$ is well behaved. The next few lemmas prove this.

Lemma V.21. Assume Hypotheses (1)-(3), and (5). Let $i, j, k$, and $l$ be distinct. $K(z)=W_{i j}\left(1-P_{i j}\right)\left(z-H_{i j}\right)^{-1} U_{k l}$ is analytic for $z \in \mathrm{C} \backslash \sigma_{\text {ess }}\left(H_{i j, k l}\right)$; tends to zero as $\operatorname{Re} z \rightarrow-\infty$; and has strongly continuous boundary values on $\sigma_{\text {ess }}\left(H_{i j, k l}\right)$ from above and below. If the motion of the center of mass of particles $i$ and $j$ relative to the center of mass of particles $k$ and $l$ has been removed, then $K(z)$ is compact for $z \in \mathbf{C} \backslash \sigma_{\text {ess }}\left(H_{i j, k l}\right)$.

Proof. Except for compactness, Lemma II.3 yields these results for the operator valued function $W_{i j}\left(1-P_{i j}\right)\left(z-H_{0}\right)^{-1} U_{k l}$ :

$$
\begin{aligned}
K(z)= & W_{i j}\left(1-P_{i j}\right)\left(z-H_{0}\right)^{-1} U_{k l} \\
& +\left(W_{i j}\left(1-P_{i j}\right)\left(z-H_{i j}\right)^{-1} U_{i j}\right)\left(W_{i j}\left(z-H_{0}\right)^{-1} U_{k l}\right)
\end{aligned}
$$

Lemmas II.3 and V.7 apply to the various factors here.

To prove compactness, we expand the resolvent in $K(z)$ as a sum of graphs. The standard compactness proofs apply to each term at large negative $\operatorname{Re} z$. 
Lemma V.22. Assume Hypotheses (1)-(3), and (5). Let $i, j, k$, and $l$ be distinct. $K(z)=P_{i j} W_{k l} P_{k l}\left(z-H_{k l}\right)^{-1} U_{i j}$ is well behaved. Moreover, if the relative motion of the centers of mass of cluster $\{i, j\}$ and $\{k, l\}$ is removed, then $K(z)$ is compact.

Proof. $K(z)=\left(W_{k l} P_{k l}\right)\left(P_{k l} P_{i j}\left(1+x_{i j}^{2}\right)^{\alpha}\right)\left(\left(1+x_{i j}^{2}\right)^{-\alpha}\left(z-K_{k l}-E_{k l}\right)^{-1} U_{i j}\right)$. Corollary V.5 bounds the first factor. The second is compact on $L^{2}\left(\mathbf{R}^{2 m}\right)$. Lemma II. 3 controls the third.

Lemma V.23. Assume Hypotheses (1)-(3), and (5). If the relative motion of the centers of mass of the clusters $\{i, j\}$ and $\{k, l\}$ is removed, then $M(z)$ in equation (V.6) is analytic in $\mathrm{C} \backslash \sigma_{\mathrm{ess}}\left(\boldsymbol{H}_{i j, k l}\right)$, with strongly continuous boundary values on the cut. $(M(z))^{2}$ is compact and well behaved.

Proof. All results, except for compactness and norm continuity of $(M(z))^{2}$ up to the cut, are consequences of Lemmas V.12, V.21, and V.22.

$(M(z))^{2}$ has four types of nonzero terms.

Type 1. $W_{i j}\left(1-P_{i j}\right)\left(z-H_{i j}\right)^{-1} V_{k l}\left(z-H_{k l}\right)^{-1} U_{i j}$.

Lemmas V.9 and V.12 imply the continuity. Compactness follows from Lemma V.21, since $U_{k l}\left(z-H_{k l}\right)^{-1} U_{i j}$ is bounded for large negative $\operatorname{Re} z$.

Type 2. $P_{i j} W_{k l}\left(z-H_{k l}\right)^{-1} U_{i j}$.

This equals

$$
P_{i j} W_{k l} P_{k l}\left(z-H_{k l}\right)^{-1} U_{i j}+P_{i j} W_{k l}\left(1-P_{k l}\right)\left(z-H_{k l}\right)^{-1} U_{i j}
$$

So, Lemmas V.21 and V.22 show that it suffices to prove the continuity when $W_{k l}$ is bounded. In that case,

$$
P_{i j} W_{k l}\left(z-H_{k l}\right)^{-1} U_{i j}=\left(W_{k l} P_{i j}\left(1+x_{i j}^{2}\right)^{\alpha}\right)\left(\left(1+x_{i j}^{2}\right)^{-\alpha}\left(z-H_{k l}\right)^{-1} U_{i j}\right) .
$$

The first factor is bounded. The second is well behaved by Lemma II.3.

Type 3. $W_{i j}\left(1-P_{i j}\right) V_{k l}\left(z-H_{k l}\right)^{-1} V_{i j}\left(z-H_{i j}\right) P_{i j} U_{k l}$.

$G_{i j}-G_{k l}=G_{k l}\left(V_{i j}-V_{k l}\right) G_{i j}$, so the operator in question equals

$$
\begin{aligned}
& W_{i j}\left(1-P_{i j}\right)\left(z-H_{i j}\right)^{-1} V_{k l}\left(z-H_{i j}\right)^{-1} P_{i j} U_{k l} \\
& \quad-W_{i j}\left(1-P_{i j}\right)\left(z-H_{i j}\right)^{-1} V_{k l}\left(z-H_{k l}\right)^{-1} P_{i j} U_{k l} \\
& \quad+W_{i j}\left(1-P_{i j}\right)\left(z-H_{i j}\right)^{-1} V_{k l}\left(z-H_{k l}\right)^{-1} V_{k l}\left(z-H_{i j}\right)^{-1} P_{i j} U_{k l} \cdot
\end{aligned}
$$

The first term here is zero, since the $P_{i j}$ commutes with $\left(z-H_{i j}\right)^{-1} V_{k l}\left(z-H_{i j}\right)^{-1}$. By Lemmas V.21 and V.22, it suffices to prove the continuity for the remaining terms when $V_{k l}$ and $U_{k l}$ are bounded. The second term is controlled by Lemmas V.9 and V.12 after factorization as

$$
-\left(W_{i j}\left(1-P_{i j}\right)\left(z-H_{i j}\right)^{-1} V_{k l}\left(z-H_{k l}\right)^{-1}\left(1+x_{i j}^{2}\right)^{-\alpha}\right)\left(\left(1+x_{i j}^{2}\right)^{\alpha} P_{i j} U_{k l}\right) .
$$

The third term equals

$$
\begin{gathered}
\left(W_{i j}\left(1-P_{i j}\right)\left(z-H_{i j}\right)^{-1} V_{k l}\left(z-H_{k l}\right)^{-1}\left(1+x_{i j}^{2}\right)^{-\alpha}\right) \\
\times\left(\left(1+x_{i j}^{2}\right)^{\alpha} P_{i j} U_{k l}\right)\left(W_{k l}\left(z-H_{i j}\right)^{-1} U_{k l}\right) .
\end{gathered}
$$


Lemmas V.9 and V.12 control the first factor. The second factor is bounded. The third is controlled by Lemma II.3.

Type 4. $P_{i j} W_{k l}\left(z-H_{k l}\right)^{-1} V_{i j}\left(z-H_{i j}\right)^{-1} P_{i j} U_{k l}$.

This equals

$$
\begin{aligned}
P_{i j} W_{k l}\left(1-P_{k l}\right)\left(z-H_{k l}\right)^{-1} V_{i j}\left(z-H_{i j}\right)^{-1} P_{i j} U_{k l} \\
+\left(P_{i j} W_{k l} P_{k l}\left(z-H_{k l}\right)^{-1} U_{i j}\right)\left(P_{k l} W_{i j} P_{i j}\left(z-H_{i j}\right)^{-1} U_{k l}\right) .
\end{aligned}
$$

Compactness follows from Lemmas V.21 and V.22. Lemmas V.12 and V.22 imply the norm continuity of the first and second terms, respectively.

Remark. $C(z)$ in equation (V.6) is well behaved from $L_{\delta}^{2}\left(\mathbf{R}^{2 m}\right)$ to $L^{2}\left(\mathbf{R}^{2 m}\right)$. Also,

$$
G_{i j}=G_{i j} P_{i j}+G_{0}\left(1-P_{i j}\right)+G_{0} V_{i j} G_{i j}\left(1-P_{i j}\right)
$$

and

$$
G_{k l}=G_{k l} P_{k l}+G_{0}\left(1-P_{k l}\right)+G_{0} V_{k l} G_{k l}\left(1-P_{k l}\right)
$$

These facts, together with Lemma V.23 and Theorem III.1 imply asymptotic completeness for $H_{i j, k l}$, when Hypotheses (1)-(3) hold. Much easier proofs of this fact exist, but this result is a simple by-product of our methods.

LEMMA V.24. Suppose $A(z)$ is a strongly continuous bounded function with values in the bounded operators on $\mathcal{H}$. Suppose $B(z)$ is a norm continuous function with values in the compact operators on $\mathcal{H}$. Then $A(z) B(z)$ is norm continuous and compact.

Proof. The compactness of $A(z) B(z)$ is a well-known fact.

Suppose $\varepsilon>0$ is given. Fix $z$, and let $N=\sup \|A(w)\|$. Choose a finite rank operator $C$, such that $\|B(z)-C\|<\varepsilon / 6 N$. The restriction of $A(w)$ to the range of $C$ is norm continuous. Therefore, there exists $\delta>0$, so that $|z-w|<\delta$ implies $\|(A(z)-A(w)) C\|<\varepsilon / 3 N$.

If $|z-w|<\delta$, then by the triangle inequality,

$$
\begin{aligned}
\| A(z) B(z)- & A(w) B(w) \| \\
\leqslant & \|(A(z)-A(w))(B(z)-C)\|+\|(A(z)-A(w)) C\| \\
& +\|A(w)(B(z)-B(w))\| \\
< & 2 N \varepsilon / 6 N+\varepsilon / 3+N \varepsilon / 3 N=\varepsilon . \square
\end{aligned}
$$

Proposition V.25. Assume Hypotheses (1)-(3), and (5). Let $i, j, k$, and l be distinct indices.

$$
K_{1}(z)=W_{i j}\left(1-P_{i j}\right)\left(z-H_{i j}\right)^{-1} V_{k l}\left(1-P_{i j, k l}\right)\left(z-H_{i j, k l}\right)^{-1} U_{i k}
$$

is compact and well behaved.

$$
K_{2}(z)=W_{i j}\left(1-P_{i j}\right)\left(z-H_{i j}\right)^{-1} V_{k l}\left(1-P_{i j, k l}\right)\left(z-H_{i j, k l}\right)^{-1}
$$

is compact and well behaved from $L_{\delta}^{2}\left(\mathbf{R}^{3 m}\right)$ to $L^{2}\left(\mathbf{R}^{3 m}\right)$, for any $\delta>1$.

Proof. Consider only $K_{1}(z)$; the proof for $K_{2}(z)$ is similar.

Compactness for large negative $\operatorname{Re} z$ is easily proved by expanding the resolvents as sums of graphs. It therefore suffices to prove $K_{1}(z)$ is well behaved. 
Using equation (V.6), we write $K_{1}(z)$ as a sum of four terms.

$$
\begin{aligned}
\mathrm{I}(z) & =W_{i j}\left(1-P_{i j}\right)\left(z-H_{i j}\right)^{-1} V_{k l}\left(1-P_{i j, k l}\right)\left(z-H_{i j}\right)^{-1} U_{i k}, \\
\mathrm{II}(z) & =W_{i j}\left(1-P_{i j}\right)\left(z-H_{i j}\right)^{-1} V_{k l}\left(1-P_{i j, k l}\right)\left(z-H_{k l}\right)^{-1} U_{i k}, \\
\mathrm{III}(z) & =-W_{i j}\left(1-P_{i j}\right)\left(z-H_{i j}\right)^{-1} V_{k l}\left(1-P_{i j, k l}\right)\left(z-H_{0}\right)^{-1} U_{i k}, \\
\mathrm{IV}(z) & =W_{i j}\left(1-P_{i j}\right)\left(z-H_{i j}\right)^{-1} V_{k l}\left(1-P_{i j, k l}\right) A(z)(1-M(z))^{-1} C(z) U_{i k} .
\end{aligned}
$$

Lemmas V.9 and V.12 control I(z), II(z), and III(z). Lemmas II.3 and V.21 show that $W_{i j}\left(1-P_{i j}\right)\left(z-H_{i j}\right)^{-1} V_{k l}\left(1-P_{i j, k l}\right) A(z)$ is well behaved, except that the boundary values are only strongly continuous. Lemma V.23 shows that $M(z)$ has similar properties.

By mimicking part of the proof of Proposition V.15, we see that the factor of $\left(1-P_{i j, k l}\right)$ in $\operatorname{IV}(z)$ removes the singularities of $(1-M(z))^{-1}$. Thus,

$$
W_{i j}\left(1-P_{i j}\right)\left(z-H_{i j}\right)^{-1} V_{k l}\left(1-P_{i j, k l}\right) A(z)(1-M(z))^{-1}
$$

is well behaved, except that the boundary values are strongly continuous. Lemma V.24 now shows that it suffices to prove $C(z) U_{i k}$ is well behaved and compact.

The first and third entries of $C(z) U_{i k}$ are compact for large negative $\operatorname{Re} z$, because they may be written as sums of connected graphs. The second and fourth entries are compact by the compactness argument used in the proof of Proposition V.16.

Lemmas V.9 and V.12 show that the first and third entries of $C(z) U_{i k}$ are well behaved. The second entry equals

$$
P_{i j} W_{k l}\left(1-P_{k l}\right)\left(z-H_{k l}\right)^{-1} U_{i k}+P_{i j} W_{k l} P_{k l}\left(z-H_{k l}\right)^{-1} U_{i k}
$$

Lemma V.6 controls the first term. The second may be written as

$$
\left(P_{i j}\left(1+x_{i j}^{2}\right)^{\alpha}\right)\left(W_{k l} P_{k l}\right)\left(\left(1+x_{i j}^{2}\right)^{-\alpha}\left(z-H_{k l}\right)^{-1} U_{i k}\right) \text {. }
$$

Apply Lemma II.3, Theorem II.9, and Corollary V.5. This shows that the second entry of $C(z) U_{i k}$ is well behaved. The fourth entry is handled in the same way.

Proposition V.26. Assume Hypotheses (1)-(3), and (5). Let $i, j, k$, and $l$ be distinct.

$$
K_{1}(z)=P_{i j} W_{k l}\left(1-P_{i j, k l}\right)\left(z-H_{i j, k l}\right)^{-1} U_{i k}
$$

is compact and well behaved.

$$
K_{2}(z)=P_{i j} W_{k l}\left(1-P_{i j, k l}\right)\left(z-H_{i j, k l}\right)^{-1}
$$

is compact and well behaved from $L_{\delta}^{2}\left(\mathbf{R}^{3 m}\right)$ to $L^{2}\left(\mathbf{R}^{3 m}\right)$, for any $\delta>1$.

Proof. Consider only $K_{1}(z)$; proof for $K_{2}(z)$ is similar.

$P_{i j}$ and $W_{k l}\left(1-P_{i j, k l}\right)$ commute. $P_{i j}\left(z-H_{i j, k l}\right)^{-1}=P_{i j}\left(z-E_{i j}-H_{k l}^{\prime}\right)^{-1}$, where $H_{k l}^{\prime}=H_{k l}-H_{0}^{(i j)}$. Moreover, $\left(1-P_{i j, k l}\right) P_{i j}=\left(1-P_{k l}\right) P_{i j}$. Therefore,

$$
K(z)=\left(P_{i j} W_{k l}\left(1-P_{k l}\right)\left(z-E_{i j}-H_{k l}^{\prime}\right)^{-1} \rho_{i j, k}\right)\left(\rho_{i j, k}^{-1} P_{i j} U_{i k}\right) \text {. }
$$


Lemma V. 4 bounds the second factor. $P_{i j}$ is (compact) $\otimes 1$;

$$
W_{k l}\left(1-P_{k l}\right)\left(z-E_{i j}-H_{k l}^{\prime}\right)^{-1} \rho_{i j, k}=1 \otimes X(z) .
$$

$X(z)$ is compact for large negative $\operatorname{Re} z$. The proof of this is the same as the compactness of the three body operator $W_{i j}\left(1-P_{i j}\right)\left(z-H_{i j}\right)^{-1} U_{i k}$ on $L^{2}\left(\mathbf{R}^{2 m}\right)$. $X(z)$ is well behaved by the proof of Lemma V.8 and the remark before Lemma V.10. Thus, $K_{1}(z)$ is compact and well behaved.

Proposition V.27. Assume Hypotheses (1)-(3), and (5). Let $i, j, k$, and $l$ be distinct, and let $n=j$ or $l$. The following are well behaved and compact:

$$
\begin{aligned}
K_{1}(z)= & W_{i j}\left(1-P_{i j}\right)\left(z-H_{i j}\right)^{-1} V_{k l}\left(1-P_{i j, k l}\right)\left(z-H_{i j, k l}\right)^{-1} \\
& \times V_{i k}\left(z-H_{i k}\right)^{-1} P_{i k} U_{j l}
\end{aligned}
$$

and

$$
\begin{aligned}
K_{2}(z)= & W_{i j}\left(1-P_{i j}\right)\left(z-H_{i j}\right)^{-1} V_{k l}\left(1-P_{i j, k l}\right)\left(z-H_{i j, k l}\right)^{-1} \\
& \times V_{i k}\left(z-H_{i k}\right)^{-1} P_{i k} \rho_{i k, n} .
\end{aligned}
$$

Proof. Consider only $K_{1}(z)$; the proof for $K_{2}(z)$ is similar.

For large negative $\operatorname{Re} z, W_{i k}\left(z-H_{i k}\right)^{-1} P_{i k} U_{j l}$ is bounded, so compactness follows from Proposition V.25. Hence, it suffices to prove $K_{1}(z)$ is well behaved.

Using equation (V.6), we write $K_{1}(z)$ as a sum of four terms:

$$
\begin{aligned}
\mathrm{I}(z)= & -W_{i j}\left(1-P_{i j}\right)\left(z-H_{i j}\right)^{-1} V_{k l}\left(1-P_{i j, k l}\right)\left(z-H_{0}\right)^{-1} \\
& \times V_{i k}\left(z-H_{i k}\right)^{-1} P_{i k} U_{j l}, \\
\mathrm{II}(z)= & W_{i j}\left(1-P_{i j}\right)\left(z-H_{i j}\right)^{-1} V_{k l}\left(1-P_{i j, k l}\right)\left(z-H_{i j}\right)^{-1} \\
& \times V_{i k}\left(z-H_{i k}\right)^{-1} P_{i k} U_{j l}, \\
\mathrm{III}(z)= & W_{i j}\left(1-P_{i j}\right)\left(z-H_{i j}\right)^{-1} V_{k l}\left(1-P_{i j, k l}\right)\left(z-H_{k l}\right)^{-1} \\
& \times V_{i k}\left(z-H_{i k}\right)^{-1} P_{i k} U_{j l}, \\
\mathrm{IV}(z)= & W_{i j}\left(1-P_{i j}\right)\left(z-H_{i j}\right)^{-1} V_{k l}\left(1-P_{i j, k l}\right) A(z)(1-M(z))^{-1} \\
& \times C(z) V_{i k}\left(z-H_{i k}\right)^{-1} P_{i k} U_{j l} \cdot \\
\mathrm{I}(z)=W_{i j}(1- & \left.P_{i j}\right)\left(z-H_{i j}\right)^{-1} V_{k l}\left(1-P_{i j, k l}\right)\left[\left(z-H_{i k}\right)^{-1}-\left(z-H_{0}\right)^{-1}\right] P_{i k} U_{j l} \cdot
\end{aligned}
$$

This is well behaved by Lemma V.12.

$$
\begin{aligned}
\mathrm{II}(z)= & -\mathrm{I}(z)+W_{i j}\left(1-P_{i j}\right)\left(z-H_{i j}\right)^{-1} V_{k l}\left(1-P_{i j, k l}\right)\left(z-H_{i j}\right)^{-1} \\
& \times V_{i j}\left[\left(z-H_{i k}\right)^{-1}-\left(z-H_{0}\right)^{-1}\right] P_{i k} U_{j l} \\
= & -\mathrm{I}(z)+\left(W_{i j}\left(1-P_{i j}\right)\left(z-H_{i j}\right)^{-1} V_{k l}\left(1-P_{i j, k l}\right)\left(1-P_{i j}\right)\left(z-H_{i j}\right)^{-1} U_{i j}\right) \\
& \times\left(W_{i j}\left[\left(z-H_{i k}\right)^{-1}-\left(z-H_{0}\right)^{-1}\right] P_{i k} U_{j l}\right) .
\end{aligned}
$$


Apply Lemmas V.9, V.10, and II.3:

$$
\begin{aligned}
\mathrm{III}(z)= & -\mathrm{I}(z)+W_{i j}\left(1-P_{i j}\right)\left(z-H_{i j}\right)^{-1} V_{k l}\left(1-P_{i j, k l}\right)\left(z-H_{k l}\right)^{-1} \\
& \times V_{k l}\left[\left(z-H_{i k}\right)^{-1}-\left(z-H_{0}\right)^{-1}\right] P_{i k} U_{j l} \\
= & -\mathrm{I}(z)+\left(W_{i j}\left(1-P_{i j}\right)\left(z-H_{i j}\right)^{-1} V_{k l}\left(1-P_{i j, k l}\right)\left(z-H_{k l}\right)^{-1}\right. \\
& \left.\times U_{k l}\left(1+x_{i k}^{2}\right)^{-\alpha}\right)\left(\left(1+x_{i k}^{2}\right)^{\alpha} W_{k l} P_{i k}\left(z-H_{i k}\right)^{-1} U_{j l}\right) \\
& -W_{i j}\left(1-P_{i j}\right)\left(z-H_{i j}\right)^{-1} V_{k l}\left(1-P_{i j, k l}\right) \\
& \times\left[\left(z-H_{k l}\right)^{-1}-\left(z-H_{0}\right)^{-1}\right] P_{i k} U_{j l} .
\end{aligned}
$$

Lemmas V.9 and V.12 control the last term. Lemma V.10 and the fall off of $\Psi_{i k}$ control the second factor of the middle term. If $U_{k l}$ is bounded, then Lemmas V.4, V.9, and V.12 control the first factor. When $U_{k l}$ is unbounded, a density argument shows that it suffices to prove uniform boundedness of the first factor. To do this, we rewrite this first factor as

$$
\begin{aligned}
& W_{i j}\left(1-P_{i j}\right)\left(z-H_{i j}\right)^{-1} V_{k l}\left(1-P_{i j, k l}\right)\left(z-H_{k l}\right)^{-1} \\
& \quad \times P_{k l} U_{k l}\left(1+x_{i k}^{2}\right)^{-\alpha}+W_{i j}\left(1-P_{i j}\right)\left(z-H_{i j}\right)^{-1} \\
& \quad \times V_{k l}\left(1-P_{i j, k l}\right)\left(z-H_{k l}\right)^{-1}\left(1-P_{k l}\right) U_{k l}\left(1+x_{i k}^{2}\right)^{-\alpha}
\end{aligned}
$$

Lemmas V.8 and V.21 show the second term is uniformly bounded. Corollary V.5 and Lemmas V.4 and V.12 control the first term.

To control IV $(z)$, we proceed as in the proof of Proposition V.25. This shows that we need only prove $C(z) V_{i k}\left(z-H_{i k}\right)^{-1} P_{i k} U_{j l}$ is compact and well behaved. Since $W_{i k}\left(z-H_{i k}\right)^{-1} P_{i k} U_{j l}$ is bounded for large negative $\operatorname{Re} z$, compactness follows from arguments in the proof of Proposition V.25. To prove $C(z) V_{i k}\left(z-H_{i k}\right)^{-1} P_{i k} U_{j l}$ is well behaved, notice that the first and third entries are of the same form as $\operatorname{III}(z)$, above. The second entry may be rewritten as

$$
\begin{aligned}
P_{i j} W_{k l} & {\left[\left(z-H_{i k}\right)^{-1}-\left(z-H_{0}\right)^{-1}\right] P_{i k} U_{j l} } \\
& +P_{i j} W_{k l}\left(z-H_{k l}\right)^{-1} V_{k l}\left(z-H_{i k}\right)^{-1} P_{i k} U_{j l} \\
& -P_{i j} W_{k l}\left[\left(z-H_{k l}\right)^{-1}-\left(z-H_{0}\right)^{-1}\right] P_{i k} U_{j l}
\end{aligned}
$$

Replace each $\left(z-H_{k l}\right)^{-i}$ in these expressions by $\left(z-H_{k l}\right)^{-1} P_{k l}+\left(z-H_{k l}\right)^{-1}(1$ $\left.-P_{k l}\right)$. The only terms which are not controlled by various lemmas are those containing the factor $P_{k l}$. To control them, notice that $W_{k l} P_{k l}$ and $P_{k l} U_{k l}$ are bounded. Then use Lemma V.4 and the fall off of $\Psi_{i j}$ and $\Psi_{i k}$ to obtain factors which are controlled by Lemma II.3. This controls the second entry of $C(z) V_{i k}(z-$ $\left.H_{i k}\right)^{-1} P_{i k} U_{j l}$. The same method applies to the fourth entry.

Proposition V.28. Assume Hypotheses (1)-(3), and (5). Let $i, j, k$, and $l$ be distinct, and let $n=i$ or $j$. The following are well behaved and compact:

$$
K_{1}(z)=P_{i j} W_{k l}\left(1-P_{i j, k l}\right)\left(z-H_{i j, k l}\right)^{-1} V_{i k}\left(z-H_{i k}\right)^{-1} P_{i k} U_{j l}
$$


and

$$
K_{2}(z)=P_{i j} W_{k l}\left(1-P_{i j, k l}\right)\left(z-H_{i j, k l}\right)^{-1} V_{i k}\left(z-H_{i k}\right)^{-1} P_{i k} \rho_{i k, n} .
$$

Proof. Consider only $K_{1}(z)$; the proof for $K_{2}(z)$ is similar.

For large negative $\operatorname{Re} z, W_{i k}\left(z-H_{i k}\right)^{-1} P_{i k} U_{j l}$ is bounded, so compactness follows from Proposition V.26. It is therefore sufficient to show $K_{1}(z)$ is well behaved.

$P_{i j}$ commutes with $W_{k l}$, and $P_{i j}\left(1-P_{i j, k l}\right)=P_{i j}\left(1-P_{k l}\right)$. So,

$$
\begin{aligned}
K_{1}(z)= & P_{i j} W_{k l}\left(1-P_{k l}\right)\left(z-H_{k l}\right)^{-1} V_{i k}\left(z-H_{i k}\right)^{-1} P_{i k} U_{j l} \\
& +P_{i j} W_{k l}\left(1-P_{k l}\right)\left(z-H_{k l}\right)^{-1} V_{i j}\left(1-P_{i j, k l}\right)\left(z-H_{i j, k l}\right)^{-1} \\
& \times V_{i k}\left(z-H_{i k}\right)^{-1} P_{i k} U_{j l} .
\end{aligned}
$$

Lemma V.12 controls the first term. Proposition V.27 controls the second.

Proposition V.29. Assume Hypotheses (1)-(3), and (5). Let $i, j, k$, and $l$ be distinct; let $n=i, j$, or $k$; let $p$ be any index not equal to $n$ or $l$; let $q$ be the index not equal $n, l$, or $p$. The following are well behaved and compact:

$$
\begin{aligned}
K_{1}(z)= & \rho_{i j, k}^{-1} P_{i j}\left(V_{i k}+V_{j k}\right)\left(z-H_{i j k}\right)^{-1}\left(1-P_{i j k}\right) \\
& \times\left[V_{n l}\left(z-H_{n l}\right)^{-1}\left(V_{n p}+V_{l p}\right)+V_{l p}\left(z-H_{l p}\right)^{-1}\left(V_{n p}+V_{n l}\right)\right] \\
& \times\left(z-H_{n l p}\right)^{-1} P_{n l p} \rho_{n l p}
\end{aligned}
$$

and

$$
\begin{aligned}
K_{2}(z)= & \rho_{i j, k}^{-1} P_{i j}\left(V_{i k}+V_{j k}\right)\left(z-H_{i j k}\right)^{-1}\left(1-P_{i j k}\right) \\
& \times V_{n l}\left(z-H_{n l}\right)^{-1} V_{p q}\left(z-H_{n l, p q}\right)^{-1} P_{n l, p q} \rho_{n l, p q} .
\end{aligned}
$$

Proof. Consider only $K_{1}(z)$; proof for $K_{2}(z)$ is similar.

For large negative $\operatorname{Re} z$,

$$
W_{n l}\left(z-H_{n l}\right)^{-1}\left(V_{n p}+V_{p l}\right)\left(z-H_{n p}\right)^{-1} P_{n l p} \rho_{n p}
$$

and

$$
W_{l p}\left(z-H_{l p}\right)^{-1}\left(V_{n p}+V_{n l}\right)\left(z-H_{n l p}\right)^{-1} P_{n l p} \rho_{n l p}
$$

are uniformly bounded, so compactness and $\lim _{\operatorname{Re} z \rightarrow-\infty}\left\|K_{1}(z)\right\|=0$ follow from Proposition V.16.

$$
\begin{aligned}
& \text { Let } X(z)=\rho_{i j, k}^{-1} P_{i j}\left(V_{i k}+V_{j k}\right)\left(z-H_{i j k}\right)^{-1}\left(1-P_{i j k}\right) \text {. } \\
& K_{1}(z)=X(z)\left(V_{n l}+V_{p l}\right)\left(z-H_{n l p}\right)^{-1} P_{n l p} \rho_{n l p} \\
& -X(z) V_{n l}\left(z-H_{n l}\right)^{-1} P_{n l p} \rho_{n l p}-X(z) V_{p l}\left(z-H_{p l}\right)^{-1} P_{n l p} \rho_{n l p} \text {. }
\end{aligned}
$$

The second and third terms can each be broken into two terms which are controlled by Proposition V.18 or Lemma V.8 and Proposition V.16. 
Since $\{i, j, k\}=\{n, p, q\}$, the first term equals:

$$
\begin{aligned}
\rho_{i j, k}^{-1} P_{i j} & \left(V_{i k}+V_{j k}\right)\left(1-P_{i j k}\right)\left(z-H_{n p}\right)^{-1}\left(V_{n l}+V_{p l}\right)\left(z-H_{n l p}\right)^{-1} \\
& \times P_{n l p} \rho_{n l p}+\rho_{i j, k}^{-1} P_{i j}\left(V_{i k}+V_{j k}\right)\left(1-P_{i j k}\right)\left(z-H_{i j k}\right)^{-1} \\
& \times\left(V_{n q}+V_{p q}\right)\left(z-H_{n p}\right)^{-1}\left(V_{n l}+V_{p l}\right)\left(z-H_{n p}\right)^{-1} P_{n l p} \rho_{n l p} .
\end{aligned}
$$

This may be rewritten as a sum of four terms:

$$
\begin{aligned}
\mathrm{I}(z)= & \rho_{i j, k}^{-1} P_{i j}\left(V_{i k}+V_{j k}\right)\left(1-P_{i j k}\right)\left(z-H_{n l p}\right)^{-1} P_{n l p} \rho_{n l p}, \\
\mathrm{II}(z)= & -\rho_{i j, k}^{-1} P_{i j}\left(V_{i k}+V_{j k}\right)\left(1-P_{i j k}\right)\left(z-H_{n p}\right)^{-1} P_{n l p} \rho_{n l p}, \\
\mathrm{III}(z)= & \rho_{i j, k}^{-1} P_{i j}\left(V_{i k}+V_{j k}\right)\left(1-P_{i j k}\right) \\
& \times\left(z-H_{i j k}\right)^{-1}\left(V_{n q}+V_{p q}\right)\left(z-H_{n l p}\right)^{-1} P_{n l p} \rho_{n l p}, \\
\mathrm{IV}(z)= & -\rho_{i j, k} P_{i j}\left(V_{i k}+V_{j k}\right)\left(1-P_{i j k}\right) \\
& \times\left(z-H_{i j k}\right)^{-1}\left(V_{n q}+V_{p q}\right)\left(z-H_{n p}\right)^{-1} P_{n l p} \rho_{n l p} . \\
\mathrm{I}(z)=\rho_{i j, k}^{-1} P_{i j}\left(V_{i k}+\right. & \left.V_{j k}\right)\left(z-H_{n l p}\right)^{-1} P_{n l p} \rho_{n l p} \\
- & \rho_{i j, k}^{-1} P_{i j}\left(V_{i k}+V_{j k}\right) P_{i j k}\left(z-H_{n l p}\right)^{-1} P_{n l p} \rho_{n l p} .
\end{aligned}
$$

The first term in this expression equals

$$
\begin{aligned}
& {\left[\rho_{i j, k}^{-1} P_{i j} U_{i k}\left(1+x_{i j}^{2}\right)^{\alpha}\right]\left[\left(1+x_{i j}^{2}\right)^{-\alpha} W_{i k}\left(z-K_{n l p}-E_{n p}\right)^{-1} P_{n l p} \rho_{n l p}\right]} \\
& \quad+\left[\rho_{i j, k}^{-1} P_{i j} U_{j k}\left(1+x_{i j}^{2}\right)^{\alpha}\right]\left[\left(1+x_{i j}^{2}\right)^{-\alpha} W_{j k}\left(z-K_{n l p}-E_{n l p}\right)^{-1} P_{n l p} \rho_{n l p}\right]
\end{aligned}
$$

Lemma V.4 bounds the first factors. The methods of Lemma II.3 control the second factors. The second term in the last expression for $\mathrm{I}(z)$ equals

$$
\begin{aligned}
& -\left(\rho_{i j, k}^{-1} P_{i j} U_{i k}\right)\left(W_{i k} P_{i j k}\right)\left(P_{i j k} P_{n l p} \rho_{n l p}^{-1}\right)\left(\rho_{n l p}\left(z-H_{n l p}\right)^{-1} \rho_{n l p}\right) \\
& -\left(\rho_{i j, k}^{-1} P_{i j} U_{j k}\right)\left(W_{j k} P_{i j k}\right)\left(P_{i j k} P_{n l p} \rho_{n l p}^{-1}\right)\left(\rho_{n l p}\left(z-H_{n l p}\right)^{-1} \rho_{n l p}\right) .
\end{aligned}
$$

Lemma V.4 bounds the first factors. Corollary V.5 bounds the second factors. The proof of Lemma V.4 bounds the third factors. Lemma II.3 controls the final factors.

$$
\begin{aligned}
\operatorname{III}(z)= & \rho_{i j, k}^{-1} P_{i j}\left(V_{i k}+V_{j k}\right)\left(1-P_{i j k}\right)\left(z-H_{i j k}\right)^{-1} V_{n q}\left(z-H_{n l p}\right)^{-1} P_{n l p} \rho_{n l p} \\
& +\rho_{i j, k}^{-1} P_{i j}\left(V_{i k}+V_{j k}\right)\left(1-P_{i j k}\right)\left(z-H_{i j k}\right)^{-1} V_{p q}\left(z-H_{n q p}\right)^{-1} P_{n l p} \rho_{n l p} .
\end{aligned}
$$

Since $\left(z-H_{n l p}\right)^{-1} P_{n l p}=\left(z-K_{n l p}-E_{n l p}\right)^{-1} P_{n l p}$, Lemma V.4 and Lemma II.3 show $\left(1+x_{p l}^{2}+x_{n l}^{2}+x_{n p}^{2}\right)^{\alpha} W_{n q}\left(z-H_{n l p}\right)^{-1} P_{n l p} \rho_{n l p}$ is well behaved. Following the proof of Proposition V.16,

$$
\rho_{i j, k}^{-1} P_{i j}\left(V_{i k}+V_{j k}\right)\left(1-P_{i j k}\right)\left(z-H_{i j k}\right)^{-1} U_{n q}\left(1+x_{n p}^{2}+x_{n l}^{2}+x_{p l}^{2}\right)^{-\alpha}
$$

is well behaved. This controls the first term in the last expression for $\operatorname{III}(z)$. The same proof controls the second term.

Since $\{n, p, q\}=\{i, j, k\}$,

$$
\mathrm{II}(z)+\mathrm{IV}(z)=-\rho_{i j, k}^{-1} P_{i j}\left(V_{i k}+V_{j k}\right)\left(1-P_{i j k}\right)\left(z-H_{i j k}\right)^{-1} P_{n p} \rho_{n p} .
$$

The proof of Proposition V.16 shows this is well behaved. 
Proposition V.30. Assume Hypotheses (1)-(3), and (5). Let $i, j, k$, and $l$ be distinct. The following are compact and well behaved:

$$
\begin{aligned}
K_{1}(z)= & P_{i j} W_{k l}\left(1-P_{i j, k l}\right)\left(z-H_{i j, k l}\right)^{-1} \\
& \times\left[V_{i k}\left(z-H_{i k}\right)^{-1} V_{j l}+V_{j l}\left(z-H_{j l}\right)^{-1} V_{i k}\right]\left(z-H_{i k, j l}\right)^{-1} P_{i j, k l} \rho_{i j, k l}, \\
K_{2}(z)= & P_{i j} W_{k l}\left(1-P_{i j, k l}\right)\left(z-H_{i j, k l}\right)^{-1} \\
& \times\left[V_{i k}\left(z-H_{i k}\right)^{-1}\left(V_{j k}+V_{i j}\right)+V_{j k}\left(z-H_{j k}\right)^{-1}\left(V_{i k}+V_{i j}\right)\right] \\
& \times\left(z-H_{i j k}\right)^{-1} P_{i j k} \rho_{i j k}, \\
K_{3}(z)= & P_{i j} W_{k l}\left(1-P_{i j, k l}\right)\left(z-H_{i j, k l}\right)^{-1} \\
& \times\left[V_{i l}\left(z-H_{i l}\right)^{-1}\left(V_{i k}+V_{l k}\right)+V_{i k}\left(z-H_{i k}\right)^{-1}\left(V_{i l}+V_{l k}\right)\right] \\
& \times\left(z-H_{i l k}\right)^{-1} P_{i l k} \rho_{i l k} .
\end{aligned}
$$

Proof. Compactness and the limiting behavior as $\operatorname{Re} z \rightarrow-\infty$ follow from Proposition V.26 and simple bounds.

Let

$$
\begin{gathered}
X(z)=P_{i j} W_{k l}\left(z-H_{i j, k l}\right)^{-1}\left(1-P_{i j, k l}\right) \\
=W_{k l}\left(z-H_{i j, k l}\right)^{-1} P_{i j}\left(1-P_{k l}\right) \\
K_{1}(z)=X(z)\left(V_{i k}+V_{j l}\right)\left(z-H_{i k, j l}\right)^{-1} P_{i k, j l} \rho_{i k, j l} \\
-X(z) V_{i k}\left(z-H_{i k}\right)^{-1} P_{i k, j l} \rho_{i k, j l}-X(z) V_{j l}\left(z-H_{j l}\right)^{-1} P_{i k, j l} \rho_{i k, j l} .
\end{gathered}
$$

Trivial extensions of Proposition V.28 control the last two terms. The first term equals:

$$
\begin{aligned}
& {\left[P_{i j} W_{k l}\left(z-H_{i j, k l}\right)^{-1}\left(1-P_{i j, k l}\right) U_{i k}\left(1+x_{i j}^{2}\right)^{\alpha}\right]} \\
& \times\left[\left(1+x_{i j}^{2}\right)^{-\alpha} W_{i k}\left(z-H_{i k, j l}\right)^{-1} P_{i k, j l} \rho_{i k, j l}\right] \\
& +\left[P_{i j} W_{k l}\left(z-H_{i j, k l}\right)^{-1}\left(1-P_{i j, k l}\right) U_{j l}\left(1+x_{i j}^{2}\right)^{\alpha}\right] \\
& \quad \times\left[\left(1+x_{i j}^{2}\right)^{-\alpha} W_{j l}\left(z-H_{i k, j l}\right)^{-1} P_{i k, j l} \rho_{i k, j l}\right] .
\end{aligned}
$$

Due to the fall off of $\Psi_{i j}$, the proof of Proposition V.26 can easily be extended to show the first factors are well behaved. The methods of Lemmas II.3 and V.4 show the second factors are well behaved.

$$
\begin{aligned}
K_{2}(z)= & X(z)\left(V_{i k}+V_{j k}\right)\left(z-H_{i j k}\right)^{-1} P_{i j k} \rho_{i j k} \\
& -X(z) V_{i k}\left(z-H_{i k}\right)^{-1} P_{i j k} \rho_{i j k}-X(z) V_{j k}\left(z-H_{j k}\right)^{-1} P_{i j k} \rho_{i j k} .
\end{aligned}
$$

The last two terms can each be written as sums of two terms, which are well behaved by Proposition V.28 or Proposition V.26 and Lemma V.8. The first term 
equals:

$$
\begin{aligned}
P_{i j} W_{k l}\left(1-P_{i j, k l}\right)\left(z-H_{i j}\right)^{-1}\left(V_{i k}+V_{j k}\right)\left(z-H_{i j k}\right)^{-1} P_{i j k} \rho_{i j k} \\
\quad+P_{i j} W_{k l}\left(1-P_{i j, k l}\right)\left(z-H_{i j, k l}\right)^{-1} V_{k l}\left(z-H_{i j}\right)^{-1}\left(V_{i k}+V_{j k}\right) \\
\quad \times\left(z-H_{i j k}\right)^{-1} P_{i j k} \rho_{i j k} .
\end{aligned}
$$

We write this as a sum of four terms:

$$
\begin{gathered}
\mathrm{I}(z)=P_{i j} W_{k l}\left(1-P_{i j, k l}\right)\left(z-H_{i j k}\right)^{-1} P_{i j k} \rho_{i j k}, \\
\mathrm{II}(z)=-P_{i j} W_{k l}\left(1-P_{i j, k l}\right)\left(z-H_{i j}\right)^{-1} P_{i j k} \rho_{i j k}, \\
\mathrm{III}(z)=P_{i j} W_{k l}\left(1-P_{i j, k l}\right)\left(z-H_{i j, k l}\right)^{-1} V_{k l}\left(z-H_{i j k}\right)^{-1} P_{i j k} \rho_{i j k}, \\
\mathrm{IV}(z)=-P_{i j} W_{k l}\left(1-P_{i j, k l}\right)\left(z-H_{i j, k l}\right)^{-1} V_{k l}\left(z-H_{i j}\right)^{-1} P_{i j k} \rho_{i j k} . \\
\mathrm{I}(z)=\left(P_{i j} W_{k l}\left(1-P_{i j, k l}\right) P_{i j k} \rho_{i j k}^{-1}\right)\left(\rho_{i j k}\left(z-H_{i j k}\right)^{-1} \rho_{i j k}\right) .
\end{gathered}
$$

Apply Lemmas V.4 and II.3 to control this.

$$
\begin{aligned}
\operatorname{III}(z)= & \left(P_{i j} W_{k l}\left(1-P_{i j, k l}\right)\left(z-H_{i j, k l}\right)^{-1} U_{k l}\right)\left(W_{k l} P_{i j k} \rho_{i j k}^{-1}\right) \\
& \times\left(\rho_{i j k}\left(z-H_{i j k}\right)^{-1} \rho_{i j k}\right) .
\end{aligned}
$$

The proof of Proposition V.26 easily generalizes to control the first factor; Lemma V.4 bounds the second factor; and Lemma II.3 controls the third.

$$
\mathrm{II}(z)+\operatorname{IV}(z)=-P_{i j} W_{k l}\left(1-P_{i j, k l}\right)\left(z-H_{i j, k l}\right)^{-1} P_{i j k} \rho_{i j k} \cdot
$$

The proof of Proposition V.26 generalizes to control this term:

$$
\begin{aligned}
K_{3}(z)= & X(z)\left(V_{i l}+V_{i k}\right)\left(z-H_{i l k}\right)^{-1} P_{i l k} \rho_{i l k} \\
& -X(z) V_{i l}\left(z-H_{i l}\right)^{-1} P_{i k l} \rho_{i l k}-X(z) V_{i k}\left(z-H_{i k}\right)^{-1} P_{i l k} \rho_{i l k} .
\end{aligned}
$$

The second and third terms are of a form studied in the proof for $K_{2}(z)$. The first term equals

$$
\begin{aligned}
{\left[P_{i j} W_{k l}\right.} & \left.\left(1-P_{i j, k l}\right)\left(z-H_{i j, k l}\right)^{-1} U_{i l}\left(1+x_{i j}^{2}\right)^{\alpha}\right] \\
& \times\left[\left(1+x_{i j}^{2}\right)^{-\alpha} W_{i l}\left(z-H_{i l k}\right)^{-1} P_{i l k} \rho_{i l k}\right] \\
& +\left[P_{i j} W_{k l}\left(1-P_{i j, k l}\right)\left(z-H_{i j, k l}\right)^{-1} U_{i k}\left(1+x_{i j}^{2}\right)^{\alpha}\right] \\
& \times\left[\left(1+x_{i j}^{2}\right)^{-\alpha} W_{i k}\left(z-H_{i l k}\right)^{-1} P_{i l k} \rho_{i l k}\right]
\end{aligned}
$$

The first factors are of the same form as terms which have been controlled in the proof for $K_{1}(z)$. The second factors are controlled by methods of Lemmas V.4 and II.3. 
Proposition V.31. Assume Hypotheses (1)-(3), and (5). Let $i, j, k$ and $l$ be distinct. The following are well behaved and compact:

$$
\begin{aligned}
K_{1}(z)= & W_{i j}\left(1-P_{i j}\right)\left(z-H_{i j}\right)^{-1}\left(V_{i k}+V_{j k}\right)\left(1-P_{i j k}\right)\left(z-H_{i j k}\right)^{-1} \\
& \times\left[V_{i l}\left(z-H_{i l}\right)^{-1}\left(V_{j l}+V_{i j}\right)+V_{j l}\left(z-H_{j l}\right)^{-1}\left(V_{i l}+V_{i j}\right)\right] \\
& \times\left(z-H_{i j l}\right)^{-1} P_{i j l} \rho_{i j l}, \\
K_{2}(z)= & W_{i j}\left(1-P_{i j}\right)\left(z-H_{i j}\right)^{-1}\left(V_{i k}+V_{j k}\right)\left(1-P_{i j k}\right)\left(z-H_{i j k}\right)^{-1} \\
& \times V_{k l}\left(z-H_{k l}\right)^{-1} V_{i j}\left(z-H_{i j, k l}\right)^{-1} P_{i j, k l} \rho_{i j, k l}, \\
K_{3}(z)= & W_{i j}\left(1-P_{i j}\right)\left(z-H_{i j}\right)^{-1} V_{k l}\left(1-P_{i j, k l}\right)\left(z-H_{i j, k l}\right)^{-1} \\
& \times\left[V_{i k}\left(z-H_{i k}\right)^{-1}\left(V_{j k}+V_{i j}\right)+V_{j k}\left(z-H_{j k}\right)^{-1}\left(V_{i k}+V_{i j}\right)\right] \\
& \times\left(z-H_{i j k}\right)^{-1} P_{i j k} \rho_{i j k}, \\
K_{4}(z)= & W_{i j}\left(1-P_{i j}\right)\left(z-H_{i j}\right)^{-1} V_{k l}\left(1-P_{i j, k l}\right)\left(z-H_{i j, k l}\right)^{-1} \\
& \times\left[V_{i k}\left(z-H_{i k}\right)^{-1} V_{j l}+V_{j l}\left(z-H_{j l}\right)^{-1} V_{i k}\right]\left(z-H_{i k, j l}\right)^{-1} P_{i j, k l} \rho_{i j, k l} .
\end{aligned}
$$

Proof. Consider only $K_{1}(z)$; the proofs for the others are similar.

Since

$$
W_{i l}\left(z-H_{i l}\right)^{-1}\left(V_{j l}+V_{i j}\right)\left(z-H_{i j l}\right)^{-1} P_{i j l} \rho_{i j l}
$$

and

$$
W_{j l}\left(z-H_{j l}\right)^{-1}\left(V_{i l}+V_{i j}\right)\left(z-H_{i j l}\right)^{-1} P_{i j l} \rho_{i j l}
$$

are bounded for large negative $\operatorname{Re} z$, compactness of $K_{1}(z)$ at large negative $\operatorname{Re} z$ follows from Proposition V.15.

Let

$$
X(z)=W_{i j}\left(1-P_{i j}\right)\left(z-H_{i j}\right)^{-1}\left(V_{i k}+V_{j k}\right)\left(1-P_{i j k}\right)\left(z-H_{i j k}\right)^{-1} .
$$

Then,

$$
\begin{aligned}
K_{1}(z)= & X(z)\left(V_{i l}+V_{j l}\right)\left(z-H_{i j l}\right)^{-1} P_{i j l} \rho_{i j l} \\
& -X(z) V_{i l}\left(z-H_{i l}\right)^{-1} P_{i j l} \rho_{i j l}-X(z) V_{j l}\left(z-H_{j l}\right)^{-1} P_{i j l} \rho_{i j l} \cdot
\end{aligned}
$$

The second and third terms can each be broken into two terms which are well behaved by Proposition V.17 or Lemma V.8 and Proposition V.15. The first term equals

$$
\begin{aligned}
W_{i j}(1 & \left.-P_{i j}\right)\left(z-H_{i j}\right)^{-1}\left(V_{i k}+V_{j k}\right)\left(1-P_{i j k}\right)\left(z-H_{i j}\right)^{-1}\left(V_{i l}+V_{j l}\right) \\
& \times\left(z-H_{i j l}\right)^{-1} P_{i j l} \rho_{i j l} \\
& +W_{i j}\left(1-P_{i j}\right)\left(z-H_{i j}\right)^{-1}\left(V_{i k}+V_{j k}\right)\left(1-P_{i j k}\right)\left(z-H_{i j k}\right)^{-1}\left(V_{i k}+V_{j k}\right) \\
& \times\left(z-H_{i j}\right)^{-1}\left(V_{i l}+V_{j l}\right)\left(z-H_{i j l}\right)^{-1} P_{i j l} \rho_{i j l} .
\end{aligned}
$$


This may be written as a sum of four terms:

$$
\begin{aligned}
\mathrm{I}(z)= & W_{i j}\left(1-P_{i j}\right)\left(z-H_{i j}\right)\left(V_{i k}+V_{j k}\right)\left(1-P_{i j k}\right)\left(z-H_{i j l}\right)^{-1} P_{i j l} \rho_{i j l}, \\
\mathrm{II}(z)= & -W_{i j}\left(1-P_{i j}\right)\left(z-H_{i j}\right)^{-1}\left(V_{i k}+V_{j k}\right)\left(1-P_{i j k}\right)\left(z-H_{i j}\right)^{-1} P_{i j l} \rho_{i j l}, \\
\mathrm{III}(z)= & W_{i j}\left(1-P_{i j}\right)\left(z-H_{i j}\right)^{-1}\left(V_{i k}+V_{j k}\right)\left(1-P_{i j k}\right)\left(z-H_{i j k}\right)^{-1} \\
& \times\left(V_{i k}+V_{j k}\right)\left(z-H_{i j l}\right)^{-1} P_{i j l} \rho_{i j l}, \\
\mathrm{IV}(z)= & -W_{i j}\left(1-P_{i j}\right)\left(z-H_{i j}\right)^{-1}\left(V_{i k}+V_{j k}\right)\left(1-P_{i j k}\right)\left(z-H_{i j k}\right)^{-1} \\
& \times\left(V_{i k}+V_{j k}\right)\left(z-H_{i j}\right)^{-1} P_{i j l} \rho_{i j l} \cdot \\
\mathrm{I}(z)= & \left(W_{i j}\left(1-P_{i j}\right)\left(z-H_{i j}\right)^{-1} U_{i k}\right)\left(W_{i k} P_{i j l} \rho_{i j l}^{-1}\right)\left(\rho_{i j l}\left(z-H_{i j l}\right)^{-1} \rho_{i j l}\right) \\
+ & \left(W_{i j}\left(1-P_{i j}\right)\left(z-H_{i j}\right)^{-1} U_{j k}\right)\left(W_{j k} P_{i j l} \rho_{i j l}^{-1}\right)\left(\rho_{i j l}\left(z-H_{i j l}\right)^{-1} \rho_{i j l}\right) \\
& -\left(W_{i j}\left(1-P_{i j}\right)\left(z-H_{i j}\right)^{-1} U_{i k}\right)\left(W_{i k} P_{i j k} P_{i j l} \rho_{i j l}^{-1}\right)\left(\rho_{i j l}\left(z-H_{i j l}\right)^{-1} \rho_{i j l}\right) \\
& -\left(W_{i j}\left(1-P_{i j}\right)\left(z-H_{i j}\right)^{-1} U_{j k}\right)\left(W_{j k} P_{i j k} P_{i j l} \rho_{i j l}^{-1}\right)\left(\rho_{i j l}\left(z-H_{i j l}\right)^{-1} \rho_{i j l}\right) .
\end{aligned}
$$

Apply Lemmas V.4, V.8, and II.3.

$\operatorname{III}(z)$ and II $(z)+\operatorname{IV}(z)$ are well behaved for the same reasons that $\operatorname{III}(z)$ and $\mathrm{II}(z)+\operatorname{IV}(z)$ in Proposition V.29 are well behaved. The only difference is that Proposition V.15 must be used in place of Proposition V.16.

Proposition V.32. Assume Hypotheses (1)-(3), and (5). Let $i, j, k$, and $l$ be distinct.

$$
\begin{aligned}
K_{1}(z)= & W_{i j}\left(1-P_{i j}\right)\left(z-H_{i j}\right)^{-1}\left(V_{i k}+V_{j k}\right)\left(1-P_{i j k}\right)\left(z-H_{i j k}\right)^{-1} \\
& \times V_{i l}\left(z-H_{i l}\right)^{-1} V_{j k}\left(z-H_{i l j k}\right)^{-1} P_{i l j k} \rho_{i l, j k}
\end{aligned}
$$

is well behaved and compact.

If Hypothesis (4) also holds, then the following are well behaved and compact:

$$
\begin{aligned}
K_{2}(z)= & W_{i j}\left(1-P_{i j}\right)\left(z-H_{i j}\right)^{-1}\left(V_{i k}+V_{j k}\right)\left(1-P_{i j k}\right)\left(z-H_{i j k}\right)^{-1} \\
& \times\left[V_{i l}\left(z-H_{i l}\right)^{-1}\left(V_{i k}+V_{l k}\right)+V_{k l}\left(z-H_{k l}\right)^{-1}\left(V_{i k}+V_{i l}\right)\right] \\
& \times\left(z-H_{i l k}\right)^{-1} P_{i l k} \rho_{i l k} \cdot \\
K_{3}(z)= & W_{i j}\left(1-P_{i j}\right)\left(z-H_{i j}\right)^{-1} V_{k l}\left(1-P_{i j, k l}\right)\left(z-H_{i j, k l}\right)^{-1} \\
& \times\left[V_{i l}\left(z-H_{i l}\right)^{-1}\left(V_{i k}+V_{l k}\right)+V_{i k}\left(z-H_{i k}\right)^{-1}\left(V_{l k}+V_{i l}\right)\right] \\
& \times\left(z-H_{i l k}\right)^{-1} P_{i l k} \rho_{i l k} .
\end{aligned}
$$

Proof. Consider only $K_{1}(z)$; proofs for the others are similar. The extra assumption is required for $K_{2}(z)$ and $K_{3}(z)$ because they contain $P_{i l k}$ rather than $P_{i l j k}$. Hypotheses (1)-(3), and (5) imply $E_{i l, j k}<0$; Hypotheses (3) and (4) require $E_{i l k}<0$.

Compactness for $K_{1}(z)$ follows from Proposition V.15 for large negative $\operatorname{Re} z$, since $W_{i l}\left(z-H_{i l}\right)^{-1} V_{j k}\left(z-H_{i l, j k}\right)^{-1} P_{i l, j k} \rho_{i l, j k}$ is bounded for large negative $\operatorname{Re} z$ : 


$$
\begin{aligned}
K_{1}(z)= & W_{i j}\left(1-P_{i j}\right)\left(z-H_{i j}\right)^{-1}\left(V_{i k}+V_{j k}\right)\left(1-P_{i j k}\right)\left(z-H_{i j k}\right)^{-1} \\
& \times V_{i l}\left(z-H_{i l j k}\right)^{-1} P_{i l j k} \rho_{i l j k} \\
& -W_{i j}\left(1-P_{i j}\right)\left(z-H_{i j}\right)^{-1}\left(V_{i k}+V_{j k}\right)\left(1-P_{i j k}\right)\left(z-H_{i j k}\right)^{-1} \\
& \times V_{i l}\left(z-H_{i l}\right)^{-1} P_{i l j k} \rho_{i l j k} .
\end{aligned}
$$

By Proposition V.17, the second term is well behaved. The first term equals

$$
\begin{aligned}
W_{i j}\left(1-P_{i j}\right)(z & \left.-H_{i j}\right)^{-1}\left(V_{i k}+V_{j k}\right)\left(1-P_{i j k}\right)\left(z-H_{j k}\right)^{-1} \\
& \times V_{i l}\left(z-H_{i l j k}\right)^{-1} P_{i l j k} \rho_{i l j k} \\
& +W_{i j}\left(1-P_{i j}\right)\left(z-H_{i j}\right)^{-1}\left(V_{i k}+V_{j k}\right)\left(1-P_{i j k}\right)\left(z-H_{i j k}\right)^{-1} \\
& \times\left(V_{i j}+V_{i k}\right)\left(z-H_{j k}\right)^{-1} V_{i l}\left(z-H_{i l j k}\right)^{-1} P_{i l j k} \rho_{i l j k} \\
= & W_{i j}\left(1-P_{i j}\right)\left(z-H_{i j}\right)^{-1}\left(V_{i k}+V_{j k}\right)\left(1-P_{i j k}\right)\left(z-H_{i l j k}\right)^{-1} P_{i l j k} \rho_{i l j k} \\
& -W_{i j}\left(1-P_{i j}\right)\left(z-H_{i j}\right)^{-1}\left(V_{i k}+V_{j k}\right)\left(1-P_{i j k}\right)\left(z-H_{j k}\right)^{-1} P_{i l j k} \rho_{i l j k} \\
& +W_{i j}\left(1-P_{i j}\right)\left(z-H_{i j}\right)^{-1}\left(V_{i k}+V_{j k}\right)\left(1-P_{i j k}\right)\left(z-H_{i j k}\right)^{-1} \\
& \times\left(V_{i j}+V_{i k}\right)\left(z-H_{i l j k}\right)^{-1} P_{i l j k} \rho_{i l j k} \\
& -W_{i j}\left(1-P_{i j}\right)\left(z-H_{i j}\right)^{-1}\left(V_{i k}+V_{j k}\right)\left(1-P_{i j k}\right)\left(z-H_{i j k}\right)^{-1} \\
& \times\left(V_{i j}+V_{i k}\right)\left(z-H_{j k}\right)^{-1} P_{i l j k} \rho_{i l j k} .
\end{aligned}
$$

This may be written as a sum of seven terms!

$$
\begin{aligned}
\mathrm{I}(z)= & W_{i j}\left(1-P_{i j}\right)\left(z-H_{i j}\right)^{-1} V_{i k}\left(z-H_{i l j k}\right)^{-1} P_{i l j k} \rho_{i l j k}, \\
\mathrm{II}(z)= & W_{i j}\left(1-P_{i j}\right)\left(z-H_{i j}\right)^{-1} V_{j k}\left(z-H_{i l j k}\right)^{-1} P_{i l j k} \rho_{i l j k}, \\
\mathrm{III}(z)= & -W_{i j}\left(1-P_{i j}\right)\left(z-H_{i j}\right)^{-1}\left(V_{i k}+V_{j k}\right) P_{i j k}\left(z-H_{i l j k}\right)^{-1} P_{i l j k} \rho_{i l j k}, \\
\mathrm{IV}(z)= & -W_{i j}\left(1-P_{i j}\right)\left(z-H_{i j}\right)^{-1}\left(V_{i k}+V_{j k}\right)\left(1-P_{i j k}\right)\left(z-H_{j k}\right)^{-1} P_{i l j k} \rho_{i l j k}, \\
\mathrm{~V}(z)= & W_{i j}\left(1-P_{i j}\right)\left(z-H_{i j}\right)^{-1}\left(V_{i k}+V_{j k}\right)\left(1-P_{i j k}\right)\left(z-H_{i j k}\right)^{-1} \\
& \times\left(V_{i j}+V_{i k}\right)\left(z-H_{i l j k}\right)^{-1} P_{i l j k} \rho_{i l j k}, \\
\mathrm{VI}(z)= & -W_{i j}\left(1-P_{i j}\right)\left(z-H_{i j}\right)^{-1}\left(V_{i k}+V_{j k}\right)\left(1-P_{i j k}\right)\left(z-H_{i j k}\right)^{-1} P_{i l j k} \rho_{i l j k}, \\
\mathrm{VII}(z)= & W_{i j}\left(1-P_{i j}\right)\left(z-H_{i j}\right)^{-1}\left(V_{i k}+V_{j k}\right)\left(1-P_{i j k}\right)\left(z-H_{j k}\right) P_{i l j k} \rho_{i l j k} . \\
\mathrm{I}(z)= & \left(W_{i j}\left(1-P_{i j}\right)\left(z-H_{i j}\right)^{-1} U_{i k}\right)\left(W_{i k} P_{i l j k} \rho_{i l j k}^{-1}\right)\left(\rho_{i l j k}\left(z-H_{i l j k}\right)^{-1} \rho_{i l, j k}\right) .
\end{aligned}
$$

Apply Lemmas V.4, V.8, and II.3:

$$
\begin{aligned}
\mathrm{II}(z)= & W_{i j}\left(1-P_{i j}\right)\left(z-H_{0}\right)^{-1} V_{j k}\left(z-H_{i l j k}\right)^{-1} P_{i l j k} \rho_{i l, j k} \\
& +\left(W_{i j}\left(1-P_{i j}\right)\left(z-H_{i j}\right)^{-1} U_{i j}\right) \\
& \times\left(W_{i j}\left(z-H_{0}\right)^{-1} V_{j k}\left(z-H_{i l, j k}\right)^{-1} P_{i l j k} \rho_{i l j k}\right) .
\end{aligned}
$$


Lemma V.7 shows the first factor of the second term is well behaved.

$$
\left(z-H_{i l, j k}\right)^{-1} P_{i l, j k}=\left(z-E_{i l, j k}-K_{i l, j k}\right)^{-1} P_{i l, j k}
$$

and $\left(z-E_{i l, j k}-K_{i l, j k}\right)^{-1}$ commutes with $V_{j k}$. Moreover,

$$
\begin{aligned}
(z- & \left.H_{0}\right)^{-1}\left(z-E_{i l, j k}-K_{i l, j k}\right)^{-1} \\
& =\left[\left(z-H_{0}\right)^{-1}-\left(z-E_{i l, j k}-K_{i l j k}\right)^{-1}\right]\left(H_{0}^{\left(x_{i l}\right)}+H_{0}^{\left(x_{j k}\right)}-E_{i l j k}\right)^{-1} .
\end{aligned}
$$

The second factor of the second term in the expression for $\mathrm{II}(z)$ can now be written as:

$$
\begin{aligned}
\left(W _ { i j } \left[\left(z-H_{0}\right)^{-1}\right.\right. & \left.\left.-\left(z-E_{i l j k}-K_{i l j k}\right)^{-1}\right] \rho_{i l j k}\right) \\
& \times\left(\left(H_{0}^{\left(x_{i l}\right)}+H_{0}^{\left(x_{j k}\right)}-E_{i l j k}\right)^{-1} U_{j k}\right)\left(W_{j k} P_{i l j k}\right) .
\end{aligned}
$$

The methods of Lemma II.3 control the first factor; Corollary V.5 bounds the last factor; Theorem II.10 and Hypothesis (3) imply $E_{i l j k}$ is negative, so the middle factor is bounded (for $K_{2}$ and $K_{3}$ we need Hypothesis (4) at this point). The first term in the expression for $\operatorname{II}(z)$ is treated by the same method.

III(z) may be controlled by using Lemma V.4, the methods of Lemma II.3, and Corollary V.5.

$$
\begin{aligned}
\operatorname{IV}(z)= & W_{i j}\left(1-P_{i j}\right)\left(z-H_{i j}\right)^{-1}\left(V_{i k}+V_{j k}\right) P_{i j k}\left(z-H_{j k}\right)^{-1} P_{i l j k} \rho_{i l j k} \\
& -W_{i j}\left(1-P_{i j}\right)\left(z-H_{i j}\right)^{-1} V_{i k}\left(z-H_{j k}\right)^{-1} P_{i l j k} \rho_{i l j k} \\
& -W_{i j}\left(1-P_{i j}\right)\left(z-H_{i j}\right)^{-1} V_{j k}\left(z-H_{j k}\right)^{-1} P_{i l j k} \rho_{i l j k} .
\end{aligned}
$$

The first term may be controlled by the method used for III(z), above. Lemma V.12 controls the remaining terms.

If Proposition V.16 is replaced by Proposition V.15, then the argument for III(z) in Proposition V.29 shows that V(z) is well behaved.

$\mathrm{VI}(z)$ is well behaved by the proof of Proposition V.15.

$\operatorname{VII}(z)$ is well behaved by Lemma V.12.

VI. Generic couplings. Hypotheses (1), (2), and (4) of $\S \mathrm{V}$ hold for a large class of potentials (by Theorem II.10). However, Hypothesis (3) fails for some elements of any reasonable vector space of potentials. In this section, we show that Hypotheses (1) and (2) imply Hypothesis (3) for all couplings in the complement of a closed set of measure zero.

For the two body problem, Hypothesis (3) trivially holds, because there are no nontrivial cluster decompositions.

When $N=3$, there are three nontrivial cluster decompositions, with cluster Hamiltonians $h_{i j}=h_{0}^{(i j)}+\lambda_{i j} V_{i j}$. Hypothesis (3) states that 0 is not an eigenvalue or resonance energy for $h_{i j}$. This means 1 is not an eigenvalue of $\lambda_{i j} W_{i j}\left(0-h_{0}\right)^{-1} U_{i j}$.

When $\lambda_{i j}=0, \lambda_{i j} W_{i j}\left(0-h_{0}\right)^{-1} U_{i j}$ is the zero operator. Moreover, $\lambda_{i j} W_{i j}(0-$ $\left.h_{0}\right)^{-1} U_{i j}$ is a compact operator valued analytic function of $\lambda_{i j}$. So, the analytic Fredholm theorem shows that Hypothesis (3) fails only when $\lambda_{i j}$ belongs to some discrete subset $\Lambda_{i j} \subseteq \mathbf{R}$. 
Since $\left\{\left(\lambda_{12}, \lambda_{13}, \lambda_{23}\right) \in \mathbf{R}^{3}\right.$ : some $\left.\lambda_{i j} \in \Lambda_{i j}\right\}$ is closed and has codimension 1 , the set of couplings where Hypothesis (3) can fail is closed and has Lebesgue measure zero in $\mathbf{R}^{3}$.

For $N=4$, the situation is more complicated because Hypothesis (3) imposes the above conditions on the two body subsystems, as well as conditions on the three body subsystems. For each cluster Hamiltonian

$$
h_{i j k}=h_{0}^{(i, j, k)}+\lambda_{i j} V_{i j}+\lambda_{i k} V_{i k}+\lambda_{j k} V_{j k}
$$

we construct $M\left(\lambda_{i j}, \lambda_{i k}, \lambda_{j k}, z\right)$, as in equation (IV.6). Hypothesis (3) requires that 1 not be a eigenvalue of $M\left(\lambda_{i j}, \lambda_{i k}, \lambda_{j k}, z\right)$, whenever $z$ is a threshold for $h_{i j k}$. $M\left(\lambda_{i j}, \lambda_{i k}, \lambda_{j k}, z\right)$ is analytic in the $\lambda$ variables, except when some $\lambda_{n l}$ belongs to $\Lambda_{n l}$. At this point, $M$ is discontinuous, and the argument used above for $N=3$ is not valid.

For the nonzero thresholds (which must be negative), we can modify the argument so that it is valid in the four body case:

Lemma VI.1. Suppose $N=4$ and Hypotheses (1) and (2) hold. Let $\Gamma_{1} \subseteq \mathbf{R}^{6}$ be the set of couplings $\left\{\lambda_{i j}\right\}$, such that Hypothesis (3) fails for $H\left(\left\{\lambda_{i j}\right\}\right)=H_{0}+\Sigma_{i<j} \lambda_{i j} V_{i j}$ at a negative energy threshold. Then the closure of $\Gamma_{1}$ has Lebesgue measure zero.

Proof. Since the two body subsystems have no negative thresholds, the conclusion of the lemma is a statement about the three body subsystems only.

If $h_{i j k}$ is a three body cluster Hamiltonian, then a negative energy threshold $E$ for $h_{i j k}$ depends only on one coupling constant, which we may assume is $\lambda_{i j}$. Fix $\lambda_{i j} \notin \Lambda_{i j}$. The operator $M\left(\lambda_{i j}, \lambda_{i k}, \lambda_{j k}, E\left(\lambda_{i j}\right) \pm \mathrm{i} 0\right)$ associated to $h_{i j k}$, has a compact square and is analytic for $\left(\lambda_{i k}, \lambda_{j k}\right) \in \Omega$, where

$$
\Omega=\left\{\left(\lambda_{i k}, \lambda_{j k}\right) \in \mathbf{R}^{2}: \lambda_{i k} \notin \Lambda_{i k}, \lambda_{j k} \notin \Lambda_{j k}\right\}
$$

$\Omega$ is a union of open squares, and on each square $\Delta$ there are two possibilities:

(i) $1 \notin \sigma\left(M\left(\lambda_{i j}, \lambda_{i k}, \lambda_{j k}, E\left(\lambda_{i j}\right) \pm i 0\right)\right)$ for some $\left(\lambda_{i k}, \lambda_{j k}\right) \in \Delta$.

(ii) $1 \in \sigma\left(M\left(\lambda_{i j}, \lambda_{i k}, \lambda_{j k}, E\left(\lambda_{i j}\right) \pm i 0\right)\right)$ for all $\left(\lambda_{i k}, \lambda_{j k}\right) \in \Delta$.

When the first possibility occurs, the analytic Fredholm theorem shows that $1 \in \sigma\left(M\left(\lambda_{i j}, \lambda_{i k}, \lambda_{j k}, E\left(\lambda_{i j}\right) \pm i 0\right)\right)$ only for those $\left(\lambda_{i k}, \lambda_{j k}\right) \in \Delta$, which belong to a measure zero union of analytic varieties. The union of the boundary of $\Delta$ and these varieties is closed in $\mathbf{R}^{2}$.

Since $h_{i j k}$ has finitely many thresholds (see Simon [29]), we see that the intersection of $\Gamma_{1}$ with any compact set is contained in a finite union of closed sets of measure zero, if possibility (ii) above, never occurs. Thus, it suffices to prove that there is no square $\Delta$ such that $M\left(\lambda_{i j}, \lambda_{i k}, \lambda_{j k}, E\left(\lambda_{i j}\right) \pm i 0\right)$ has eigenvalue 1 for all $\left(\lambda_{i k}, \lambda_{j k}\right) \in \Delta$.

$M\left(\lambda_{i j}, 0,0, E\left(\lambda_{i j}\right) \pm i 0\right)=0$. So, if such a square $\Delta$ exists, we may assume (by changing squares if necessary) that $\Delta$ is adjacent to a square $\Delta_{1}$, on which possibility (i) occurs. Moreover, without loss, we may assume that it is possible to move from $\Delta_{1}$ to $\Delta$ by increasing $\lambda_{i k}$.

Fix $\lambda_{j k}$ so that $\left(\lambda_{i k}, \lambda_{j k}\right) \in \Delta_{1}$ for some $\lambda_{i k}$, and let $N\left(\lambda_{i k}, z\right)=M\left(\lambda_{i j}, \lambda_{i k}, \lambda_{j k}, z\right)$, where $\lambda_{i k}$ is chosen so that $\left(\lambda_{i k}, \lambda_{j k}\right) \in \Delta_{1}$. As we increase $\lambda_{i k}, N\left(\lambda_{i k}, z\right)$ is analytic 
until $\lambda_{i k}$ approaches $\omega \in \Lambda_{i k}$. At this point, $P_{i k}\left(\lambda_{i k}\right)$ has a discontinuity. However, by representing $P_{i k}\left(\lambda_{i k}\right)$ as a contour integral, we see that it has an analytic continuation $P_{i k}^{\prime}\left(\lambda_{i k}\right)$ from the region $\lambda_{i k} \in\left(\omega-\varepsilon_{1}, \omega\right)$ into the region $\lambda_{i k} \in(\omega-$ $\left.\varepsilon_{1}, \omega+\varepsilon_{2}\right)$, where $\varepsilon_{1}>0, \varepsilon_{2}>0$.

For $0<\lambda_{i k}-\omega<\varepsilon_{2}, P_{i k}\left(\lambda_{i k}\right)-P_{i k}^{\prime}\left(\lambda_{i k}\right)$ is the projection onto the new eigenfunctions of $h_{i k}\left(\lambda_{i k}\right) \otimes 1$, which appear as $\lambda_{i k}$ passes through $\omega$. Let $\beta\left(\lambda_{i k}\right)$ be the infimum of the corresponding new thresholds for $h_{i j k}$.

Replacing $P_{i k}\left(\lambda_{i k}\right)$ by $P_{i k}^{\prime}\left(\lambda_{i k}\right)$ in Lemmas V.4, V.8, and V.11-V.13, we see that $N\left(\lambda_{i k}, z\right)$ has an analytic continuation into the region $\lambda_{i k} \in\left(\omega-\varepsilon_{1}, \omega-\varepsilon_{2}\right)$, as long as $z \notin\left[\beta\left(\lambda_{i k}\right), \infty\right)$. Since $E\left(\lambda_{i j}\right)<0$ and $\beta(\omega)=0, N\left(\lambda_{i k}, E\left(\lambda_{i j}\right) \pm i 0\right)$ is analytic for $\omega-\varepsilon_{1}<\lambda_{i k}<\omega+\varepsilon_{3}$, for some $\varepsilon_{3}>0$.

When $\omega<\lambda_{i k}<\omega+\varepsilon_{3},\left(\lambda_{i k}, \lambda_{j k}\right) \in \Delta$. So, there exists a vector

$$
\phi\left(\lambda_{i k}\right)=\left[\begin{array}{c}
\phi_{i j, 1}\left(\lambda_{i k}\right) \\
\phi_{i j, 2}\left(\lambda_{i k}\right) \\
\phi_{i k, 1}\left(\lambda_{i k}\right) \\
\phi_{i k, 2}\left(\lambda_{i k}\right) \\
\phi_{j k, 1}\left(\lambda_{i k}\right) \\
\phi_{j k, 2}\left(\lambda_{i k}\right)
\end{array}\right],
$$

such that $\phi\left(\lambda_{i k}\right)=M\left(\lambda_{i j}, \lambda_{i k}, \lambda_{j k}, E\left(\lambda_{i j}\right) \pm i 0\right) \phi\left(\lambda_{i k}\right)$, whenever $\lambda_{i k}$ belongs to some open subset of $\left(\omega, \omega+\varepsilon_{3}\right)$. Define

$$
\psi\left(\lambda_{i k}\right)=\left[\begin{array}{l}
\phi_{i j, 1}\left(\lambda_{i k}\right) \\
\phi_{i j, 2}\left(\lambda_{i k}\right) \\
{\left[\phi_{i k, 1}\left(\lambda_{i k}\right)+\lambda_{i k} W_{i k}\left(P_{i k}\left(\lambda_{i k}\right)\right.\right.} \\
\left.\left.-P_{i k}^{\prime}\left(\lambda_{i k}\right)\right) G_{i k}\left(\lambda_{i k}\right) \rho_{i k} \phi_{i k, 2}\left(\lambda_{i k}\right)\right] \\
P_{i k}^{\prime}\left(\lambda_{i k}\right) \phi_{i k, 2}\left(\lambda_{i k}\right) \\
\phi_{j k, 1}\left(\lambda_{i k}\right) \\
\phi_{j k, 2}\left(\lambda_{i k}\right)
\end{array}\right]
$$

Then $\psi\left(\lambda_{i k}\right)=N\left(\lambda_{i k}, E\left(\lambda_{i j}\right) \pm i 0\right) \psi_{i k}\left(\lambda_{i k}\right)$ for all $\lambda_{i k}$ in the same open subset of $\left(\omega, \omega+\varepsilon_{3}\right)$.

The analytic Fredholm theorem now shows that $1 \in \sigma\left(N\left(\lambda_{i k}, E\left(\lambda_{i j}\right) \pm i 0\right)\right)$ for $\omega-\varepsilon_{1}<\lambda_{i k}<\omega$. Since this is true for all choices of $\lambda_{j k}$, such that $\left(\lambda_{i k}, \lambda_{j k}\right) \in \Delta_{1}$ for some $\lambda_{i k}$, we conclude that $M\left(\lambda_{i j}, \lambda_{i k}, \lambda_{j k}, E\left(\lambda_{i j}\right) \pm i 0\right)$ has a constant eigenvalue 1 for all $\left(\lambda_{i k}, \lambda_{j k}\right) \in \Delta_{1}$. This contradicts the choice of $\Delta_{1}$, and the lemma is proved.

REMARK. The above argument fails for the zero energy threshold, because the new thresholds appear at zero when $\lambda_{i k}$ passes $\omega$. To avoid this problem, we move $\lambda_{i k}$ around $\omega$ in the complex plane. The next lemma shows that we may do so without having the cuts of $\sigma_{\text {ess }}\left(h_{i j k}\right)$ cross the negative real axis. 
LeMma VI.2. Assume Hypotheses (1) and (2) for the two body Hamiltonian $H(\lambda)=H_{0}+\lambda V$. There is an open complex neighborhood $X$ of the real axis, such that $\lambda \in X$ and $\operatorname{Im} \lambda \neq 0$ imply that $H(\lambda)$ has no eigenvalues in the real interval $(-\infty, 0]$. Moreover, $\lambda \in X$ and $\operatorname{Im} \lambda \neq 0$ imply $H(\lambda)$ has no zero energy resonances.

Proof. The analytic Fredholm theorem shows that $1 \in \sigma\left(\lambda W\left(0-H_{0}\right)^{-1} U\right)$ only when $\lambda$ belongs to a discrete subset $\Lambda$ of the complex plane. There exists an open neighborhood $X$ of the real axis, such that $\Lambda \cap X \subseteq \mathbf{R}$. Since $H(\lambda)$ has a zero energy resonance or eigenvalue if and only if $\lambda \in \Lambda$, we need only show that Im $\lambda \neq 0$ implies $H(\lambda)$ has no eigenvalues in $(-\infty, 0)$.

Suppose $H(\lambda) \phi=E \phi$, where $\operatorname{Im} \lambda \neq 0$ and $E<0$.

$$
\left\langle\phi, H_{0} \phi\right\rangle+\lambda\langle\phi, V \phi\rangle=E\langle\phi, \phi\rangle\langle 0 \text {. }
$$

Since $\left\langle\phi, H_{0} \phi\right\rangle \geqslant 0, \lambda\langle\phi, V \phi\rangle$ must be negative. Since $\langle\phi, V \phi\rangle \in \mathbf{R}, \operatorname{Im} \lambda$ must be zero. This contradicts $\operatorname{Im} \lambda \neq 0$.

Proposition VI.3. Assume Hypotheses (1) and (2) for the $N \leqslant 4$ body Hamiltonian

$$
H\left(\left\{\lambda_{i j}\right\}\right)=H_{0}+\sum_{i<j} \lambda_{i j} V_{i j}
$$

Let $\Gamma$ denote the set of all couplings $\left\{\lambda_{i j}\right\}$ such that Hypothesis (3) fails for $H\left(\left\{\lambda_{i j}\right\}\right)$. Then $\Gamma$ is closed and has Lebesgue measure zero.

Proof. $\Gamma$ is closed because eigenvalues and thresholds depend continuously on the couplings.

When $N=2$ or 3 , the discussion at the beginning of this section proves the proposition. When $N=4$, Lemma IV.1 shows that we need only study the threshold at zero energy.

The method of proof used for Lemma VI.1 may be applied to the zero energy threshold, with the following change. We cannot analytically continue $N\left(\lambda_{i k}, 0 \pm\right.$ $i 0)$ through the point $\omega \in \Lambda_{i k}$. However, Lemmas V.4, V.8, and V.11-V.13, and VI.2 show that we may continue $N\left(\lambda_{i k}, 0 \pm i 0\right)$ from the region $\omega-\varepsilon_{1}<\lambda_{i k}<\omega$ $\left(\lambda_{i k} \in \mathbf{R}\right)$ into the region

$$
R=\left\{\lambda_{i k}=\omega+r e^{i \theta}: 0<r<\varepsilon, 0<\arg \theta<2 \pi\right\} .
$$

These lemmas also show that $M\left(\lambda_{i j}, \lambda_{i k}, \lambda_{j k}, 0+i 0\right)$ and $M\left(\lambda_{i j}, \lambda_{i k}, \lambda_{j k}, 0-i 0\right)$ have analytic continuations from the region $\omega<\lambda_{i k}<\omega+\delta$ into regions which intersect $R$ in open subsets of the complex plane.

Using the fact that the analytically continued $M$ operators have constant eigenvalue 1 , we conclude that the analytically continued $N$ operators have constant eigenvalue 1. (This is done as in Lemma VI.1, by explicitly constructing the eigenfunction of $N$ from the eigenfunction of $M$, for $\lambda_{i k}$ in the open set to which both operators have been continued.)

This argument shows that $M\left(\lambda_{i j}, \lambda_{i k}, \lambda_{j k}, 0 \pm i 0\right)$ has constant eigenvalue 1 on the square $\Delta_{1}$, as in the proof of Lemma VI.1. This contradicts the choice of $\Delta_{1}$ and proves the proposition. 
REMARK. The coupling constants $\lambda_{i j}$ and $\lambda_{i k}$ are treated very differently in the proof of Lemma VI.1. So, our results say nothing about the case in which various coupling constants must be equal.

Appendix. Representative sample of the blocks of the matrices $A(z), C(z)$, and $M(z)$ discussed in \$IV.

\section{The Matrix $A(z)$}

$$
\begin{aligned}
& A_{i j}(z) \quad=\left[\begin{array}{llll}
G_{0} u_{i j} & G_{0} v_{i j} G_{i j} P_{i j} \rho_{i j} & 0
\end{array}\right] \\
& A_{i j, i j k}(z)=\left[\begin{array}{lll}
G_{0} v_{i j} & G_{0} v_{i j} G_{i j} P_{i j} \rho_{i j, k} & \frac{1}{3} G_{0}\left[v_{i j} G_{i j}\left(v_{i k}+v_{j k}\right)+v_{i k} G_{i k}\left(v_{i j}+v_{j k}\right)\right. \\
& \left.+v_{j k} G_{j k}\left(v_{i j}+v_{i k}\right)\right] G_{i j k} P_{i j k} \rho_{i j k}
\end{array}\right] \\
& A_{i j,(i j, k \ell)}(z)=\left[\begin{array}{lll}
G_{0} U_{i j} & G_{0} v_{i j} G_{i j} P_{i j} U_{k \ell} & \\
& & \frac{l}{2} G_{0}\left[v_{i j} G_{i j} v_{k \ell}+v_{k \ell} G_{k \ell} v_{i j}\right] \\
& \times G_{i j, k \ell} P_{i j, k \ell}{ }^{P_{i j}, k \ell}
\end{array}\right]
\end{aligned}
$$

\section{The Matrix C(z)}

$$
\begin{aligned}
& C_{i j}(z) \quad=\left[\begin{array}{l}
W_{i j} G_{i j}\left(1-P_{i j}\right) \\
\rho_{i j}^{-1} P_{i j} \\
0
\end{array}\right] \\
& c_{i j, i j k}(z)=\left[\begin{array}{l}
w_{i j} G_{i j}\left(1-p_{i j}\right)\left(v_{i k}+v_{i k}\right) G_{i j k}\left(1-p_{i j k}\right) \\
\rho_{i j, k}^{-1} P_{i j}\left(v_{i k}+v_{j k}\right) G_{i j k}\left(1-p_{i j k}\right) \\
\rho_{i j k}^{-1} p_{i j k}
\end{array}\right] \\
& c_{i j,(i j, k \ell)}(z)=\left[\begin{array}{l}
w_{i j} G_{i j}\left(1-P_{i j}\right) v_{k \ell} G_{i j, k \ell}\left(1-P_{i j, k \ell}\right) \\
P_{i j} W_{k \ell} G_{i j, k \ell}\left(1-P_{i j, k \ell}\right) \\
\rho_{i j, k \ell}-1
\end{array}\right]
\end{aligned}
$$


12,$123 ; 14,124$

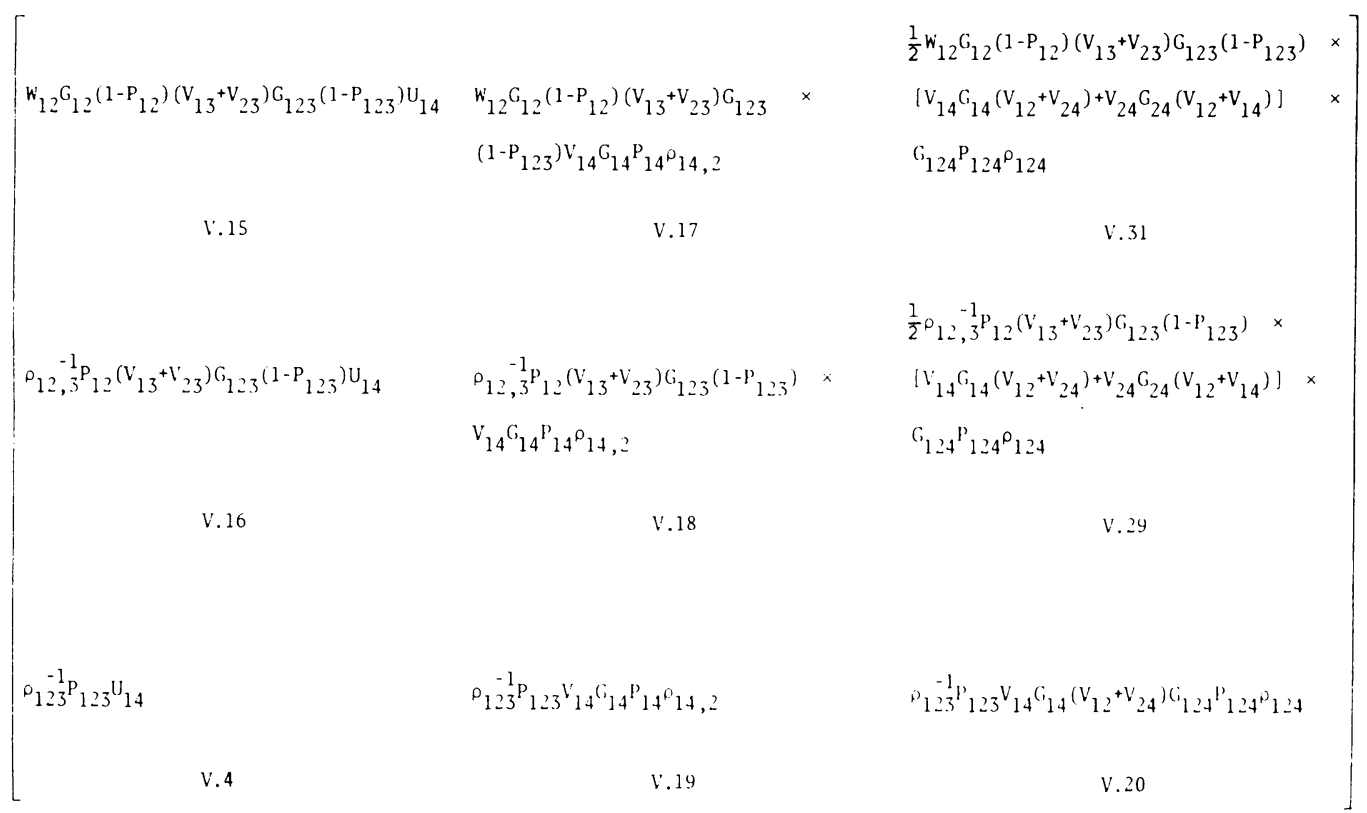

\section{2,$123 ; 34,134$}

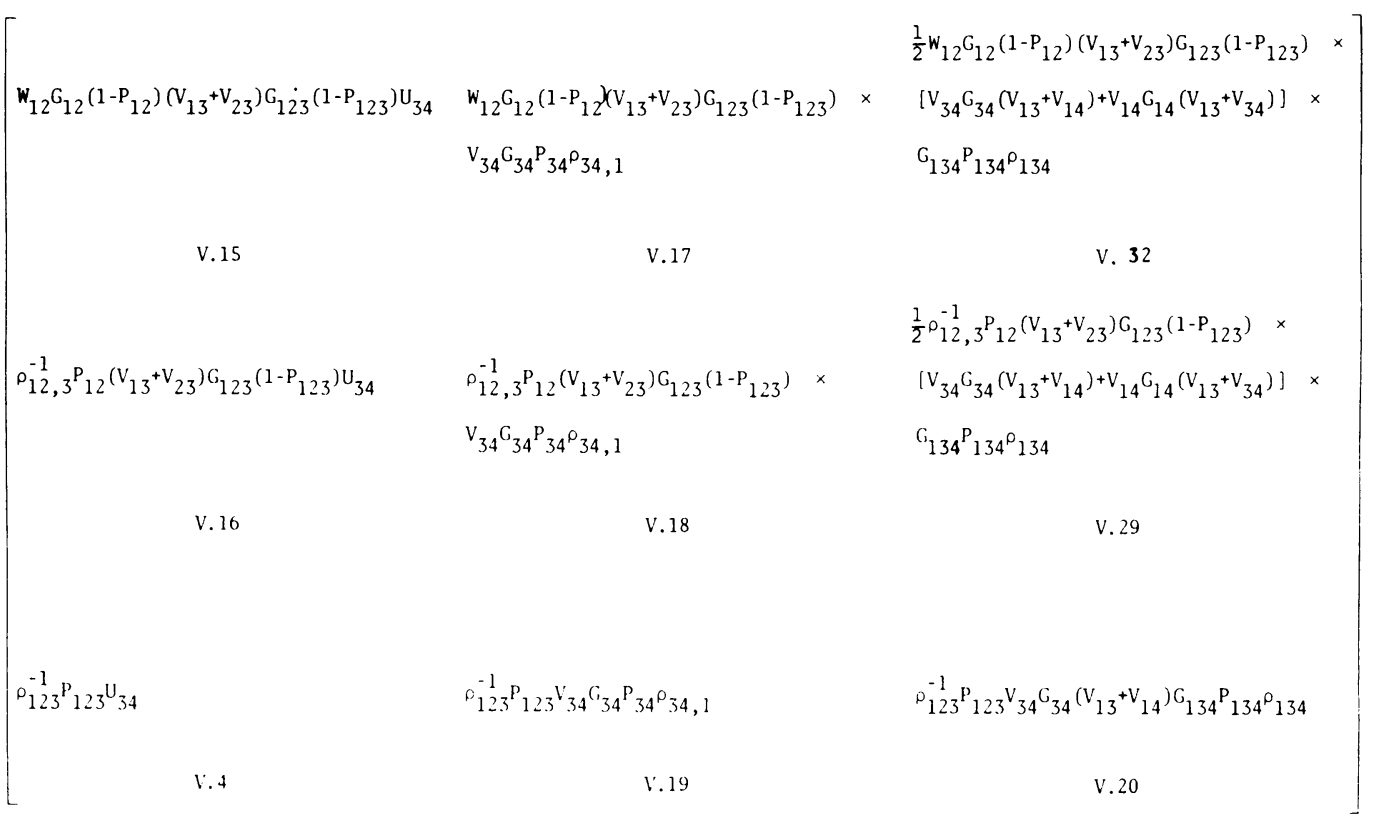




\section{G. A. HAGEDORN}

\section{2,$123 ; 14,134$}

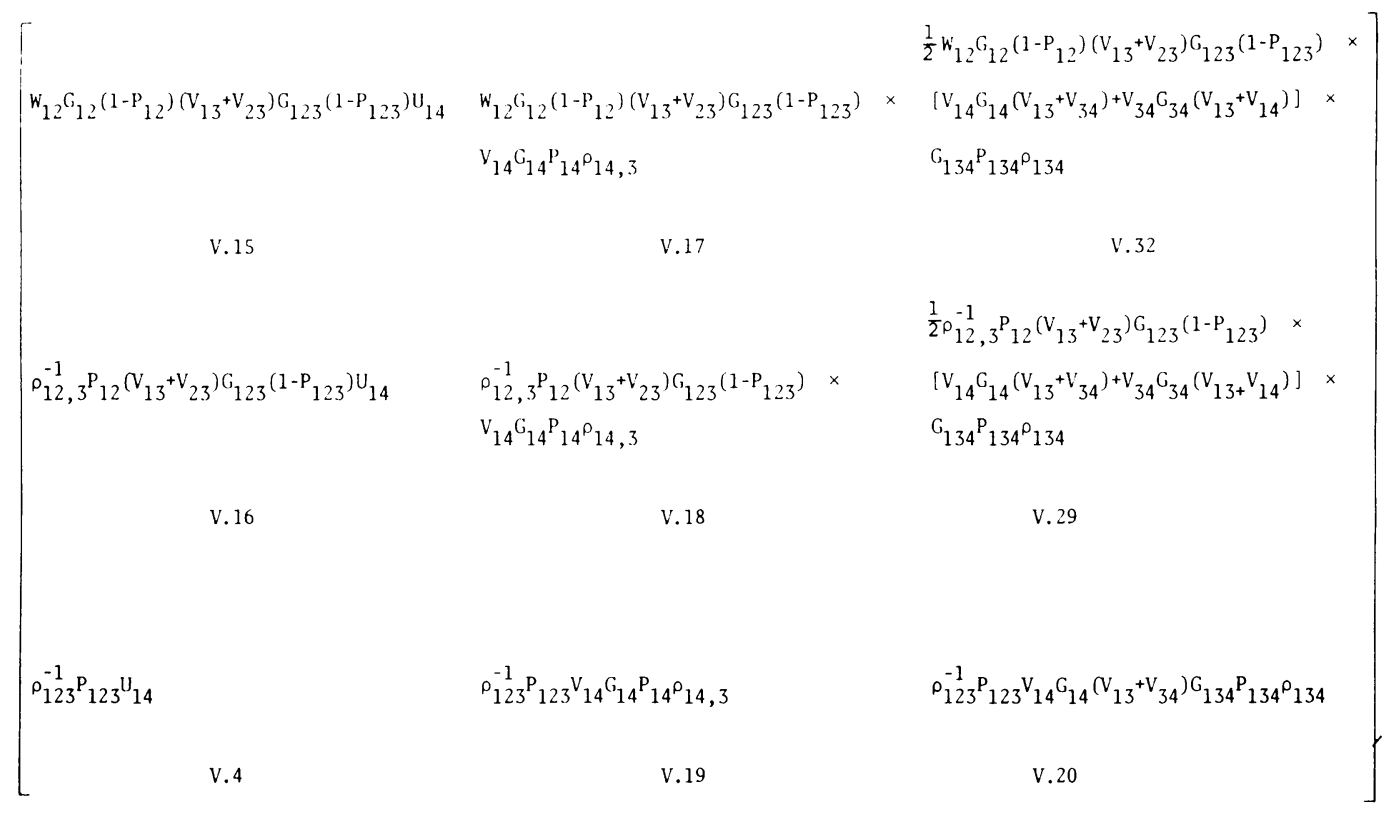

\section{$12,(12,34) ; 13,(13,24)$}

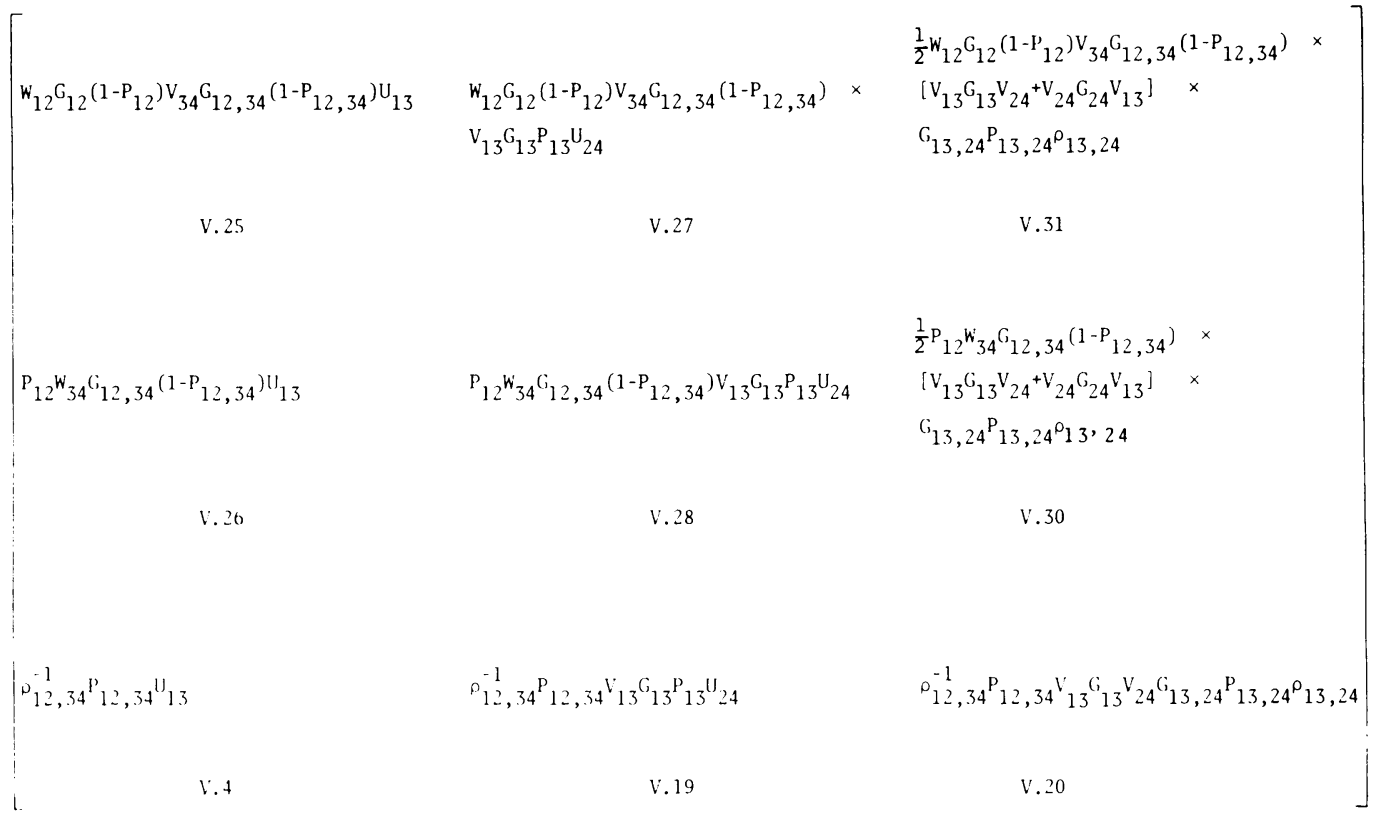




\begin{tabular}{|c|c|c|}
\hline $\mathrm{w}_{12} \mathrm{(i}_{12}\left(1-\mathrm{P}_{12}\right)\left(\mathrm{v}_{13}+\mathrm{l}_{23}\right) \mathrm{i}_{123}\left(1-\mathrm{P}_{123}\right) \mathrm{u}_{14}$ & $\mathrm{w}_{12}\left(\mathrm{i}_{12}\left(1-\mathrm{P}_{12}\right)\left(\mathrm{v}_{13}+\mathrm{v}_{23}\right) \mathrm{i}_{123}\left(1-\mathrm{P}_{123}\right)\right.$ & $\mathrm{K}_{12} \mathrm{C}_{12}\left(1-\mathrm{P}_{12}\right)\left(\mathrm{V}_{13}+\mathrm{l}_{23}\right) \mathrm{G}_{123}\left(1-\mathrm{P}_{123}\right) \times$ \\
\hline & $i_{14}\left(i_{14} P_{14} U_{23}\right.$ & 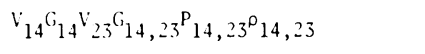 \\
\hline 1.15 & 1.17 & 1.32 \\
\hline$o_{12,3}^{-1} \mathrm{P}_{12}\left(\mathrm{~V}_{13}+\mathrm{v}_{23}\right) \mathrm{i}_{123}\left(1-\mathrm{P}_{123}\right) \mathrm{U}_{14}$ & $\rho_{12,3}^{-1} \mathrm{P}_{12}\left(\mathrm{~V}_{13}+\mathrm{l}_{23}\right)\left(\mathrm{i}_{123}\left(1-\mathrm{P}_{123}\right) \times\right.$ & $0_{12,3}^{-1} \mathrm{P}_{12}\left(\left(_{13}+l_{23}\right)\left(\mathrm{c}_{123}\left(1-\mathrm{P}_{123}\right) \times\right.\right.$ \\
\hline & $V_{14}{ }^{C_{1}} 14^{P_{1}} 4_{23} U_{23}$ & 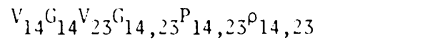 \\
\hline V.16 & 1.18 & 1.29 \\
\hline$\rho_{123}^{-1} \mathrm{P}_{123} \mathrm{U}_{14}$ & $\rho_{123}^{-1} P_{123} V_{14} C_{14} P_{14} U_{23}$ & $\rho_{123}^{-1} P_{12} 3_{14}^{V^{C}} C_{14} V_{23}{ }^{C_{1}} 14,23^{P} 14,23^{\rho_{14}}, 23$ \\
\hline V. 4 & V. 19 & V. 20 \\
\hline
\end{tabular}

\section{2,$123 ; 34,(12,34)$}

\begin{tabular}{|c|c|c|}
\hline $\mathrm{W}_{12} \mathrm{G}_{12}\left(1-\mathrm{P}_{12}\right)\left(\mathrm{V}_{13}+\mathrm{V}_{23}\right) \mathrm{G}_{123}\left(1-\mathrm{P}_{123}\right) \mathrm{U}_{34}$ & $\begin{array}{l}\mathrm{W}_{12} \mathrm{G}_{12}\left(1-\mathrm{P}_{12}\right)\left(\mathrm{V}_{13}+\mathrm{V}_{23}\right) \mathrm{G}_{123}\left(1-\mathrm{P}_{123}\right) \times \\
\mathrm{V}_{34} \mathrm{G}_{34} \mathrm{P}_{34} \mathrm{U}_{12}\end{array}$ & $\begin{array}{l}W_{12} G_{12}\left(1-P_{12}\right)\left(V_{13}+V_{23}\right) G_{123}\left(1-P_{123}\right) \times \\
V_{34} G_{34} V_{12} G_{12}, 34{ }_{12,34} P_{12,34}\end{array}$ \\
\hline V.15 & V.17 & V.31 \\
\hline$\rho_{12,3}^{-1} \mathrm{P}_{12}\left(\mathrm{~V}_{13}+\mathrm{V}_{23}\right) \mathrm{C}_{123}\left(1-\mathrm{P}_{123}\right) \mathrm{U}_{34}$ & $\rho_{12,3}^{-1} \mathrm{P}_{12}\left(\mathrm{~V}_{13}+\mathrm{V}_{23}\right) \mathrm{G}_{123}\left(1-\mathrm{P}_{123}\right) \times$ & $\mathrm{o}_{12,3}^{-1} \mathrm{P}_{12}\left(\mathrm{~V}_{13}+\mathrm{V}_{23}\right) \mathrm{G}_{123}\left(1-\mathrm{P}_{123}\right) \times$ \\
\hline & $\mathrm{V}_{34} \mathrm{C}_{34} \mathrm{P}_{34} \mathrm{U}_{12}$ & $V_{34}{ }^{C_{34}}{ }_{12}{ }^{C_{12}} 12,34^{P}{ }_{12,34}{ }^{P} 12,34$ \\
\hline 1.10 & $v .18$ & 1.29 \\
\hline$\rho_{123}^{-1} \mathrm{P}_{123_{34}}^{\mathrm{U}_{3}}$ & 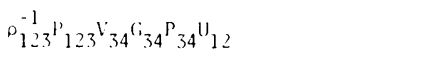 & $\mathrm{O}_{123}^{-1} \mathrm{P}_{123} \mathrm{~V}_{34} \mathrm{C}_{34} \mathrm{~V}_{12} \mathrm{G}_{12,34} \mathrm{P}_{12,34} \mathrm{\rho}_{12,34}$ \\
\hline 1.4 & V.19 & 1.20 \\
\hline
\end{tabular}




\section{$12,(12,34) ; 13,123$}

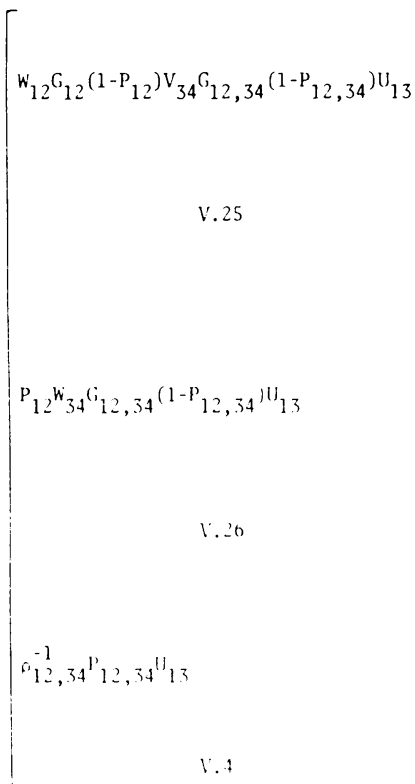

$\mathrm{W}_{12} \mathrm{C}_{12}\left(1-\mathrm{P}_{12}\right) \mathrm{V}_{34} \mathrm{C}_{12,34}\left(1-\mathrm{P}_{12,34}\right) \quad \times$ $\mathrm{V}_{13} \mathrm{G}_{13} \mathrm{P}_{13^{\mathrm{P}} 13,2}$

$$
\begin{aligned}
& \frac{1}{2} W_{12} C_{12}\left(1-P_{12}\right) V_{34} C_{12,34}\left(1-P_{12,34}\right) \times \\
& {\left[V_{13} G_{13}\left(V_{12}+V_{23}\right)+V_{23} G_{23}\left(V_{12}+V_{13}\right)\right] \times} \\
& { }_{{ }_{1}}{ }_{12} P_{123}{ }^{D_{123}}
\end{aligned}
$$

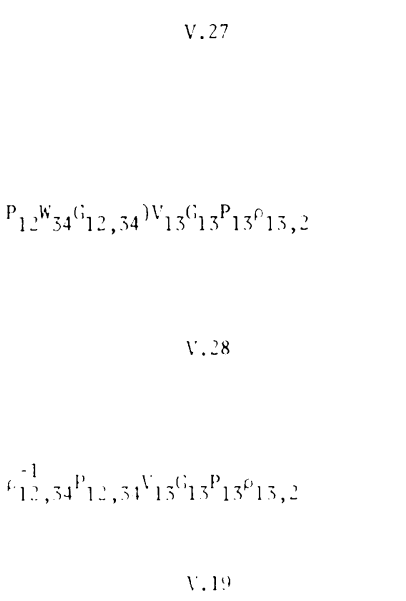

$$
\begin{aligned}
& \frac{1}{2} \mathrm{P}_{12} \mathrm{~W}_{34}{ }^{\mathrm{C}_{1}} 12,34\left(1-\mathrm{P}_{12,34}\right) \times
\end{aligned}
$$

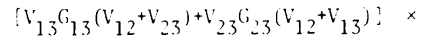

$$
\begin{aligned}
& { }^{\mathrm{G}_{123}} \mathrm{P}_{123^{\mathrm{P}}}{ }_{123} \\
& \text { 1. } 30
\end{aligned}
$$

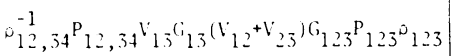

$$
\begin{aligned}
& \text { 1. } 20
\end{aligned}
$$

\section{$12,(12,34) ; 13,134$}

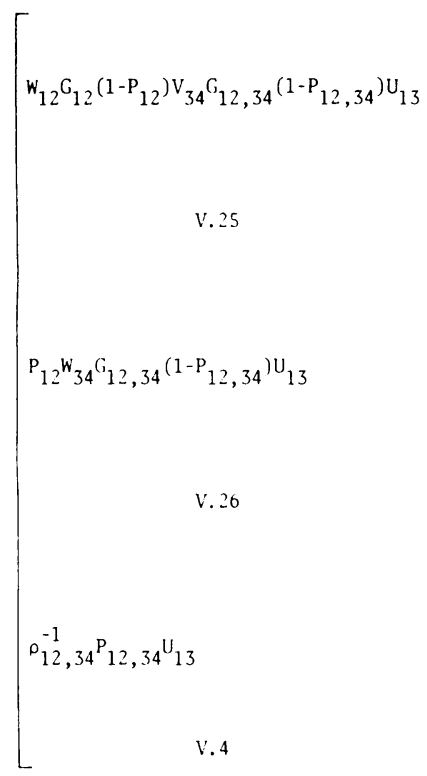

$$
\begin{aligned}
& \frac{1}{2} W_{12}{ }^{C_{12}}\left(1-P_{12}\right) V_{34} C_{12,34}\left(1-P_{12,34}\right) \times \\
& {\left[V_{13} G_{13}\left(V_{14}^{\prime}+V_{34}\right)+V_{34} G_{34}\left(V_{14}+V_{13}\right)\right] \times} \\
& \mathrm{G}_{134} \mathrm{P}_{134} \mathrm{\rho}_{134} \\
& \text { v. } 32 \\
& \frac{1}{2} P_{12}{ }^{W_{34}}{ }^{(i} 12,34\left(1-P_{12}, 34\right) \times \\
& v_{13}\left(i_{13}\left(v_{14}+v_{34}\right)+v_{34}\left(i_{3.4}\left(v_{14}+v_{13}\right)\right) \times\right. \\
& { }^{(i} 1.34^{\mathrm{P}} 134^{\mathrm{o}} 1.34 \\
& \text { 1. } 30
\end{aligned}
$$

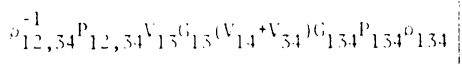

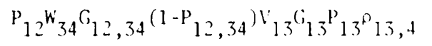$$
0_{12,34}^{-1} \mathrm{P}_{12}^{\mathrm{P}}, 3.4_{13}^{\mathrm{V}_{1}} 13^{\mathrm{P}} 13^{\mathrm{P}} 13,4
$$ 


\section{2,$123 ; 14$}

\begin{tabular}{|c|c|c|}
\hline $\mathrm{w}_{12} \mathrm{G}_{12}\left(1-\mathrm{P}_{12}\right)\left(\mathrm{V}_{13}+\mathrm{V}_{23}\right) \mathrm{G}_{123}\left(1-\mathrm{P}_{123}\right) \mathrm{U}_{14}$ & $W_{12} G_{12}\left(1-P_{12}\right)\left(V_{13}+V_{23}\right)\left(i_{123}\left(1-P_{123}\right) V_{14} C_{14} P_{14}{ }^{G} 14\right.$ & 0 \\
\hline V. 15 & v.17 & \\
\hline$\rho_{12,3}^{-1} P_{12}\left(V_{13}+V_{23}\right) G_{123}\left(1-P_{123}\right) U_{14}$ & $\rho_{12,3}^{-1}{ }^{P_{12}}\left(V_{13}+V_{23}\right) G_{123}\left(1-P_{123}\right) V_{14}{ }^{\left({ }_{1}\right.}{ }_{14} P_{14}{ }^{\rho} 14$ & 0 \\
\hline V.16 & V.18 & \\
\hline$\rho_{123}^{-1} P_{123}{ }_{14}$ & 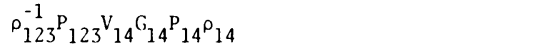 & 0 \\
\hline V. 4 & V.19 & \\
\hline
\end{tabular}

\section{2,$123 ; 34$}

$$
\begin{aligned}
& W_{12} G_{12}\left(1-P_{12}\right)\left(V_{13}+V_{23}\right) G_{123}\left(1-P_{123}\right) U_{34} \quad W_{12} G_{12}\left(1-P_{12}\right)\left(V_{13}+V_{23}\right) G_{123}\left(1-P_{123}\right) V_{34} G_{34} P_{34} \rho_{34} \\
& \text { V. } 17 \\
& \text { V. } 15 \\
& \rho_{12,3}^{-1} \mathrm{P}_{12}\left(\mathrm{~V}_{13}+\mathrm{V}_{23}\right) \mathrm{G}_{123}\left(1-\mathrm{P}_{123}\right) \mathrm{U}_{34} \\
& \rho_{12,3}^{-1} \mathrm{P}_{12}\left(\mathrm{~V}_{13}+\mathrm{V}_{23}\right) \mathrm{G}_{123}\left(1-\mathrm{P}_{123}\right) \mathrm{V}_{34}{ }^{\left(\mathrm{i}_{34} \mathrm{P}_{34} \mathrm{P}_{34}\right.} \\
& \text { V. } 16 \\
& \text { V. } 18 \\
& \rho_{123}^{-1} P_{123} \mathrm{U}_{34}
\end{aligned}
$$

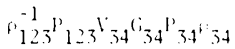




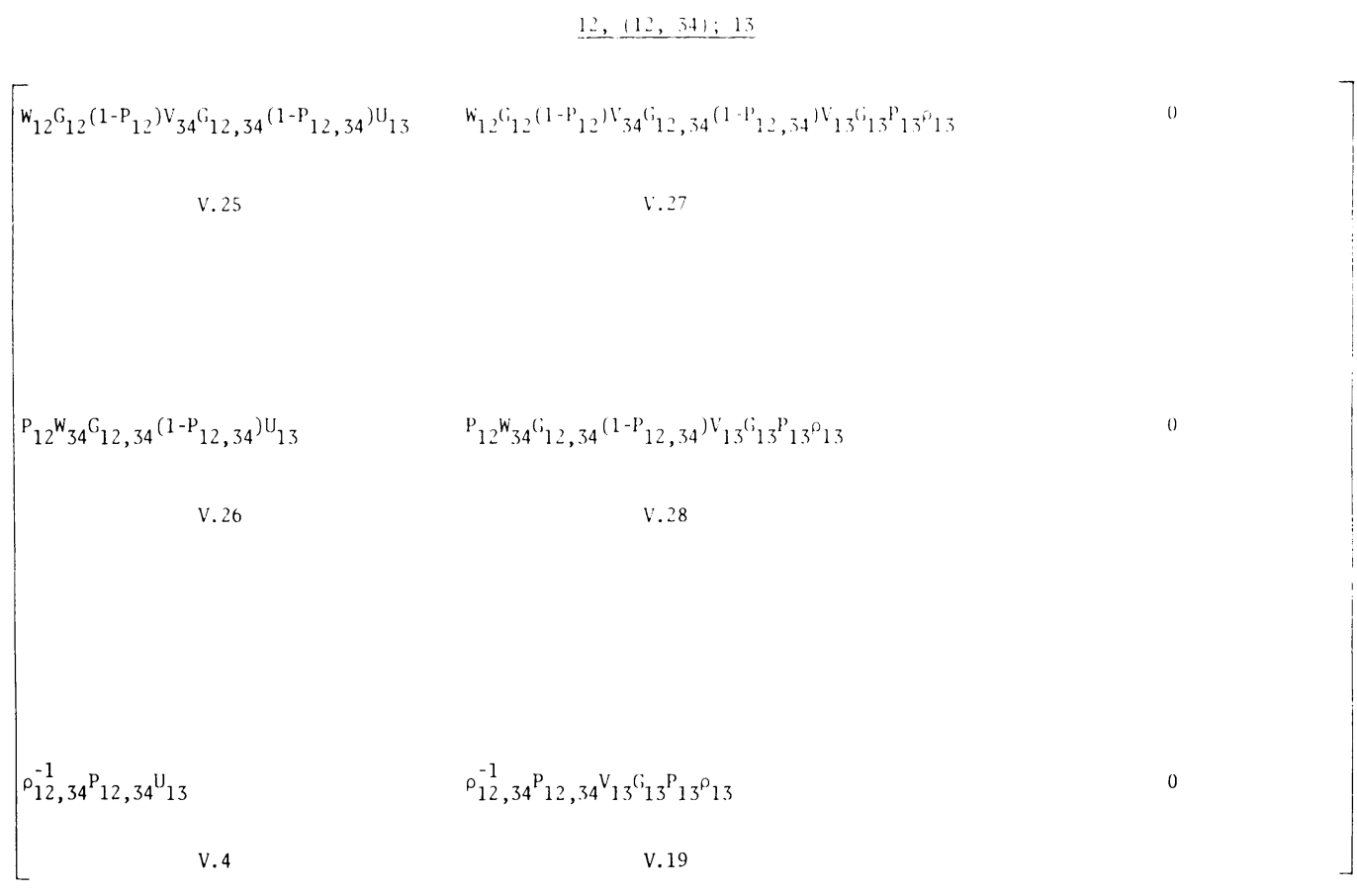

\section{REFERENCES}

1. S. Agmon, Spectral properties of Schrödinger operators and scattering theory, Ann. Scuola Norm. Sup. Pisa (IV) 2 (1975), 151-218.

2. E. Balslev, Absence of positive eigenvalues of Schrödinger operators, Arch. Rational Mech. Anal. 59 (1975), 343-357.

3. E. Balslev and J. M. Combes, Spectral properties of many-body Schrödinger operators with dilatation-analytic interactions, Comm. Math. Phys. 22 (1971), 280-294.

4. J. M. Combes and L. Thomas, Asymptotic behavior of Eigenfunctions for multiparticle Schrödinger operators, Comm. Math. Phys. 34 (1973), 251-270.

5. J. Cook, Convergence of the Moller wave matrix, J. Math. Phys. 36 (1957), 82-87.

6. L. D. Faddeev, Mathematical aspects of the three-body problem in the quantum scattering theory, Israel Program for Scientific Translations, Jerusalem, 1965.

7. J. Ginibre and M. Moulin, Hilbert space approach to the quantum mechanical three-body problem, Ann. Inst. H. Poincaré Sect. A 21 (1974), 97-145.

8. U. Greifenegger, K. Jörgens, J.Weidmann and M. Winkler, Streutheorie für Schrödinger Operatoren, preprint, 1972.

9. G. A. Hagedorn, Asymptotic completeness for a class of four particle Schrödinger operators, Bull. Amer. Math. Soc. 84 (1978), 155-156.

10. K. Hepp, On the quantum mechanical N-body problem, Helv. Phys. Acta 42 (1969), 425-458.

11. J. S. Howland, Abstract stationary theory of multichannel scattering, J. Functional Analysis 22 (1976), 250-282.

12. T. Ikebe, Eigenfunctions associated with the Schrödinger operators and their applications to scattering theory, Arch. Rational Mech..Anal. 5 (1960), 1-34.

13. R. J. Iorio and M. O'Carrol, Asymptotic completeness for multi-particle Schrödinger Hamiltonians with weak potentials, Comm. Math. Phys. 27 (1972), 137-145.

14. T. Kato, Perturbation theory for liner operators, Springer-Verlag, Berlin-Heidelberg-New York, 1966.

15. 258-279. Wave operators and similarity for some non-selfadjoint operators, Math. Ann. 162 (1966), 
16. S. T. Kuroda, An abstract stationary approach to perturbation of continuous spectra and scattering theory, J. Analyse Math. 20 (1967), 57-117.

17. __ Scattering theory for differential operators. II. Self-adjoint elliptic operators, J. Math. Soc. Japan 25 (1973), 222-234.

18. R. B. Lavine, Commutators and scattering theory. I. Repulsive interactions, Comm. Math. Phys. 20 (1971), 301-323.

19. E. Mourre, Applications de la méthode de Lavine au problème à trois corps, Ann. Inst. H. Poincaré Sect. A 26 (1977), 219-262.

20. I. M. Narodetskii and O. A. Yakubovskii, Two cluster approximation in the integral equation for $N$ particles, Soviet J. Nuclear Phys. 14 (1972), 178-184.

21. A. J. O'Connor, Exponential decay of bound state wave functions, Comm. Math. Phys. 32 (1973), 319-340.

22. M. Reed and B. Simon, Methods of modern mathematical physics, Vol. II: Fourier analysis, self-adjointness, Academic Press, New York-London, 1975.

23. __ Methods of modern mathematical physics, Vol. III: Scattering theory, Academic Press, New York-London, 1979.

24. __ Methods of modern mathematical physics, Vol. IV: Analysis of operators, Academic Press, New York-London, 1978.

25. M. Schechter, A new criterion for scattering theory, Preprint, Yeshiva University, 1976.

26. I. M. Sigal, Mathematical foundations of quantum scattering theory for multiparticle systems, Mem. Amer. Math. Soc. No. 20916 (1978).

27. Phys. 48 (1976), 155-164.

28. On quantum mechanics of many-body systems with dilation-analytic potentials, Bull. Amer. Math. Soc. 84 (1978), 152-154.

29. B. Simon, Quantum mechanics for Hamiltonians defined as quadratic forms, Princeton University Press, 1971.

30. __ Quadratic form techniques and the Balslev-Combes theorem, Comm. Math. Phys. 27 (1972), $1-9$.

31. __ Resonances in n-body quantum systems with dilation analytic potentials and the foundations of time-dependent perturbation theory, Ann. of Math. 97 (1973), 247-274.

32. __ Absence of positive eigenvalues in a class of multiparticle quantum systems, Math. Ann. 207 (1974), 133-138.

33. _ Scattering theory and quadratic forms: On a theorem of Schechter, Comm. Math. Phys. 53 (1977), 151-153.

34. 259-274.

35. E. M. Stein, Interpolation of linear operators, Trans. Amer. Math. Soc. 83 (1956), 482-492.

36. L. E. Thomas, Asymptotic completeness in two- and three-particle quantum mechanical scattering, Ann. Physics 90 (1975), 127-165.

37. E. C. Titchmarsh, Introduction to the theory of Fourier integrals, Oxford Press, London, 1950.

38. C. van Winter, Complex dynamical variables for multiparticle systems and analytic interactions. I, J. Math. Anal. Appl. 47 (1974), 633-670.

39. Complex dynamical variables for multiparticle systems with analytic interactions. II, J. Math. Anal. Appl. 48 (1974), 368-399.

40. S. Weinberg, Systematic solution of multiparticle scattering problems, Phys. Rev. B 133 (1964), $232-256$.

41. O. A. Yakubovskii, On the integral equations in the theory of $N$ particle scattering, Soviet J. Nuclear Phys. 5 (1967), 937-942.

42. V. Enss, Asymptotic completeness for quantum mechanical potential scattering, Comm. Math. Phys. (to appear).

43. C. van Winter, Projection operators associated with analytic multiparticle Hamiltonians, Notices Amer. Math. Soc. 25 (1978), A-320.

Department of Mathematics, Princeton University, Princeton, New Jersey 08540

Current address: Department of Mathematical Physics, The Rockefeller University, New York, New York 10021 